Supporting Information

Asymmetric Direct Vinylogous Conjugate Addition of Substituted Furanone Derivatives to Benzoyl Acrylonitrile: Stereoselective Synthesis Toward Bicyclic $\gamma$-Lactams

Daiki Ishii, Shin-ichi Hirashima*, Kosuke Nakashima, Hiroshi Akutsu, Takaaki Sakai, Yasuyuki Matsushima, Masahiro Kawada, Tsuyoshi Miura* Tokyo University of Pharmacy and Life Sciences, 1432-1 Horinouchi, Hachioji, Tokyo 192-0392, Japan

E-mail: hirashim@toyaku.ac.jp,tmiura@toyaku.ac.jp

\title{
Contents
}

1. General Information 2

2. Preparation of Organocatalysts 2

3. Preparation of Benzoyl Acrylonitriles 2

4. Preparation of $\beta, \gamma$-Unsaturated Butenolides 2

5. General Procedure of Asymmetric Direct Vinylogous Michael Addition 2

6. Compound Characterization Date of Asymmetric Direct Vinylogpus Conjugate Addition Product (1a-r) 3

7. Synthetic Transformation to Bicyclic $\boldsymbol{\gamma}$-Lactam Derivative 5

8. X-ray Crystallographic Date 13

9. Procedure for Glam Scale Experiment 15

10. References 15

11. NMR Spectra 16

12. HPLC Chromatograms 


\section{General Information}

${ }^{1} \mathrm{H}$ NMR and ${ }^{13} \mathrm{C}$ NMR spectra were recorded on a Bruker Avance III Nanobay 400 $\mathrm{MHz}$ Spectrometer (400 MHz for ${ }^{1} \mathrm{H}$ NMR and $100 \mathrm{MHz}$ for ${ }^{13} \mathrm{C} \mathrm{NMR}$ ). The chemical shifts are expressed in ppm downfield from tetramethylsilane $(\delta=0.00)$ as an internal standard and $\mathrm{CDCl}_{3}(\delta=77.16)$. Mass Spectra were recorded by an electrospray ionization-time of flight (ESI-TOF) mass spectrometer (Micromass LCT). Specific rotations were measured on a Jasco P-2200. Melting points were obtained with Yanaco MP-J3. For thin layer chromatographic (TLC) analyses, Merck precoated TLC plates (silica gel 60 F254) were used. Flash column chromatography was performed on neutral silica gel (Kanto Silica gel 60N, 40-50 $\mu \mathrm{m}$ ).

\section{Preparation of Organocatalysts}

Organocatalysts B-G were prepared according to the literature procedure. ${ }^{1-5}$

\section{Preparation of Benzoyl Acrylonitriles}

Benzoyl acrylonitriles 1a-1r were prepared according to the literature procedure. ${ }^{6-7}$

(2E,5E)-4-Oxo-6-phenylhexa-2,5-dienenitrile (1m):<smiles>N#C/C=C/C(=O)/C=C/c1ccccc1</smiles>

Purified by silica gel chromatography with hexane and EtOAc (gradually 20:1-10:1), yellow crystal; 36\% yield (658.7 mg); m.p. 101.0-103.0 ${ }^{\circ} \mathrm{C} ;{ }^{1} \mathrm{H} \mathrm{NMR}\left(\mathrm{CDCl}_{3}, 400 \mathrm{MHz}\right): \delta 7.74(\mathrm{~d}$, $1 \mathrm{H}, J=16.0 \mathrm{~Hz}), 7.60-7.62(\mathrm{~m}, 2 \mathrm{H}), 7.42-7.47(\mathrm{~m}, 3 \mathrm{H}), 7.38$ $(\mathrm{d}, 1 \mathrm{H}, J=16.0 \mathrm{~Hz}), 6.91(\mathrm{~d}, 1 \mathrm{H}, J=16.0 \mathrm{~Hz}), 6.49(\mathrm{~d}, 1 \mathrm{H}, J=16.4 \mathrm{~Hz}) ;{ }^{13} \mathrm{C}\left\{{ }^{1} \mathrm{H}\right\} \mathrm{NMR}$ $\left(\mathrm{CDCl}_{3}, 100 \mathrm{MHz}\right): \delta 185.4,146.9,143.7,133.9,131.8,129.3,128.9,124.6,116.5,110.9$; HRMS (ESI) $m / z$ : [M+Na] $]^{+}$Calcd for $\mathrm{C}_{12} \mathrm{H}_{9} \mathrm{NONa} 206.0582$; Found 206.0574.

\section{Preparation of $\beta, \gamma$-Unsaturated Butenolides}

$\beta, \gamma$-Unsaturated butenolides $\mathbf{2 n}, \mathbf{2 0}$ and $\mathbf{2 r}$ were prepared according to the literature procedure. $^{8-9}$

\section{General Procedure of Asymmetric Direct Vinylogous Michael Addition}

$\beta, \gamma$-Butenolide 2 ( $0.24 \mathrm{mmol}, 1.2$ equiv) was added to a solution of $\beta$-cyanoenone $(0.2$ mmol, 1.0 equiv) and organocatalyst $\mathbf{G}(3.7 \mathrm{mg}, 0.006 \mathrm{mmol}, 3 \mathrm{~mol} \%)$ in dry toluene $(0.2$ $\mathrm{mL}$ ) at r.t. After stirring at r.t. for indicated time, the reaction mixture was purified by silica gel chromatography with hexane and EtOAc to afforded 3. 


\section{Compound Characterization Date of Asymmetric Direct Vinylogous Conjugate}

\section{Addition Product (1a-r)}

(R)-2-((S)-2-Methyl-5-ox0-2,5-dihydrofuran-2-yl)-4-oxo-4-phenylbutanenitrile (3a):<smiles>C[C@H](CC(=O)c1ccccc1)[C@]1(C)C=CC(=O)O1</smiles>

$3 a$

Purified by silica gel chromatography with hexane and EtOAc (gradually 10:1-3:1), white solid, 99\% yield (51.2 mg), syn/anti $>20: 1,98 \%$ ee; $[\alpha]^{28} \mathrm{D}=-131.6^{\circ}$ (c 1.00, $\left.\mathrm{CHCl}_{3}\right)$; m.p. 111.0$113.0{ }^{\circ} \mathrm{C} ;{ }^{1} \mathrm{H}$ NMR $\left(\mathrm{CDCl}_{3}, 400 \mathrm{MHz}\right): \delta 7.93(\mathrm{~d}, 2 \mathrm{H}, J=7.2$ $\mathrm{Hz}), 7.58-7.65(\mathrm{~m}, 2 \mathrm{H}), 7.48-7.52(\mathrm{~m}, 2 \mathrm{H}), 6.29(\mathrm{~d}, 1 \mathrm{H}, J=5.6$ $\mathrm{Hz}), 3.81-3.85(\mathrm{~m}, 1 \mathrm{H}), 3.30(\mathrm{~d}, 1 \mathrm{H}, J=2.4 \mathrm{~Hz}), 3.29(\mathrm{~s}, 1 \mathrm{H}), 1.70(\mathrm{~s}, 3 \mathrm{H}) ;{ }^{13} \mathrm{C}\left\{{ }^{1} \mathrm{H}\right\} \mathrm{NMR}$ $\left(\mathrm{CDCl}_{3}, 100 \mathrm{MHz}\right): \delta 194.4,170.7,156.9,135.5,134.4,129.1,128.3,123.1,118.2,86.0$, 36.1, 34.6, 23.4; Enantiomeric excess of the product was determined by chiral stationary phase HPLC analysis using a ChiralPak IC column (hexane $i$-PrOH $=75: 25$ at 1.0 $\mathrm{mL} / \mathrm{min}$ ); $\lambda=254 \mathrm{~nm} ; \mathrm{t}_{\text {major }}=39.3 \mathrm{~min}, \mathrm{t}$ minor $=44.7 \mathrm{~min}$; HRMS (ESI) $m / z:[\mathrm{M}+\mathrm{Na}]^{+}$ Calcd for $\mathrm{C}_{15} \mathrm{H}_{13} \mathrm{NO}_{3} \mathrm{Na} 278.0793$; Found 278.0786.

(R)-4-(4-Bromophenyl)-2-((S)-2-methyl-5-oxo-2,5-dihydrofuran-2-yl)-4oxobutanenitrile (3b):<smiles>C[C@]1(CC(=O)c2ccc(Br)cc2)C=CC(=O)O1</smiles>

3b

Purified by silica gel chromatography with hexane and EtOAc (gradually 10:1-3:1), white solid, 95\% yield (66.3 mg), syn/anti $>20: 1,96 \%$ ee; $[\alpha]^{27} \mathrm{D}=-100.3^{\circ}\left(\mathrm{c} 1.00, \mathrm{CHCl}_{3}\right)$; m.p. 132.0-134.0 ${ }^{\circ} \mathrm{C} ;{ }^{1} \mathrm{H}$ NMR $\left(\mathrm{CDCl}_{3}, 400 \mathrm{MHz}\right): \delta 7.78(\mathrm{~d}, 2 \mathrm{H}$, $J=8.4 \mathrm{~Hz}), 7.64(\mathrm{~d}, 2 \mathrm{H}, J=8.4 \mathrm{~Hz}), 7.56(\mathrm{~d}, 1 \mathrm{H}, J=5.6 \mathrm{~Hz})$, $6.29(\mathrm{~d}, 1 \mathrm{H}, J=5.6 \mathrm{~Hz}), 3.79-3.82(\mathrm{~m}, 1 \mathrm{H}), 3.26(\mathrm{~d}, 1 \mathrm{H}, J=4.4 \mathrm{~Hz}), 3.25(\mathrm{~s}, 1 \mathrm{H}), 1.70$ (s, 3H); ${ }^{13} \mathrm{C}\left\{{ }^{1} \mathrm{H}\right\} \mathrm{NMR}\left(\mathrm{CDCl}_{3}, 100 \mathrm{MHz}\right): \delta 193.5,170.6,156.9,134.1,132.4,129.7$, 123.1, 118.0, 85.9, 36.1, 34.6, 23.3; Enantiomeric excess of the product was determined by chiral stationary phase HPLC analysis using a ChiralPak IC column (hexane $/ i$-PrOH $=75: 25$ at $1.0 \mathrm{~mL} / \mathrm{min}) ; \lambda=254 \mathrm{~nm} ; \mathrm{t}_{\text {major }}=38.3 \mathrm{~min}, \mathrm{t}$ minor $=43.1 \mathrm{~min}$; HRMS (ESI) $m / z:[\mathrm{M}+\mathrm{Na}]^{+}$Calcd for $\mathrm{C}_{15} \mathrm{H}_{12} \mathrm{BrNO}_{3} \mathrm{Na}$ 355.9898; Found 355.9890.

\section{(R)-4-(4-Chlorophenyl)-2-((S)-2-methyl-5-oxo-2,5-dihydrofuran-2-yl)-4-} oxobutanenitrile (3c):<smiles>CC1(CCC(=O)c2ccc(Cl)cc2)C=CC(=O)O1</smiles>

$3 c$
Purified by silica gel chromatography with hexane and EtOAc (gradually 10:1-3:1), white solid, 95\% yield (55.1 $\mathrm{mg}$ ), syn/anti $>20: 1,96 \%$ ee; $[\alpha]^{28} \mathrm{D}=-119.6^{\circ}$ (c 1.00, $\left.\mathrm{CHCl}_{3}\right)$; m.p. 138.0-140.0 ${ }^{\circ} \mathrm{C} ;{ }^{1} \mathrm{H} \mathrm{NMR}\left(\mathrm{CDCl}_{3}, 400 \mathrm{MHz}\right)$ : 
$\delta 7.86(\mathrm{~d}, 2 \mathrm{H}, J=8.4 \mathrm{~Hz}), 7.59(\mathrm{~d}, 1 \mathrm{H}, J=5.6 \mathrm{~Hz}), 7.47(\mathrm{~d}, 2 \mathrm{H}, J=8.4 \mathrm{~Hz}), 6.29(\mathrm{~d}, 1 \mathrm{H}$, $J=5.6 \mathrm{~Hz}), 3.79-3.83(\mathrm{~m}, 1 \mathrm{H}), 3.25-3.27(\mathrm{~m}, 2 \mathrm{H}), 1.70(\mathrm{~s}, 3 \mathrm{H}) ;{ }^{13} \mathrm{C}\left\{{ }^{1} \mathrm{H}\right\} \mathrm{NMR}\left(\mathrm{CDCl}_{3}\right.$, $100 \mathrm{MHz}): \delta 193.3,170.6,156.9,140.9,133.7,129.6,129.4,123.1,118.0,85.9,36.1$, 34.6, 23.3; Enantiomeric excess of the product was determined by chiral stationary phase HPLC analysis using a ChiralPak IC column (hexane $/ i-\mathrm{PrOH}=75: 25$ at $1.0 \mathrm{~mL} / \mathrm{min}$ ); $\lambda$ $=254 \mathrm{~nm} ; \mathrm{t}_{\text {major }}=36.4 \mathrm{~min}, \mathrm{t}$ minor $=40.8 \mathrm{~min}$; HRMS (ESI) $\mathrm{m} / \mathrm{z}:[\mathrm{M}+\mathrm{Na}]^{+}$Calcd for $\mathrm{C}_{15} \mathrm{H}_{12} \mathrm{ClNO}_{3} \mathrm{Na} 312.0403$; Found 312.0395.

\section{(R)-2-((S)-2-Methyl-5-oxo-2,5-dihydrofuran-2-yl)-4-0xo-4-(4-}

\section{(trifluoromethyl)phenyl)butanenitrile (3d):}

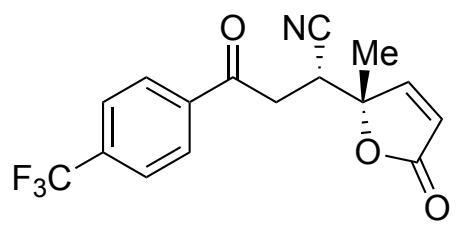

3d

Purified by silica gel chromatography with hexane and EtOAc (gradually 10:1-3:1), white solid, 94\% yield (60.7 $\mathrm{mg}$ ), syn/anti $>20: 1,93 \%$ ee; $[\alpha]^{29} \mathrm{D}=-123.9^{\circ}$ (c 1.00 , $\left.\mathrm{CHCl}_{3}\right)$; m.p. 98.0-100.0 ${ }^{\circ} \mathrm{C} ;{ }^{1} \mathrm{H} \mathrm{NMR}\left(\mathrm{CDCl}_{3}, 400 \mathrm{MHz}\right)$ : $\delta 8.03(\mathrm{~d}, 2 \mathrm{H}, J=8.2 \mathrm{~Hz}), 7.77(\mathrm{~d}, 2 \mathrm{H}, J=8.2 \mathrm{~Hz}), 7.60(\mathrm{~d}$, $1 \mathrm{H}, J=5.6 \mathrm{~Hz}), 6.30(\mathrm{~d}, 1 \mathrm{H}, J=5.6 \mathrm{~Hz}), 3.81-3.84(\mathrm{~m}, 1 \mathrm{H}), 3.31(\mathrm{~d}, 1 \mathrm{H}, J=5.2 \mathrm{~Hz})$, $3.29(\mathrm{~d}, 1 \mathrm{H}, J=2.4 \mathrm{~Hz}), 1.72(\mathrm{~s}, 3 \mathrm{H}) ;{ }^{13} \mathrm{C}\left\{{ }^{1} \mathrm{H}\right\} \mathrm{NMR}\left(\mathrm{CDCl}_{3}, 100 \mathrm{MHz}\right): \delta 193.8,170.6$, $156.9,138.0,135.4\left(\mathrm{q},{ }^{2} J_{\mathrm{C}-\mathrm{F}}=32.5 \mathrm{~Hz}\right), 128.6,126.1\left(\mathrm{q},{ }^{3} J_{\mathrm{C}-\mathrm{F}}=3.7 \mathrm{~Hz}\right), 123.4\left(\mathrm{q},{ }^{1} J_{\mathrm{C}-\mathrm{F}}=\right.$ $271.2 \mathrm{~Hz}), 123.1,118.0,85.9,36.5,34.6,23.2$; Enantiomeric excess of the product was determined by chiral stationary phase HPLC analysis using a ChiralPak AD-H column (hexane $/ i-\mathrm{PrOH}=80: 20$ at $1.0 \mathrm{~mL} / \mathrm{min}$ ) $; \lambda=254 \mathrm{~nm} ; \mathrm{t}_{\text {minor }}=13.6 \mathrm{~min}, \mathrm{t}_{\text {major }}=58.2 \mathrm{~min}$; HRMS (ESI) $m / z$ : [M+Na] $]^{+}$Calcd for $\mathrm{C}_{16} \mathrm{H}_{12} \mathrm{~F}_{3} \mathrm{NO}_{3} \mathrm{Na} 346.0667$; Found 346.0660.

\section{4-((R)-3-Cyano-3-((S)-2-methyl-5-oxo-2,5-dihydrofuran-2-} yl)propanoyl)benzonitrile (3e):<smiles>CC1(CC(=O)c2ccc(C#N)cc2)C=CC(=O)O1</smiles>

$3 e$

Purified by silica gel chromatography with hexane and EtOAc (gradually 10:1-3:1), pale yellow solid, 94\% yield (52.6 mg), syn/anti $>20: 1,91 \%$ ee; $[\alpha]^{29} \mathrm{D}=-122.3^{\circ}(\mathrm{c} 1.00$, $\left.\mathrm{CHCl}_{3}\right)$; m.p. 172.0-174.0 ${ }^{\circ} \mathrm{C} ;{ }^{1} \mathrm{H} \mathrm{NMR}\left(\mathrm{CDCl}_{3}, 400 \mathrm{MHz}\right)$ : $\delta 8.02(\mathrm{~d}, 2 \mathrm{H}, J=8.4 \mathrm{~Hz}), 7.81(\mathrm{~d}, 2 \mathrm{H}, J=8.4 \mathrm{~Hz}), 7.61(\mathrm{~d}$, $1 \mathrm{H}, J=5.6 \mathrm{~Hz}), 6.30$ (d, 1H, $J=5.6 \mathrm{~Hz}), 3.79-3.83(\mathrm{~m}, 1 \mathrm{H}), 3.28-3.31(\mathrm{~m}, 2 \mathrm{H}), 1.72(\mathrm{~s}$, $3 \mathrm{H}) ;{ }^{13} \mathrm{C}\left\{{ }^{1} \mathrm{H}\right\} \mathrm{NMR}\left(\mathrm{CDCl}_{3}, 100 \mathrm{MHz}\right): \delta 193.4,170.5,156.8,138.2,132.9,128.7,123.2$, $117.8,117.7,117.5,85.8,36.4,34.6,23.3$; Enantiomeric excess of the product was determined by chiral stationary phase HPLC analysis using a ChiralPak AS-H column (hexane $/ i-\mathrm{PrOH}=60: 40$ at $1.2 \mathrm{~mL} / \mathrm{min}$ ) $; \lambda=254 \mathrm{~nm} ; \mathrm{t}_{\text {minor }}=51.8 \mathrm{~min}, \mathrm{t}_{\text {major }}=82.0 \mathrm{~min}$; HRMS (ESI) $m / z$ : $[\mathrm{M}+\mathrm{Na}]^{+}$Calcd for $\mathrm{C}_{16} \mathrm{H}_{12} \mathrm{~N}_{2} \mathrm{O}_{3} \mathrm{Na}$ 303.0746; Found 303.0739. 
(R)-2-((S)-2-Methyl-5-oxo-2,5-dihydrofuran-2-yl)-4-oxo-4-(p-tolyl)butanenitrile (3f):<smiles>Cc1ccc(C(=O)CC(C#N)C2(C)C=CC(=O)O2)cc1</smiles>

$3 \mathbf{f}$

Purified by silica gel chromatography with hexane and EtOAc (gradually 10:1-3:1), white solid, 97\% yield (52.5 $\mathrm{mg}$ ), syn/anti $>20: 1,98 \%$ ee; $[\alpha]^{29} \mathrm{D}=-125.4^{\circ}$ (c 1.00, $\left.\mathrm{CHCl}_{3}\right)$; m.p. 128.0-130.0 ${ }^{\circ} \mathrm{C}$; ${ }^{1} \mathrm{H} \mathrm{NMR}\left(\mathrm{CDCl}_{3}, 400 \mathrm{MHz}\right)$ : $\delta 7.82(\mathrm{~d}, 2 \mathrm{H}, J=8.0 \mathrm{~Hz}), 7.59(\mathrm{~d}, 1 \mathrm{H}, J=5.6 \mathrm{~Hz}), 7.29(\mathrm{~d}$, $2 \mathrm{H}, J=8.0 \mathrm{~Hz}), 6.28(\mathrm{~d}, 1 \mathrm{H}, J=5.6 \mathrm{~Hz}), 3.82(\mathrm{t}, 1 \mathrm{H}, J=6.6 \mathrm{~Hz}), 3.27(\mathrm{~d}, 2 \mathrm{H}, J=6.8$ $\mathrm{Hz}), 2.43$ (s, 3H), 1.69 (s, 3H); ${ }^{13} \mathrm{C}\left\{{ }^{1} \mathrm{H}\right\} \mathrm{NMR}\left(\mathrm{CDCl}_{3}, 100 \mathrm{MHz}\right): \delta 194.0,170.7,157.1$, 145.3, 133.0, 129.6, 128.3, 122.9, 118.2, 86.0, 36.0, 34.6, 23.1, 21.8; Enantiomeric excess of the product was determined by chiral stationary phase HPLC analysis using a ChiralPak AD-H column (hexane $/ i-\mathrm{PrOH}=90: 10$ at $1.0 \mathrm{~mL} / \mathrm{min}$ ); $\lambda=254 \mathrm{~nm} ; \mathrm{t}$ minor $=$ $34.3 \mathrm{~min}, \mathrm{t}$ major $=68.5 \mathrm{~min}$; HRMS (ESI) $\mathrm{m} / \mathrm{z}:[\mathrm{M}+\mathrm{Na}]^{+}$Calcd for $\mathrm{C}_{16} \mathrm{H}_{15} \mathrm{NO}_{3} \mathrm{Na}$ 292.0950; Found 292.0942.

\section{(R)-2-((S)-2-Methyl-5-oxo-2,5-dihydrofuran-2-yl)-4-0xo-4-(m-tolyl)butanenitrile}

(3g):<smiles>Cc1cccc(C(=O)CCC(C)(C)C2(C)C=CC(=O)O2)c1</smiles>

$3 g$

Purified by silica gel chromatography with hexane and EtOAc (gradually 10:1-3:1), white solid, 97\% yield (52.2 $\mathrm{mg}$ ), syn/anti $>20: 1,99 \%$ ee; $[\alpha]^{25} \mathrm{D}=-121.7^{\circ}$ (c 1.00, $\left.\mathrm{CHCl}_{3}\right)$; m.p. 138.0-140.0 ${ }^{\circ} \mathrm{C} ;{ }^{1} \mathrm{H} \mathrm{NMR}\left(\mathrm{CDCl}_{3}, 400 \mathrm{MHz}\right)$ : $\delta 7.71(\mathrm{~d}, 2 \mathrm{H}, J=10.8 \mathrm{~Hz}), 7.59(\mathrm{~d}, 1 \mathrm{H}, J=5.6 \mathrm{~Hz}), 7.44(\mathrm{~d}$, $1 \mathrm{H}, J=7.6 \mathrm{~Hz}), 7.38(\mathrm{t}, 1 \mathrm{H}, J=7.6 \mathrm{~Hz}), 6.29(\mathrm{~d}, 1 \mathrm{H}, J=5.6 \mathrm{~Hz}), 3.83(\mathrm{t}, 1 \mathrm{H}, J=6.4$ $\mathrm{Hz}), 3.29(\mathrm{~d}, 1 \mathrm{H}, J=2.4 \mathrm{~Hz}), 3.28(\mathrm{~s}, 1 \mathrm{H}), 2.43(\mathrm{~s}, 3 \mathrm{H}) 1.70(\mathrm{~s}, 3 \mathrm{H}) ;{ }^{13} \mathrm{C}\left\{{ }^{1} \mathrm{H}\right\} \mathrm{NMR}$ $\left(\mathrm{CDCl}_{3}, 100 \mathrm{MHz}\right): \delta$ 194.6, 170.7, 157.1, 138.9, 135.5, 135.1, 128.9, 128.7, 125.4, 123.0, $118.2,86.0,36.2,34.6,23.2,21.4$; Enantiomeric excess of the product was determined by chiral stationary phase HPLC analysis using a ChiralCel OJ-H column (hexane $/ i$-PrOH $=75: 25$ at $1.5 \mathrm{~mL} / \mathrm{min}) ; \lambda=254 \mathrm{~nm} ; \mathrm{t}_{\text {major }}=39.5 \mathrm{~min}, \mathrm{t}$ minor $=63.5 \mathrm{~min}$; HRMS (ESI) $m / z:[\mathrm{M}+\mathrm{Na}]^{+}$Calcd for $\mathrm{C}_{16} \mathrm{H}_{15} \mathrm{NO}_{3} \mathrm{Na} 292.0950$; Found 292.0943. 


\section{(R)-2-((S)-2-Methyl-5-oxo-2,5-dihydrofuran-2-yl)-4-oxo-4-(o-tolyl)butanenitrile}

(3h):<smiles>Cc1ccccc1C(=O)C[C@@H](C#N)[C@]1(C)C=CC(=O)O1</smiles>

3h

Purified by silica gel chromatography with hexane and EtOAc (gradually 10:1-3:1), white solid, 90\% yield (49.0 mg), syn/anti $>20: 1,89 \%$ ee; $[\alpha]^{29}{ }_{\mathrm{D}}=-113.5^{\circ}$ (c 1.00, $\left.\mathrm{CHCl}_{3}\right)$; m.p. 103.0$105.0{ }^{\circ} \mathrm{C} ;{ }^{1} \mathrm{H} \mathrm{NMR}\left(\mathrm{CDCl}_{3}, 400 \mathrm{MHz}\right): \delta 7.57-7.61(\mathrm{~m}, 2 \mathrm{H})$, 7.42-7.46 (m, 1H), 7.29 (t, 2H, $J=6.4 \mathrm{~Hz}), 6.27$ (d, 1H, $J=6.0$

$\mathrm{Hz}), 3.81(\mathrm{dd}, 1 \mathrm{H}, J=5.6,7.6 \mathrm{~Hz}), 3.21(\mathrm{~d}, 1 \mathrm{H}, J=3.2 \mathrm{~Hz}), 3.19(\mathrm{~s}, 1 \mathrm{H}), 2.54(\mathrm{~s}, 3 \mathrm{H})$ $1.71(\mathrm{~s}, 3 \mathrm{H}) ;{ }^{13} \mathrm{C}\left\{{ }^{1} \mathrm{H}\right\}$ NMR $\left(\mathrm{CDCl}_{3}, 100 \mathrm{MHz}\right): \delta 197.4,170.7,156.9,139.4,135.6$, $132.6,132.5,128.8,126.1,123.0,118.3,86.0,38.4,34.8,23.3,21.7$; Enantiomeric excess of the product was determined by chiral stationary phase HPLC analysis using a ChiralPak AD-H column (hexane $/ i-\mathrm{PrOH}=80: 20$ at $1.0 \mathrm{~mL} / \mathrm{min}$ ); $\lambda=254 \mathrm{~nm} ; \mathrm{t}$ minor $=$ 9.8 min, $\mathrm{t}_{\text {major }}=18.2 \mathrm{~min}$; HRMS (ESI) $\mathrm{m} / \mathrm{z}$ : [M-H] $]^{-}$Calcd for $\mathrm{C}_{16} \mathrm{H}_{14} \mathrm{NO}_{3} 268.0974$; Found 268.0974.

(R)-4-(3,4-Dimethylphenyl)-2-((S)-2-methyl-5-oxo-2,5-dihydrofuran-2-yl)-4oxobutanenitrile (3i):<smiles>Cc1ccc(C(=O)C[C@@H](C)[C@]2(C)C=CC(=O)O2)cc1C</smiles>

$3 \mathbf{i}$

Purified by silica gel chromatography with hexane and EtOAc (gradually 10:1-3:1), white solid, 96\% yield (54.0 $\mathrm{mg})$, syn/anti $>20: 1,99 \%$ ee; $[\alpha]^{29} \mathrm{D}=-116.3^{\circ}$ (c 1.00, $\left.\mathrm{CHCl}_{3}\right)$; m.p. $110.0-112.0{ }^{\circ} \mathrm{C} ;{ }^{1} \mathrm{H} \mathrm{NMR}\left(\mathrm{CDCl}_{3}, 400 \mathrm{MHz}\right)$ : $\delta 7.69(\mathrm{~s}, 1 \mathrm{H}), 7.64(\mathrm{~d}, 1 \mathrm{H}, J=7.6 \mathrm{~Hz}), 7.58(\mathrm{~d}, 1 \mathrm{H}, J=5.6$ $\mathrm{Hz}), 7.23(\mathrm{~d}, 1 \mathrm{H}, J=8.0 \mathrm{~Hz}), 6.28(\mathrm{~d}, 1 \mathrm{H}, J=5.6 \mathrm{~Hz}), 3.83(\mathrm{t}, 1 \mathrm{H}, J=6.6 \mathrm{~Hz}), 3.28(\mathrm{~d}$, $1 \mathrm{H}, J=1.6 \mathrm{~Hz}), 3.26(\mathrm{~s}, 1 \mathrm{H}), 2.33(\mathrm{~s}, 3 \mathrm{H}), 2.32(\mathrm{~s}, 3 \mathrm{H}), 1.68(\mathrm{~s}, 3 \mathrm{H}) ;{ }^{13} \mathrm{C}\left\{{ }^{1} \mathrm{H}\right\} \mathrm{NMR}$ $\left(\mathrm{CDCl}_{3}, 100 \mathrm{MHz}\right): \delta 194.1,170.8,157.1,144.1,137.5,133.4,130.2,129.3,126.0,123.0$, $118.3,86.1,36.0,34.6,23.3,20.3,19.9$; Enantiomeric excess of the product was determined by chiral stationary phase HPLC analysis using a ChiralPak AD-H column (hexane $/ i-\mathrm{PrOH}=90: 10$ at $1.0 \mathrm{~mL} / \mathrm{min}$ ); $\lambda=254 \mathrm{~nm} ; \mathrm{t}_{\text {minor }}=24.9 \mathrm{~min}, \mathrm{t}$ major $=35.9 \mathrm{~min}$; HRMS (ESI) $m / z$ : [M+Na $]^{+}$Calcd for $\mathrm{C}_{17} \mathrm{H}_{17} \mathrm{NO}_{3} \mathrm{Na} 306.1106$; Found 306.1100.

\section{(R)-4-(4-Methoxyphenyl)-2-((S)-2-methyl-5-oxo-2,5-dihydrofuran-2-yl)-4-} oxobutanenitrile (3j):<smiles>COc1ccc(C(=O)C[C@@H](C)[C@]2(C)C=CC(=O)O2)cc1</smiles>

3j
Purified by silica gel chromatography with hexane and EtOAc (gradually 10:1-3:1), white solid, 97\% yield (55.2 $\mathrm{mg})$, syn/anti $>20: 1,98 \%$ ee; $[\alpha]^{29} \mathrm{D}=-139.1^{\circ}$ (c 1.00, $\left.\mathrm{CHCl}_{3}\right)$; m.p. 102.0-104.0 ${ }^{\circ} \mathrm{C} ;{ }^{1} \mathrm{H} \mathrm{NMR}\left(\mathrm{CDCl}_{3}, 400 \mathrm{MHz}\right)$ : 
$\delta 7.90(\mathrm{~d}, 2 \mathrm{H}, J=8.8 \mathrm{~Hz}), 7.58(\mathrm{~d}, 1 \mathrm{H}, J=5.6 \mathrm{~Hz}), 6.95(\mathrm{~d}, 2 \mathrm{H}, J=8.8 \mathrm{~Hz}), 6.28(\mathrm{~d}, 1 \mathrm{H}$, $J=5.6 \mathrm{~Hz}), 3.89$ (s, 3H), $3.83(\mathrm{dd}, 1 \mathrm{H}, J=6.0,7.2 \mathrm{~Hz}), 3.25(\mathrm{~d}, 1 \mathrm{H}, J=1.2 \mathrm{~Hz}), 3.24$ (s, $1 \mathrm{H}), 1.69(\mathrm{~s}, 3 \mathrm{H}) ;{ }^{13} \mathrm{C}\left\{{ }^{1} \mathrm{H}\right\} \mathrm{NMR}\left(\mathrm{CDCl}_{3}, 100 \mathrm{MHz}\right): \delta 192.7,170.8,164.4,157.1,130.6$, $128.5,122.9,118.3,114.2,86.1,55.7,35.7,34.7,23.1$; Enantiomeric excess of the product was determined by chiral stationary phase HPLC analysis using a ChiralPak AD$\mathrm{H}$ column (hexane $/ i-\mathrm{PrOH}=80: 20$ at $1.0 \mathrm{~mL} / \mathrm{min}$ ); $\lambda=254 \mathrm{~nm}$; $\mathrm{t}_{\text {minor }}=23.6 \mathrm{~min}, \mathrm{t}$ major $=41.4 \mathrm{~min}$; HRMS (ESI) $m / z:[\mathrm{M}+\mathrm{Na}]^{+}$Calcd for $\mathrm{C}_{16} \mathrm{H}_{15} \mathrm{NO}_{4} \mathrm{Na}$ 308.0899; Found 308.0892 .

\section{(R)-4-(Furan-2-yl)-2-((S)-2-methyl-5-oxo-2,5-dihydrofuran-2-yl)-4-} oxobutanenitrile (3k):

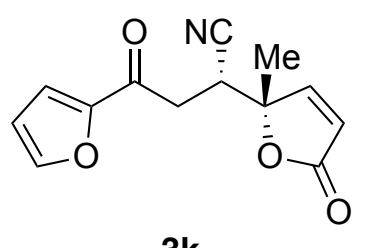

Purified by silica gel chromatography with hexane and EtOAc (gradually 10:1-3:1), pale yellow solid, 96\% yield $(50.1 \mathrm{mg}$ ), syn/anti $>20: 1,93 \%$ ee; $[\alpha]^{25} \mathrm{D}=-88.0^{\circ}$ (c 0.50, $\left.\mathrm{CHCl}_{3}\right) ;$ m.p. 158.0-160.0 ${ }^{\circ} \mathrm{C} ;{ }^{1} \mathrm{H} \mathrm{NMR}\left(\mathrm{CDCl}_{3}, 400 \mathrm{MHz}\right): \delta 7.62(\mathrm{dd}, 1 \mathrm{H}, J=$ $0.8,2.0 \mathrm{~Hz}), 7.57(\mathrm{~d}, 1 \mathrm{H}, J=5.6 \mathrm{~Hz}), 7.29(\mathrm{dd}, 1 \mathrm{H}, J=0.8,3.6$ $\mathrm{Hz}), 6.59(\mathrm{dd}, 1 \mathrm{H}, J=1.6,3.6 \mathrm{~Hz}), 6.28(\mathrm{~d}, 1 \mathrm{H}, J=5.6 \mathrm{~Hz}), 3.79(\mathrm{dd}, 1 \mathrm{H}, J=5.4,8.2$ $\mathrm{Hz}), 3.14-3.17(\mathrm{~m}, 2 \mathrm{H}) 1.70(\mathrm{~s}, 3 \mathrm{H}) ;{ }^{13} \mathrm{C}\left\{{ }^{1} \mathrm{H}\right\} \mathrm{NMR}\left(\mathrm{CDCl}_{3}, 100 \mathrm{MHz}\right): \delta 183.4,170.7$, 156.8, 151.6, 147.4, 123.2, 118.5, 117.9, 113.0, 86.0, 35.7, 34.2, 23.2; Enantiomeric excess of the product was determined by chiral stationary phase HPLC analysis using a ChiralPak AD-H column (hexane $/ i-\mathrm{PrOH}=90: 10$ at $1.0 \mathrm{~mL} / \mathrm{min}$ ); $\lambda=254 \mathrm{~nm} ; \mathrm{t}$ minor $=$ 38.9 min, $\mathrm{t}$ major $=101.6 \mathrm{~min}$; HRMS (ESI) $\mathrm{m} / \mathrm{z}:[\mathrm{M}+\mathrm{Na}]^{+}$Calcd for $\mathrm{C}_{13} \mathrm{H}_{11} \mathrm{NO}_{4} \mathrm{Na}$ 268.0586; Found 268.0579.

\section{(R)-2-((S)-2-Methyl-5-oxo-2,5-dihydrofuran-2-yl)-4-oxo-4-(thiophen-2-}

\section{yl)butanenitrile (3l):}

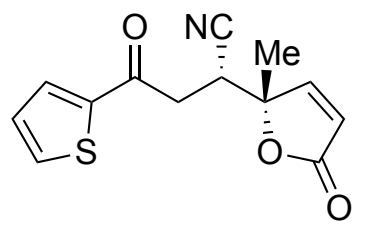

Purified by silica gel chromatography with hexane and EtOAc (gradually 10:1-3:1), pale yellow solid, 91\% yield (47.4 mg), syn/anti $>20: 1,97 \%$ ee; $[\alpha]^{25} \mathrm{D}=-204.6^{\circ}\left(\mathrm{c} 0.50, \mathrm{CHCl}_{3}\right)$; m.p.

O $172.0-174.0{ }^{\circ} \mathrm{C} ;{ }^{1} \mathrm{H}$ NMR $\left(\mathrm{CDCl}_{3}, 400 \mathrm{MHz}\right): \delta 7.73(\mathrm{~d}, 2 \mathrm{H}, J=$ 3I $3.6 \mathrm{~Hz}), 7.59$ (d, $1 \mathrm{H}, J=5.6 \mathrm{~Hz}), 7.16-7.18(\mathrm{~m}, 1 \mathrm{H}), 6.28(\mathrm{~d}, 1 \mathrm{H}$, $J=5.6 \mathrm{~Hz}), 3.80-3.83(\mathrm{~m}, 1 \mathrm{H}), 3.27(\mathrm{~d}, 1 \mathrm{H}, J=3.2 \mathrm{~Hz}), 3.26(\mathrm{~s}, 1 \mathrm{H}), 1.68(\mathrm{~s}, 3 \mathrm{H})$; ${ }^{13} \mathrm{C}\left\{{ }^{1} \mathrm{H}\right\} \mathrm{NMR}\left(\mathrm{CDCl}_{3}, 100 \mathrm{MHz}\right): \delta 187.1,170.7,157.0,142.3,135.3,133.0,128.6$, 123.0, 117.9, 86.0, 36.5, 34.5, 23.1; Enantiomeric excess of the product was determined by chiral stationary phase HPLC analysis using a ChiralPak AD-H column (hexane $/ i$ $\mathrm{PrOH}=80: 20$ at $1.0 \mathrm{~mL} / \mathrm{min}) ; \lambda=254 \mathrm{~nm}$; $\mathrm{t}_{\text {minor }}=16.0 \mathrm{~min}, \mathrm{t}_{\text {major }}=37.4 \mathrm{~min}$; HRMS 
(ESI) $m / z$ : $[\mathrm{M}+\mathrm{Na}]^{+}$Calcd for $\mathrm{C}_{13} \mathrm{H}_{11} \mathrm{NO}_{3} \mathrm{SNa} 284.0357$; Found 284.0349.

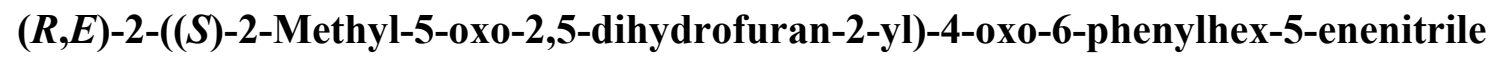
(3m):<smiles>CC(CC(=O)/C=C/c1ccccc1)C1(C)C=CC(=O)O1</smiles>

$3 m$

Purified by silica gel chromatography with hexane and EtOAc (gradually 10:1-3:1), white solid, 90\% yield (50.5 $\mathrm{mg}$ ), syn/anti $=16: 1,94 \%$ ee; $[\alpha]^{29} \mathrm{D}=-87.7^{\circ}$ (c 1.00, $\left.\mathrm{CHCl}_{3}\right)$; m.p. 129.0-131.0 ${ }^{\circ} \mathrm{C} ;{ }^{1} \mathrm{H} \mathrm{NMR}\left(\mathrm{CDCl}_{3}, 400 \mathrm{MHz}\right)$ : $\delta 7.61(\mathrm{~d}, 1 \mathrm{H}, J=16.2 \mathrm{~Hz}), 7.55-7.57(\mathrm{~m}, 3 \mathrm{H}), 7.41-7.44(\mathrm{~m}$, $3 \mathrm{H}), 6.74(\mathrm{~d}, 1 \mathrm{H}, J=16.2 \mathrm{~Hz}), 6.28(\mathrm{~d}, 1 \mathrm{H}, J=5.6 \mathrm{~Hz}), 3.74(\mathrm{t}, 1 \mathrm{H}, J=6.6 \mathrm{~Hz}), 2.99(\mathrm{~d}$, $2 \mathrm{H}, J=6.8 \mathrm{~Hz}), 1.68(\mathrm{~s}, 3 \mathrm{H}) ;{ }^{13} \mathrm{C}\left\{{ }^{1} \mathrm{H}\right\} \mathrm{NMR}\left(\mathrm{CDCl}_{3}, 100 \mathrm{MHz}\right): \delta$ 194.0, 170.7, 157.0, $144.8,133.8,131.3,129.2,128.7,124.6,122.9,118.1,86.0,37.9,34.4,23.1$; Enantiomeric excess of the product was determined by chiral stationary phase HPLC analysis using a ChiralPak AD-H column (hexane $/ i-\mathrm{PrOH}=80: 20$ at $1.0 \mathrm{~mL} / \mathrm{min}$ ) $; \lambda=$ $254 \mathrm{~nm} ; \mathrm{t}$ minor $=25.3 \mathrm{~min}, \mathrm{t}$ major $=33.8 \mathrm{~min}$; HRMS (ESI) $\mathrm{m} / z:[\mathrm{M}-\mathrm{H}]^{-}$Calcd for $\mathrm{C}_{17} \mathrm{H}_{14} \mathrm{NO}_{3} 280.0974$; Found 280.0971.

(R)-2-((S)-2-Ethyl-5-oxo-2,5-dihydrofuran-2-yl)-4-0xo-4-phenylbutanenitrile (3n):<smiles>CCC1(CCC(=O)c2ccccc2)C=CC(=O)O1</smiles>

$3 n$ Purified by silica gel chromatography with hexane and EtOAc (gradually 10:1-3:1), white solid, 94\% yield (52.9 mg), syn/anti $>20: 1,97 \%$ ee; $[\alpha]^{29} \mathrm{D}=-135.4^{\circ}$ (c 1.00, $\left.\mathrm{CHCl}_{3}\right)$; m.p. 108.0$110.0{ }^{\circ} \mathrm{C} ;{ }^{1} \mathrm{H} \mathrm{NMR}\left(\mathrm{CDCl}_{3}, 400 \mathrm{MHz}\right): \delta 7.90-7.92(\mathrm{~m}, 2 \mathrm{H}), 7.60-$ $7.64(\mathrm{~m}, 1 \mathrm{H}), 7.47-7.52(\mathrm{~m}, 3 \mathrm{H}), 6.33(\mathrm{~d}, 1 \mathrm{H}, J=6.0 \mathrm{~Hz}), 3.88$ (dd, $1 \mathrm{H}, J=4.8,8.4 \mathrm{~Hz}), 3.24-3.27(\mathrm{~m}, 2 \mathrm{H}), 2.01-2.18(\mathrm{~m}, 2 \mathrm{H}), 0.94$ (t, 3H, $J=7.6 \mathrm{~Hz}$ ); ${ }^{13} \mathrm{C}\left\{{ }^{1} \mathrm{H}\right\} \mathrm{NMR}\left(\mathrm{CDCl}_{3}, 100 \mathrm{MHz}\right): \delta 194.4,171.0,155.9,135.5,134.2,129.0,128.2$, 124.0, 118.3, 88.7, 35.9, 33.3, 28.8, 7.5; Enantiomeric excess of the product was determined by chiral stationary phase HPLC analysis using a ChiralPak AD-H column (hexane $/ i-\mathrm{PrOH}=80: 20$ at $1.0 \mathrm{~mL} / \mathrm{min}$ ) $; \lambda=254 \mathrm{~nm} ; \mathrm{t}_{\text {minor }}=11.1 \mathrm{~min}, \mathrm{t}_{\text {major }}=19.8 \mathrm{~min}$; HRMS (ESI) $m / z$ : [M+Na $]^{+}$Calcd for $\mathrm{C}_{16} \mathrm{H}_{15} \mathrm{NO}_{3} \mathrm{Na} 292.0950$; Found 292.0942.

\section{(R)-2-((S)-2-Benzyl-5-oxo-2,5-dihydrofuran-2-yl)-4-oxo-4-phenylbutanenitrile (3o):}<smiles>N#C[C@@]1(CCC(=O)c2ccccc2)C=CC(=O)O1</smiles>

30

Purified by silica gel chromatography with hexane and EtOAc (gradually 10:1-3:1), white solid, 93\% yield (61.6 mg), syn/anti > $20: 1,96 \%$ ee; $[\alpha]^{29} \mathrm{D}=-115.4^{\circ}\left(\mathrm{c} 1.00, \mathrm{CHCl}_{3}\right)$; m.p. 175.0-177.0

${ }^{\circ} \mathrm{C} ;{ }^{1} \mathrm{H}$ NMR $\left(\mathrm{CDCl}_{3}, 400 \mathrm{MHz}\right): \delta$ 7.87-7.90 (m, 2H), 7.59-7.63 $(\mathrm{m}, 1 \mathrm{H}), 7.56(\mathrm{~d}, 1 \mathrm{H}, J=5.6 \mathrm{~Hz}), 7.46-7.50(\mathrm{~m}, 2 \mathrm{H}), 7.27-7.34$ 
(m, 3H), 7.20-7.23 (m, 2H), $6.13(\mathrm{~d}, 1 \mathrm{H}, J=5.6 \mathrm{~Hz}), 3.83(\mathrm{dd}, 1 \mathrm{H}, J=5.4,8.2 \mathrm{~Hz}), 3.23-$ $3.40(\mathrm{~m}, 4 \mathrm{H}) ;{ }^{13} \mathrm{C}\left\{{ }^{1} \mathrm{H}\right\}$ NMR $\left(\mathrm{CDCl}_{3}, 100 \mathrm{MHz}\right): \delta 194.3,170.5,155.8,135.5,134.3$, $132.5, \quad 130.5, \quad 129.0, \quad 128.8, \quad 128.2, \quad 128.0, \quad 124.1, \quad 118.4, \quad 88.1, \quad 42.3, \quad 35.8$, 33.1; Enantiomeric excess of the product was determined by chiral stationary phase HPLC analysis using a ChiralPak AD-H column (hexane $/ i-\mathrm{PrOH}=85: 15$ at $1.0 \mathrm{~mL} / \mathrm{min}$ ); $\lambda=254 \mathrm{~nm} ; \mathrm{t}_{\text {minor }}=19.5 \mathrm{~min}, \mathrm{t}$ major $=31.2 \mathrm{~min}$; HRMS (ESI) $\mathrm{m} / z:[\mathrm{M}+\mathrm{Na}]^{+}$Calcd for $\mathrm{C}_{21} \mathrm{H}_{17} \mathrm{NO}_{3} \mathrm{Na} 354.1106$; Found 354.1100.

\section{(R)-2-((S)-2-Benzyl-5-oxo-2,5-dihydrofuran-2-yl)-4-(4-chlorophenyl)-4-} oxobutanenitrile (3p):<smiles>N#C[C@@H](CC(=O)c1ccc(Cl)cc1)[C@]1(Br)C=CC(=O)O1</smiles>

$3 p$

Purified by silica gel chromatography with hexane and EtOAc (gradually 10:1-3:1), white solid, 90\% yield (65.5 $\mathrm{mg})$, syn/anti $>20: 1,94 \%$ ee; $[\alpha]^{29}{ }_{\mathrm{D}}=-75.4^{\circ}\left(\mathrm{c} 1.00, \mathrm{CHCl}_{3}\right)$; m.p. 162.0-164.0 ${ }^{\circ} \mathrm{C} ;{ }^{1} \mathrm{H} \mathrm{NMR}\left(\mathrm{CDCl}_{3}, 400 \mathrm{MHz}\right): \delta 7.82(\mathrm{~d}$, $2 \mathrm{H}, J=8.4 \mathrm{~Hz}), 7.56(\mathrm{~d}, 1 \mathrm{H}, J=5.6 \mathrm{~Hz}), 7.45(\mathrm{~d}, 2 \mathrm{H}, J=8.4$ Hz), 7.29-7.32 (m, 3H), 7.20-7.22 (m, 2H), 6.14 (d, 1H, $J=5.6 \mathrm{~Hz}), 3.79-3.83(\mathrm{~m}, 1 \mathrm{H})$, 3.18-3.40 (m, 4H); ${ }^{13} \mathrm{C}\left\{{ }^{1} \mathrm{H}\right\} \mathrm{NMR}\left(\mathrm{CDCl}_{3}, 100 \mathrm{MHz}\right): \delta 193.1,170.5,155.7,140.9,133.8$, 132.4, 130.5, 129.6, 129.4, 128.9, 128.1, 124.2, 118.3, 88.1, 42.4, 35.7, 33.1; Enantiomeric excess of the product was determined by chiral stationary phase HPLC analysis using a ChiralPak AD-H column (hexane $/ i-\mathrm{PrOH}=85: 15$ at $1.0 \mathrm{~mL} / \mathrm{min}$ ); $\lambda=$ $254 \mathrm{~nm} ; \mathrm{t}_{\text {minor }}=22.6 \mathrm{~min}, \mathrm{t}$ major $=42.2 \mathrm{~min}$; HRMS (ESI) $\mathrm{m} / \mathrm{z}:[\mathrm{M}+\mathrm{Na}]^{+}$Calcd for $\mathrm{C}_{21} \mathrm{H}_{16} \mathrm{ClNO}_{3} \mathrm{Na} 388.0716$; Found 388.0710.

\section{(R)-2-((S)-2-Benzyl-5-oxo-2,5-dihydrofuran-2-yl)-4-(4-bromophenyl)-4-} oxobutanenitrile (3q):<smiles>N#C[C@@H](CC(=O)c1ccc(Br)cc1)[C@]1(Br)C=CC(=O)O1</smiles>

$3 q$

Purified by silica gel chromatography with hexane and EtOAc (gradually 10:1-3:1), white solid, 89\% yield $(72.2$ $\mathrm{mg})$, syn/anti $>20: 1,94 \%$ ee; $[\alpha]^{25}{ }_{\mathrm{D}}=-66.9^{\circ}\left(\mathrm{c} 1.00, \mathrm{CHCl}_{3}\right)$; m.p. 161.0-163.0 ${ }^{\circ} \mathrm{C} ;{ }^{1} \mathrm{H} \mathrm{NMR}\left(\mathrm{CDCl}_{3}, 400 \mathrm{MHz}\right): \delta 7.74(\mathrm{~d}$, $2 \mathrm{H}, J=8.8 \mathrm{~Hz}), 7.62(\mathrm{~d}, 2 \mathrm{H}, J=8.8 \mathrm{~Hz}), 7.56(\mathrm{~d}, 1 \mathrm{H}, J=5.6$ $\mathrm{Hz}), 7.28-7.34$ (m, 3H), 7.20-7.22 (m, 2H), 6.14 (d, $1 \mathrm{H}, J=5.6 \mathrm{~Hz}), 3.79-3.82(\mathrm{~m}, 1 \mathrm{H})$, 3.17-3.40 (m, 4H); ${ }^{13} \mathrm{C}\left\{{ }^{1} \mathrm{H}\right\} \mathrm{NMR}\left(100 \mathrm{MHz}, \mathrm{CDCl}_{3}\right): \delta 193.4,170.5,155.7,134.2,132.4$, $130.5,129.7,128.9,128.1,124.2,118.2,88.0,42.4,35.7,33.1$; Enantiomeric excess of the product was determined by chiral stationary phase HPLC analysis using a ChiralPak AD-H column (hexane $/ i-\mathrm{PrOH}=85: 15$ at $1.0 \mathrm{~mL} / \mathrm{min}$ ); $\lambda=254 \mathrm{~nm} ; \mathrm{t}$ minor $=26.1 \mathrm{~min}, \mathrm{t}$ major $=49.7 \mathrm{~min}$; HRMS (ESI) $m / z:[\mathrm{M}+\mathrm{Na}]^{+}$Calcd for $\mathrm{C}_{21} \mathrm{H}_{16} \mathrm{BrNO}_{3} \mathrm{Na} 432.0211$; Found 
(R)-2-((S)-2,4-Dimethyl-5-oxo-2,5-dihydrofuran-2-yl)-4-oxo-4-phenylbutanenitrile (3r):<smiles>CC1=C[C@](C)([C@H](C)CC(=O)c2ccccc2)OC1=O</smiles>

Purified by silica gel chromatography with hexane and EtOAc (gradually 10:1-3:1), white solid, 94\% yield $(50.5$ $\mathrm{mg})$, syn/anti $>20: 1,93 \%$ ee; $[\alpha]^{23} \mathrm{D}=-87.4^{\circ}(\mathrm{c} 1.00$, $\left.\mathrm{CHCl}_{3}\right)$; m.p. 95.0-97.0 ${ }^{\circ} \mathrm{C} ;{ }^{1} \mathrm{H} \mathrm{NMR}\left(\mathrm{CDCl}_{3}, 400 \mathrm{MHz}\right): \delta$ $7.93(\mathrm{dd}, 2 \mathrm{H}, J=1.2,8.0 \mathrm{~Hz}), 7.60-7.65(\mathrm{~m}, 1 \mathrm{H}), 7.48-7.52$ $(\mathrm{m}, 2 \mathrm{H}), 7.16(\mathrm{~d}, 1 \mathrm{H}, J=1.6 \mathrm{~Hz}), 3.76-3.79(\mathrm{~m}, 1 \mathrm{H}), 3.28-3.31(\mathrm{~m}, 2 \mathrm{H}), 2.01(\mathrm{~d}, 3 \mathrm{H}, J=$ $1.2 \mathrm{~Hz}), 1.64(\mathrm{~s}, 3 \mathrm{H}) ;{ }^{13} \mathrm{C}\left\{{ }^{1} \mathrm{H}\right\} \mathrm{NMR}\left(\mathrm{CDCl}_{3}, 100 \mathrm{MHz}\right): \delta$ 194.6, 172.1, 149.4, 135.5, $134.3,131.9,129.0,128.2,118.4,83.8,36.2,34.9,23.4,10.8$; Enantiomeric excess of the product was determined by chiral stationary phase HPLC analysis using a ChiralPak AD$\mathrm{H}$ column (hexane $/ i-\mathrm{PrOH}=90: 10$ at $1.0 \mathrm{~mL} / \mathrm{min}$ ); $\lambda=254 \mathrm{~nm}$; $\mathrm{t}_{\text {minor }}=18.7 \mathrm{~min}, \mathrm{t}$ major $=30.3$ min; HRMS (ESI) $m / z:[\mathrm{M}+\mathrm{Na}]^{+}$Calcd for $\mathrm{C}_{16} \mathrm{H}_{15} \mathrm{NO}_{3} \mathrm{Na}$ 292.0950; Found 292.0941 .

\section{Synthetic Transformation to Bicyclic $\gamma$-Lactam Derivative 5}

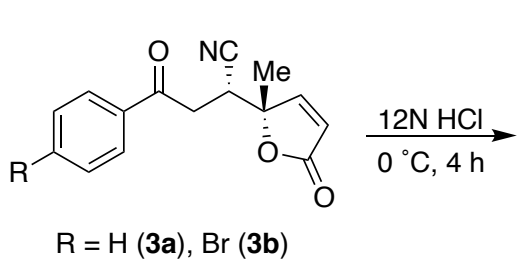

$\mathrm{R}=\mathrm{H}(\mathbf{3} \mathbf{a}), \mathrm{Br}(\mathbf{3 b})$

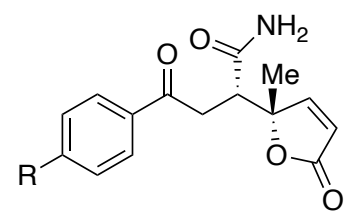

4a, $94 \%, 96 \%$ ee $(\mathrm{R}=\mathrm{H})$ 4b, $78 \%, 95 \%$ ee $(R=B r)$
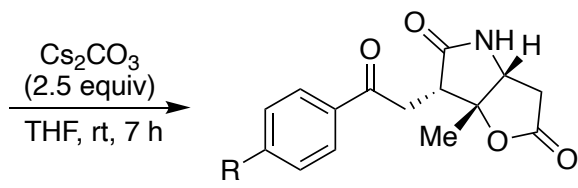

$5 a, 89 \%, 96 \%$ ee $(\mathrm{R}=\mathrm{H})$ 5b, $44 \% 95 \%$ ee $(R=B r)$

\section{Transformation of 3 into 4}

Addition product $3(1.0 \mathrm{mmol})$ was placed in a flask and cooled to $0^{\circ} \mathrm{C}$ in ice water bath. Concentrated $\mathrm{HCl}(10 \mathrm{~mL})$ was then added slowly at $0^{\circ} \mathrm{C}$ and the mixture was allowed to stir for $4 \mathrm{~h}$. The mixture was poured into ice-cold water and extracted three times with $\mathrm{CHCl}_{3}$. The organic layers were combined, washed with brine, dried over anhydrous $\mathrm{Na}_{2} \mathrm{SO}_{4}$, and concentrated in vacuo. The residue was purified by silica gel chromatography with $\mathrm{CHCl}_{3}$ and EtOAc to afforded compound 4. 
(S)-2-((S)-2-methyl-5-oxo-2,5-dihydrofuran-2-yl)-4-oxo-4-phenylbutanamide (4a):<smiles>C[C@]1(CC(=O)c2ccccc2)C=CC(=O)O1</smiles>

$4 a$

Purified by silica gel chromatography with $\mathrm{CHCl}_{3}$ and EtOAc

(gradually 8:1-1:1), yellow solid, 94\% yield (257.7 mg), syn/anti $>20: 1,96 \%$ ee; $[\alpha]^{24}{ }_{\mathrm{D}}=-142.4^{\circ}$ (c 1.00, $\left.\mathrm{CHCl}_{3}\right)$; m.p. 162.0$164.0{ }^{\circ} \mathrm{C} ;{ }^{1} \mathrm{H} \mathrm{NMR}\left(\mathrm{CDCl}_{3}, 400 \mathrm{MHz}\right): \delta 7.83-7.87(\mathrm{~m}, 3 \mathrm{H})$, 7.54-7.57 (m, 1H), 7.42 (t, 2H, $J=7.4 \mathrm{~Hz}), 6.59$ (brs, $1 \mathrm{H}), 6.16$ (d, 1H, $J=5.2 \mathrm{~Hz}), 5.83$ (brs, $1 \mathrm{H}), 3.54(\mathrm{~d}, 1 \mathrm{H}, J=10.4 \mathrm{~Hz}), 3.24-3.31(\mathrm{~m}, 1 \mathrm{H}), 2.86$ (d, $1 \mathrm{H}, J=18.0 \mathrm{~Hz}) 1.62(\mathrm{~s}, 3 \mathrm{H}) ;{ }^{13} \mathrm{C}\left\{{ }^{1} \mathrm{H}\right\} \mathrm{NMR}\left(\mathrm{CDCl}_{3}, 100 \mathrm{MHz}\right): \delta 197.8,173.8,172.0$, 159.8, 136.1, 133.9, 128.8, 128.2, 121.3, 87.8, 48.5, 36.6, 23.5; Enantiomeric excess of the product was determined by chiral stationary phase HPLC analysis using a ChiralPak AD-H column (hexane $/ i-\mathrm{PrOH}=80: 20$ at $1.0 \mathrm{~mL} / \mathrm{min}$ ); $\lambda=254 \mathrm{~nm} ; \mathrm{t}$ minor $=11.0 \mathrm{~min}, \mathrm{t}$ major $=15.8 \mathrm{~min}$; HRMS (ESI) m/z: [M-H] $]^{-}$Calcd for $\mathrm{C}_{15} \mathrm{H}_{14} \mathrm{NO}_{4}$ 272.0923; Found 272.0921 .

\section{(S)-4-(4-bromophenyl)-2-((S)-2-methyl-5-oxo-2,5-dihydrofuran-2-yl)-4-} oxobutanamide (4b):<smiles>C[C@]1(C(CC(=O)c2ccc(Br)cc2)C(N)=O)C=CC(=O)O1</smiles>

Purified by silica gel chromatography with $\mathrm{CHCl}_{3}$ and EtOAc (gradually 10:1-1:1), yellow solid, $78 \%$ yield $(284.5 \mathrm{mg}$ ), syn/anti $>20: 1,95 \%$ ee; $[\alpha]^{25} \mathrm{D}=-125.7^{\circ}\left(\mathrm{c} 0.50, \mathrm{CHCl}_{3}\right)$; m.p. 180.0-182.0 ${ }^{\circ} \mathrm{C} ;{ }^{1} \mathrm{H} \mathrm{NMR}\left(\mathrm{CDCl}_{3}, 400 \mathrm{MHz}\right): \delta 7.86(\mathrm{~d}, 1 \mathrm{H}$, $J=4.8 \mathrm{~Hz}), 7.70(\mathrm{~d}, 2 \mathrm{H}, J=8.0 \mathrm{~Hz}), 7.57(\mathrm{~d}, 2 \mathrm{H}, J=7.6 \mathrm{~Hz})$, 6.37 (brs, 1H), 6.18 (d, 1H, $J=5.6 \mathrm{~Hz}), 5.65$ (brs, 1H), 3.51 (d, 1H, $J=10.8 \mathrm{~Hz}), 3.21$ $(\mathrm{dd}, 1 \mathrm{H}, J=10.8,17.6 \mathrm{~Hz}), 2.81(\mathrm{~d}, 1 \mathrm{H}, J=18.0 \mathrm{~Hz}), 1.63(\mathrm{~s}, 3 \mathrm{H}) ;{ }^{13} \mathrm{C}\left\{{ }^{1} \mathrm{H}\right\} \mathrm{NMR}\left(\mathrm{CDCl}_{3}\right.$, $100 \mathrm{MHz}): \delta 196.7,173.5,171.9,159.7,134.8,132.2$, 129.6, 129.2, 121.4, 87.7, 48.5, $36.5,23.5$; Enantiomeric excess of the product was determined by chiral stationary phase HPLC analysis using a ChiralPak AD-H column (hexane $/ i-\mathrm{PrOH}=85: 15$ at $1.0 \mathrm{~mL} / \mathrm{min}$ ); $\lambda=254 \mathrm{~nm} ; \mathrm{t}$ minor $=22.5 \mathrm{~min}, \mathrm{t}$ major $=33.0 \mathrm{~min}$; HRMS (ESI) $\mathrm{m} / \mathrm{z}:[\mathrm{M}-\mathrm{H}]^{-}$Calcd for $\mathrm{C}_{15} \mathrm{H}_{13} \mathrm{BrNO}_{4} 350.0028$; Found 350.0022 .

\section{Transformation of 4 into 5}

Cesium carbonate (4a: $0.5 \mathrm{mmol}, \mathbf{4 b}: 1.25 \mathrm{mmol}, 2.5$ equiv) was added to a solution of 4 (4a: $0.2 \mathrm{mmol}, 4 \mathbf{b}: 0.5 \mathrm{mmol} 1.0$ equiv) in dry THF (4a: $4 \mathrm{~mL}, \mathbf{4 b}: 10 \mathrm{~mL}$ ). After stirring at r.t. for $7 \mathrm{~h}$. The mixture was added $\mathrm{NH}_{4} \mathrm{Cl}$ aq. and extracted three times with EtOAc. The organic layers were combined, washed with brine, dried over anhydrous $\mathrm{Na}_{2} \mathrm{SO}_{4}$, and concentrated in vacuo. The residue was purified by silica gel chromatography with $\mathrm{CHCl}_{3}$ and EtOAc to afforded bicyclic $\gamma$-lactam derivative $\mathbf{5 .}$ 
(3aR,6S,6aR)-6a-Methyl-6-(2-oxo-2-phenylethyl)tetrahydro-2H-furo[3,2-b]pyrrole2,5(3H)-dione (5a):

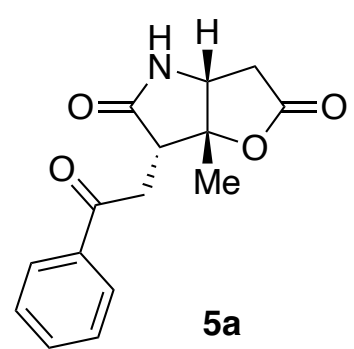

Purified by silica gel chromatography with $\mathrm{CHCl}_{3}$ and EtOAc (gradually 5:1-1:1), yellow solid, $89 \%$ yield (48.7 mg), syn/anti $>$ 20:1, 96\% ee; $[\alpha]^{25} \mathrm{D}=+98.7^{\circ}\left(\mathrm{c} 1.00, \mathrm{CHCl}_{3}\right)$; m.p. $61.0-63.0{ }^{\circ} \mathrm{C}$; ${ }^{1} \mathrm{H} \mathrm{NMR}\left(\mathrm{CDCl}_{3}, 400 \mathrm{MHz}\right): \delta 8.03(\mathrm{~d}, 2 \mathrm{H}, J=8.0 \mathrm{~Hz}), 7.60(\mathrm{t}$, $1 \mathrm{H}, J=7.4 \mathrm{~Hz}$ ), 7.47-7.51 (m, 2H), 7.31 (brs, 1H), 4.13 (d, 1H, $J$

$(\mathrm{d}, 1 \mathrm{H}, J=18.4 \mathrm{~Hz}), 1.58(\mathrm{~s}, 3 \mathrm{H}) ;{ }^{13} \mathrm{C}\left\{{ }^{1} \mathrm{H}\right\} \mathrm{NMR}\left(\mathrm{CDCl}_{3}, 100 \mathrm{MHz}\right): \delta 197.4,176.5$, 173.4, 136.4, 133.7, 128.8, 128.4, 89.3, 57.8, 45.6, 36.0, 34.3, 22.9; Enantiomeric excess of the product was determined by chiral stationary phase HPLC analysis using a ChiralPak AD-H column (hexane $/ i-\mathrm{PrOH}=80: 20$ at $1.0 \mathrm{~mL} / \mathrm{min}$ ); $\lambda=254 \mathrm{~nm} ; \mathrm{t}$ major $=$ 11.5 min, $\mathrm{t}$ minor $=18.1 \mathrm{~min}$; HRMS (ESI) $\mathrm{m} / \mathrm{z}:[\mathrm{M}+\mathrm{Na}]^{+}$Calcd for $\mathrm{C}_{15} \mathrm{H}_{15} \mathrm{NO}_{4} \mathrm{Na}$ 296.0899; Found 296. 0896.

(3aR,6S,6aR)-6-(2-(4-Bromophenyl)-2-oxoethyl)-6a-methyltetrahydro-2H-furo[3,2b]pyrrole-2,5(3H)-dione (5b):

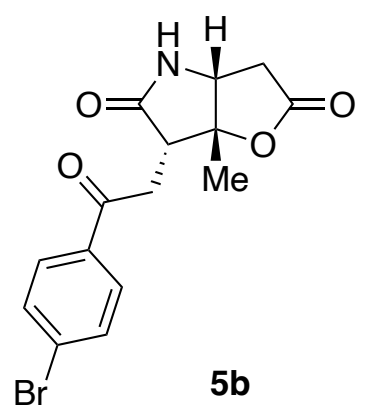

Purified by silica gel chromatography with $\mathrm{CHCl}_{3}$ and EtOAc (gradually 5:1-1:1), yellow solid, 44\% yield (78.3 mg), syn/anti $>20: 1,95 \%$ ee; $[\alpha]^{24}{ }_{\mathrm{D}}=+88.9^{\circ}$ (c $\left.1.00, \mathrm{CHCl}_{3}\right)$; m.p. 87.0-89.0 ${ }^{\circ} \mathrm{C} ;{ }^{1} \mathrm{H} \mathrm{NMR}\left(\mathrm{CDCl}_{3}, 400 \mathrm{MHz}\right): \delta 7.89(\mathrm{~d}, 2 \mathrm{H}, J=8.4 \mathrm{~Hz}), 7.63$ (d, $2 \mathrm{H}, J=8.4 \mathrm{~Hz}), 7.05$ (brs, $1 \mathrm{H}), 4.14$ (d, 1H, $J=5.6 \mathrm{~Hz}), 3.52$ $(\mathrm{dd}, 1 \mathrm{H}, J=3.4,17.8 \mathrm{~Hz}), 3.34-3.46(\mathrm{~m}, 2 \mathrm{H}), 2.89$ (dd, 1H, $J=$ 6.0, $18.4 \mathrm{~Hz}), 2.67(\mathrm{~d}, 1 \mathrm{H}, J=18.0 \mathrm{~Hz}), 1.58(\mathrm{~s}, 3 \mathrm{H}) ;{ }^{13} \mathrm{C}\left\{{ }^{1} \mathrm{H}\right\}$

$\mathrm{NMR}\left(\mathrm{CDCl}_{3}, 100 \mathrm{MHz}\right): \delta 196.4,176.2,173.2,135.1,132.2,129.9,129.0,89.2,57.8$, 45.5, 36.0, 34.3, 22.9; Enantiomeric excess of the product was determined by chiral stationary phase HPLC analysis using a ChiralPak AD-H column (hexane/ $i-\mathrm{PrOH}=80: 20$ at $1.0 \mathrm{~mL} / \mathrm{min}$ ); $\lambda=254 \mathrm{~nm}$; $\mathrm{t}$ major $=18.8 \mathrm{~min}, \mathrm{t}$ minor $=34.5 \mathrm{~min}$; HRMS (ESI) $\mathrm{m} / z$ : $[\mathrm{M}-$ $\mathrm{H}]^{-}$Calcd for $\mathrm{C}_{15} \mathrm{H}_{13} \mathrm{BrNO}_{4} 350.0028$; Found 350.0027. 


\section{X-ray Crystallographic Date}

Table S1. Crystallographic date of $\mathbf{4 b}$ (CCDC2047467).

The single crystal was obtained through vapor diffusion in EtOAc and pentane. The data of compound $\mathbf{4 b}$ were collected using a Bruker SMART APEX II CCD area detector diffractometer at $90 \mathrm{~K}$. Thermal ellipsoids are shown at 50\% probability.<smiles>C[C@]1(C(CC(=O)c2ccc(Br)cc2)C(N)=O)C=CC(=O)O1</smiles>

4b

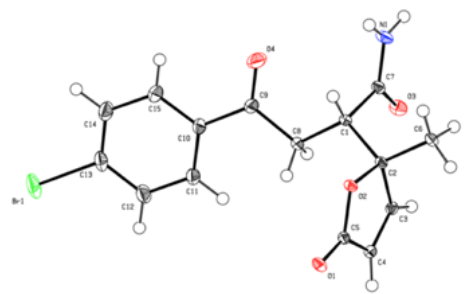

Wavelength $=0.71073$

Bond precision:

$\mathrm{C}-\mathrm{C}=0.0026 \AA$

Cell:

$$
\begin{array}{ll}
\mathrm{a}=9.4170(3) & \mathrm{b}=5.1483(2) \quad \mathrm{c}=14.9447(4) \\
\text { alpha }=90 & \text { beta }=96.707(1) \text { gamma }=90
\end{array}
$$

Temperature: $\quad 90 \mathrm{~K}$

$\begin{array}{lll} & \text { Calculated } & \text { Reported } \\ \text { Volume } & 719.58(4) & 719.58(4) \\ \text { Space group } & \text { P 21 } & \text { P 1 21 1 } \\ \text { Hall group } & \text { P 2yb } & \text { P 2yb } \\ \text { Moiety formula } & \text { C15 H14 Br N O4 } & \text { C15 H14 Br N O4 } \\ \text { Sum formula } & \text { C15 H14 Br N O4 } & \text { C15 H14 Br N O4 } \\ \text { Mr } & 352.17 & 352.18 \\ \text { Dx, g cm-3 } & 1.625 & 1.625 \\ \text { Z } & 2 & 2 \\ \text { Mu (mm-1) } & 2.871 & 2.871 \\ \text { F000 } & 356.0 & 356.0 \\ \text { F000' } & 355.60 & \\ \text { h, k, lmax } & 13,7,21 & 13,7,21 \\ \text { Nref } & 4406[2428] & 4384 \\ \text { Tmin, Tmax } & 0.743,0.823 & 0.625,0.868 \\ \text { Tmin' } & 0.342 & \end{array}$

Correction method $=\#$ Reported T Limits: $\mathrm{Tmin}=0.625 \mathrm{Tmax}=0.868$

AbsCorr $=$ NUMERICAL

Data completeness $=1.81 / 1.00$

Theta $(\max )=30.503$

$\mathrm{R}($ reflections $)=0.0197$ ( 4213) $\mathrm{wR} 2($ reflections $)=0.0499(4384)$

$\mathrm{S}=1.067$ Npar $=191$ 
Table S2. Crystallographic date of $\mathbf{5 b}$ (CCDC2047468).

The single crystal was obtained through vapor diffusion in diethyl ether and hexane. The data of compound $\mathbf{5 b}$ were collected using a Bruker SMART APEX II CCD area detector diffractometer at $90 \mathrm{~K}$. Thermal ellipsoids are shown at 50\% probability.
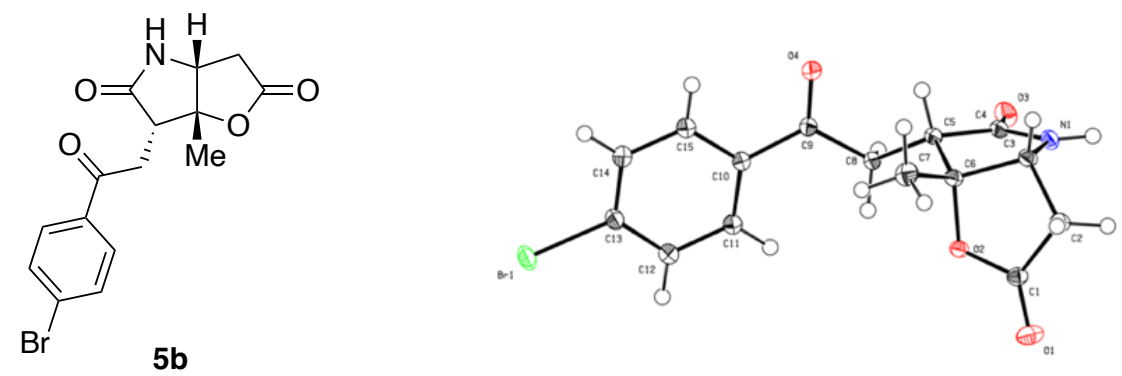

Bond precision: $\mathrm{C}-\mathrm{C}=0.0033 \AA$

Wavelength $=0.71073$

Cell:

$$
\begin{array}{lll}
\mathrm{a}=5.5952(2) & \mathrm{b}=8.2745(2) & \mathrm{c}=31.2751(9) \\
\text { alpha }=90 & \text { beta }=90 & \text { gamma }=90
\end{array}
$$

Temperature: $\quad 90 \mathrm{~K}$

$\begin{array}{lll} & \text { Calculated } & \text { Reported } \\ \text { Volume } & 1447.96(8) & 1447.96(8) \\ \text { Space group } & \text { P 21 21 21 } & \text { P 21 21 21 } \\ \text { Hall group } & \text { P 2ac 2ab } & \text { P 2ac 2ab } \\ \text { Moiety formula } & \text { C15 H14 Br N O4 } & \text { C15 H14 Br N O4 } \\ \text { Sum formula } & \text { C15 H14 Br N O4 } & \text { C15 H14 Br N O4 } \\ \text { Mr } & 352.17 & 352.18 \\ \text { Dx, g cm-3 } & 1.615 & 1.616 \\ \text { Z } & 4 & 4 \\ \text { Mu (mm-1) } & 2.854 & 2.854 \\ \text { F000 } & 712.0 & 712.0 \\ \text { F000' } & 711.19 & \\ \text { h, k, lmax } & 8,11,44 & 8,11,44 \\ \text { Nref } & 4421[2577] & 4405 \\ \text { Tmin, Tmax } & 0.444,0.644 & 0.568,0.762 \\ \text { Tmin' } & 0.415 & \end{array}$

Correction method $=\#$ Reported T Limits: $\mathrm{Tmin}=0.568 \mathrm{Tmax}=0.762$

AbsCorr $=$ NUMERICAL

Data completeness $=1.71 / 1.00$

$\operatorname{Theta}(\max )=30.571$

$\mathrm{R}($ reflections $)=0.0266(4126)$

$$
\text { wR2(reflections) }=0.0647(4405)
$$

$\mathrm{S}=1.069$

Npar $=191$ 


\section{Procedure for Glam Scale Experiment}

$\alpha$-Angelica lactone (2a, $698.8 \mu \mathrm{L}, 7.8 \mathrm{mmol}, 1.2$ equiv) was added to a solution of $\beta$ cyanoenone (1a, $1.021 \mathrm{~g}, 6.5 \mathrm{mmol})$ and organocatalyst $\mathbf{G}(123.8 \mathrm{mg}, 0.2 \mathrm{mmol}, 3 \mathrm{~mol} \%$ ) in dry toluene $(6.5 \mathrm{~mL})$ at $\mathrm{rt}$ After stirring at $\mathrm{rt}$ for $1 \mathrm{~h}$, the reaction mixture was purified by silica gel chromatography with hexane and EtOAc (gradually 10:1-3:1) to afford the pure compound 3a as a white solid (1.581 g, 97\% yield, syn:anti $>20: 1,98 \%$ ee).

\section{References}

1) Sakai, T.; Hirashima, S.; Yamashita, Y.; Arai, R.; Nakashima, K.; Yoshida, A.; Koseki, Y.; Miura, T. J. Org. Chem. 2017, 82, 4661.

2) Kong, L.-P.; Li, N.-K.; Zhang, S.-Y.; Chen, X.; Zhao, M.; Zhang, Y.-F.; Wang, X.-W. Org. Biomol. Chem. 2014, 12, 8656.

3) Choudhury, A. R.; Manna, M. S.; Mukherjee. S. Chem. Sci., 2017, 8, 6686.

4) Hirashima, S.; Nakashima, K.; Fujino, Y.; Arai, R.; Sakai, T.; Kawada, M.; Koseki, Y.; Murahashi, M.; Tada, N.; Itoh, A.; Miura, T. Tetrahedron Lett. 2014, 55, 4619.

5) Kanada, Y.; Yuasa, H.; Nakashima, K.; Murahashi, M.; Tada, N.; Itoh, A. Koseki, Y.; Miura, T. Tetrahedron Lett. 2013, 54, 4896.

6) Thies, T.; Watanabe, M. Molbank 2003, M334.

7) Hung, C.-H.; Gandeepan, P.; Cheng, L.-C.; Chen, L.-C.; Cheng, M.-J.; Cheng, C.-H. J. Am. Chem. Soc. 2017, 139, 17015.

8) Wu, Y.; Singh, R. P.; Deng, L. J. Am. Chem. Soc. 2011, 133, 12458.

9) Manna, M. S.; Kumar, V.; Mukherjee, S. Chem. Commun. 48, 5193. 
11. NMR Spectra

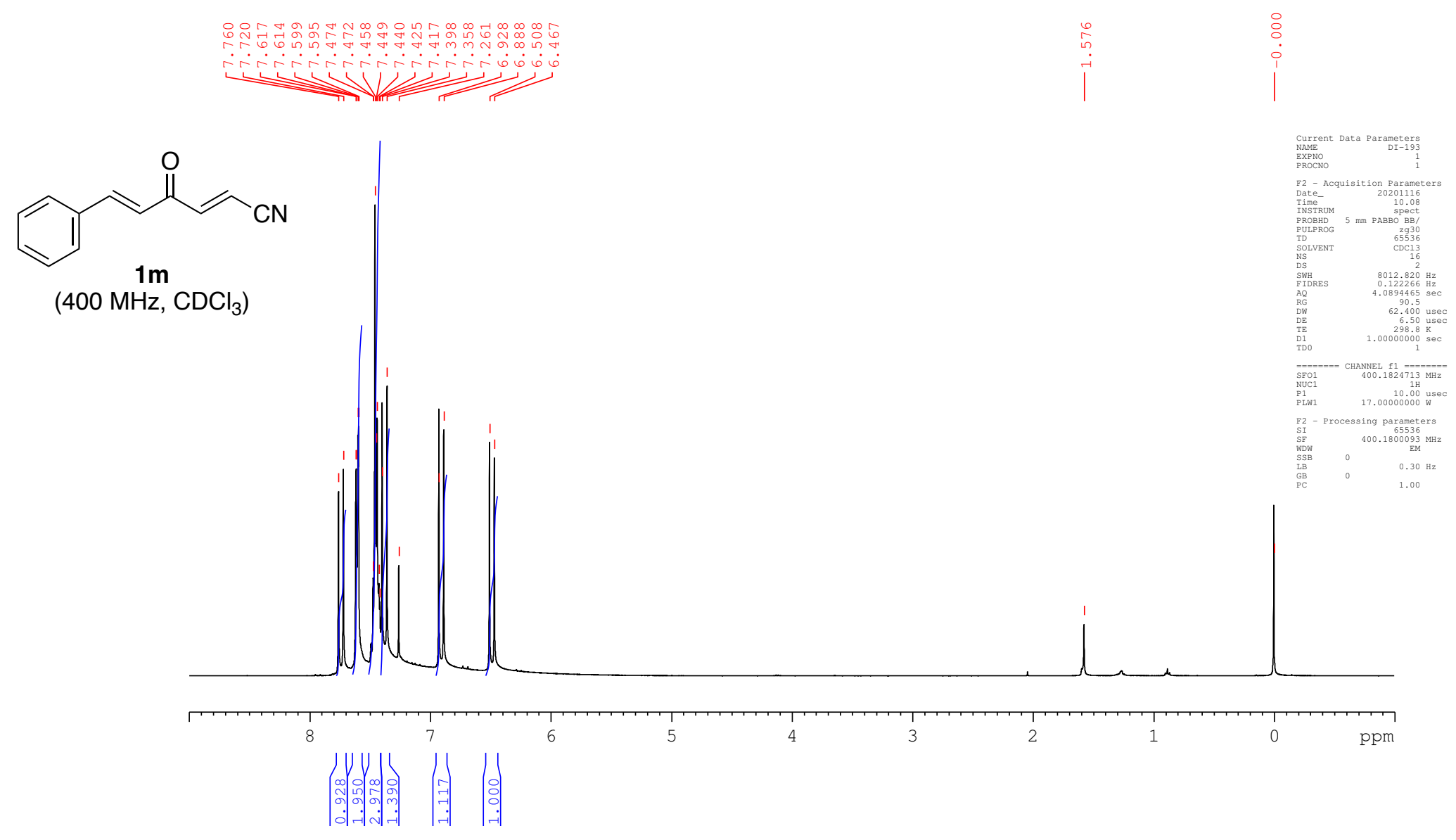




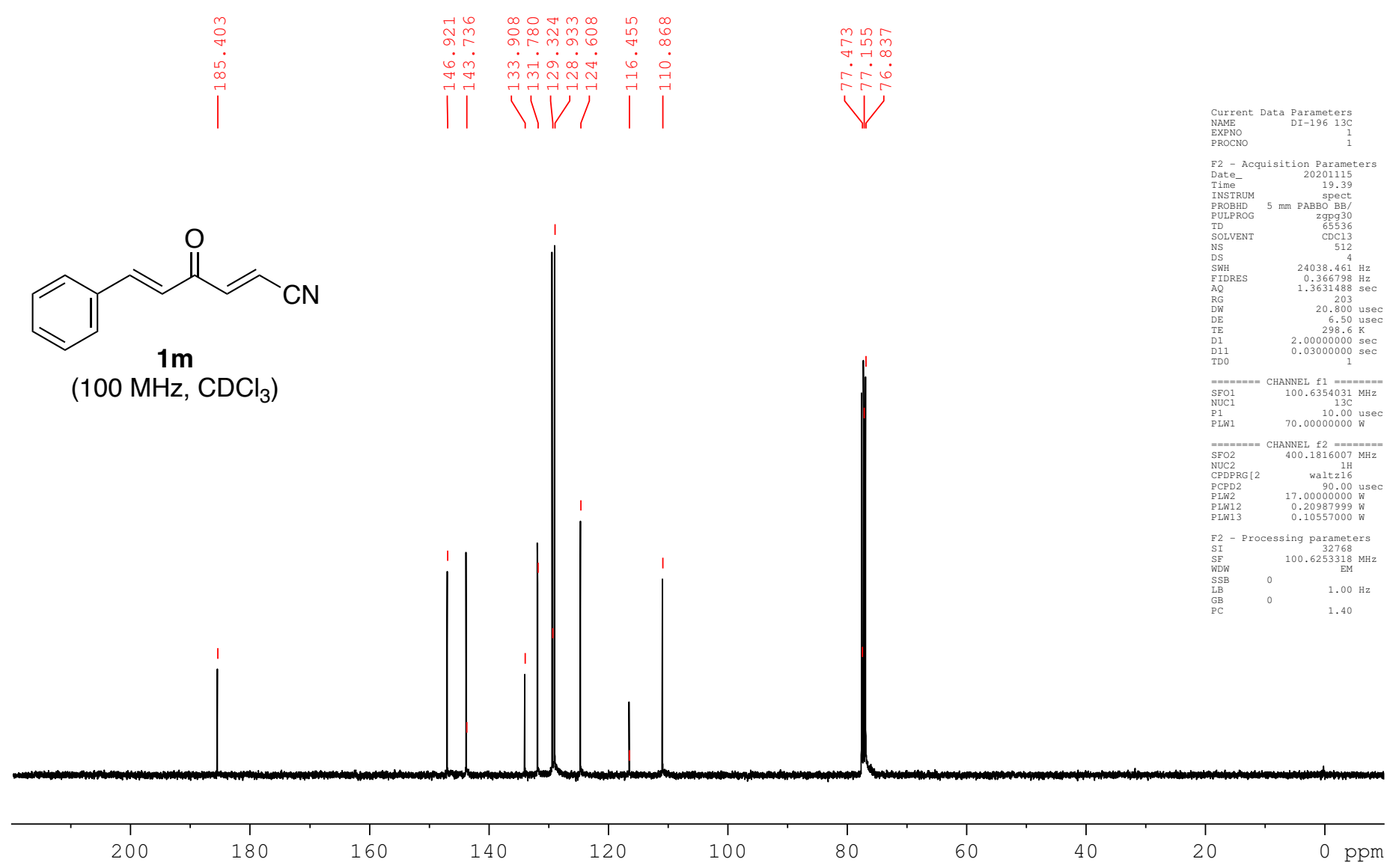




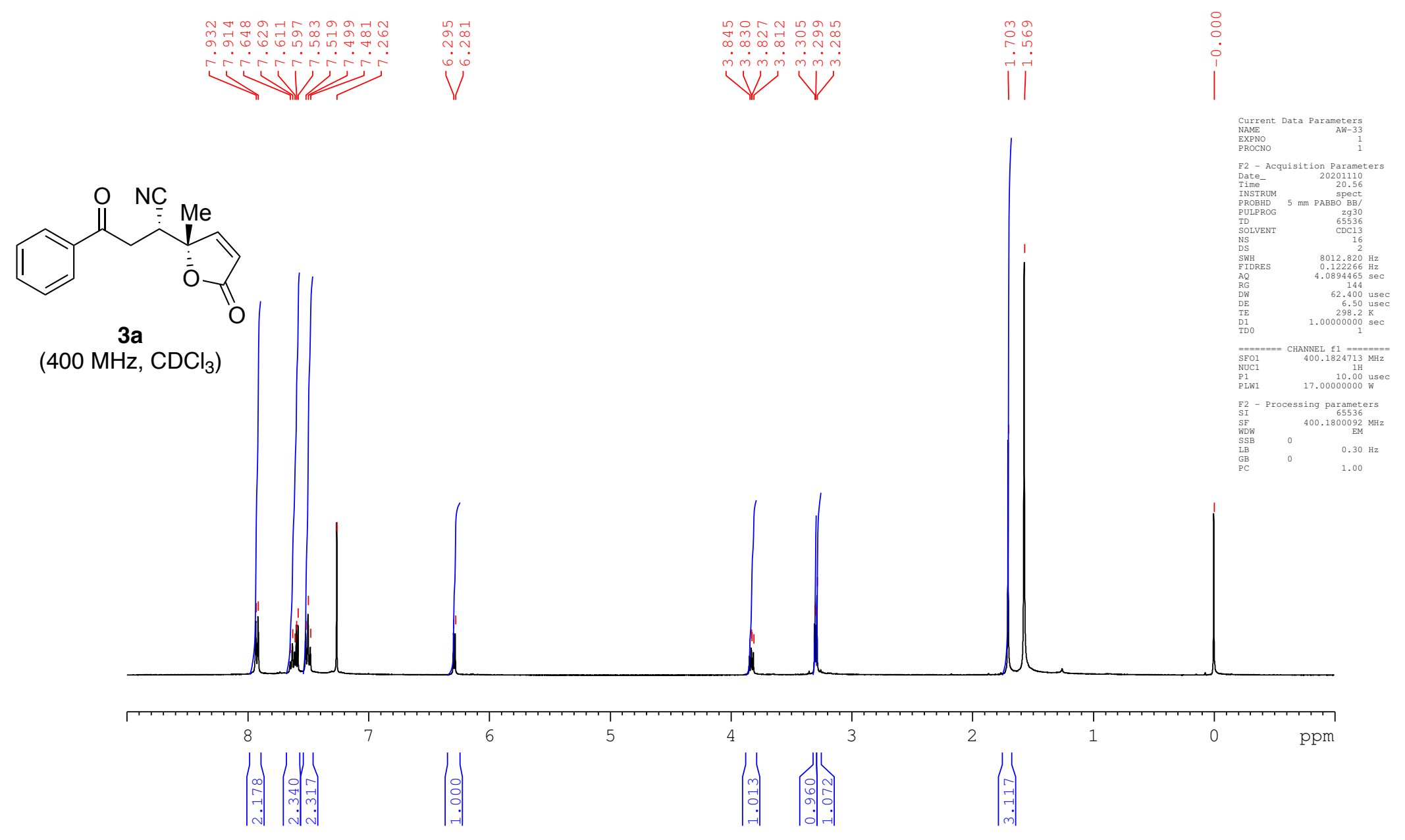




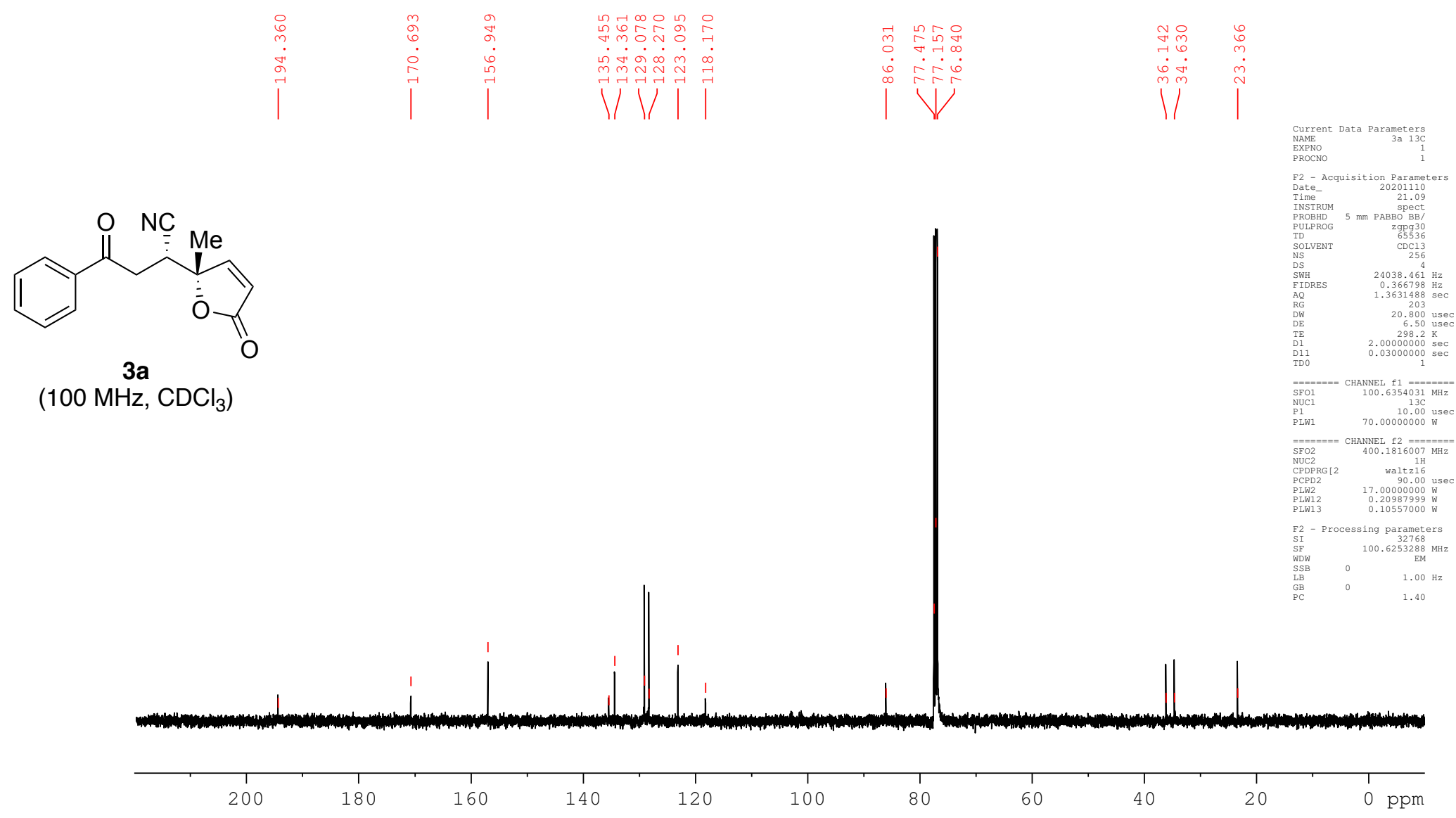




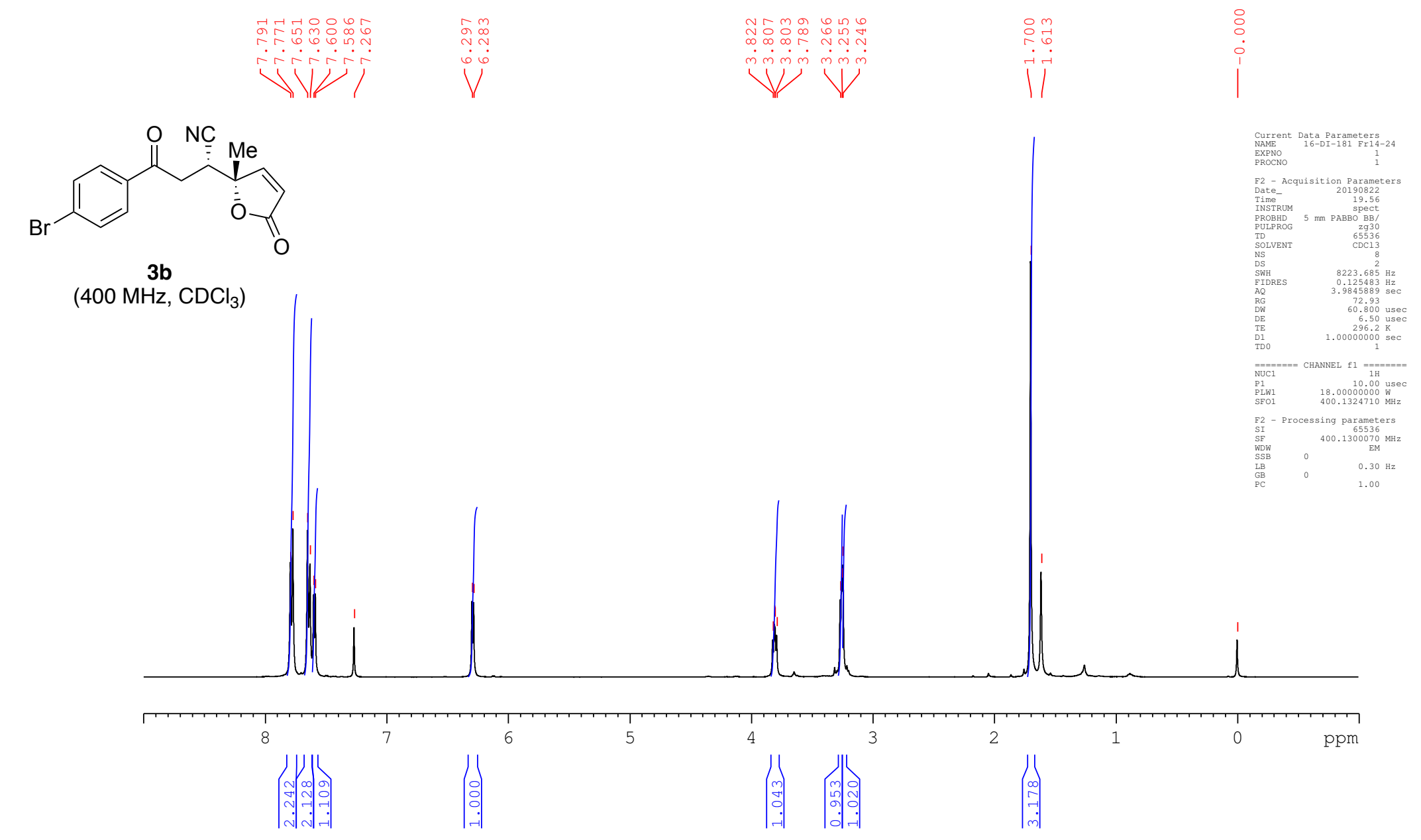




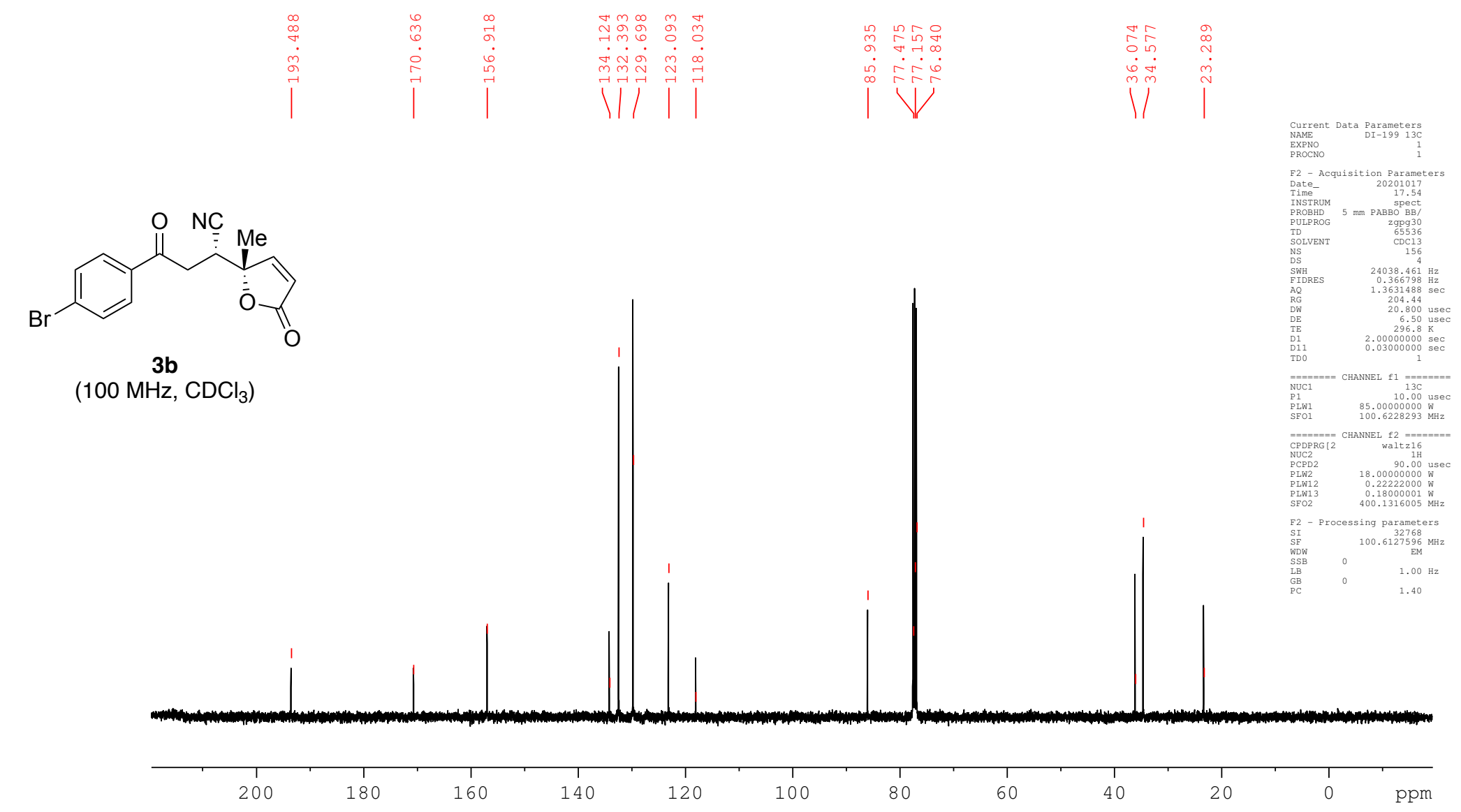




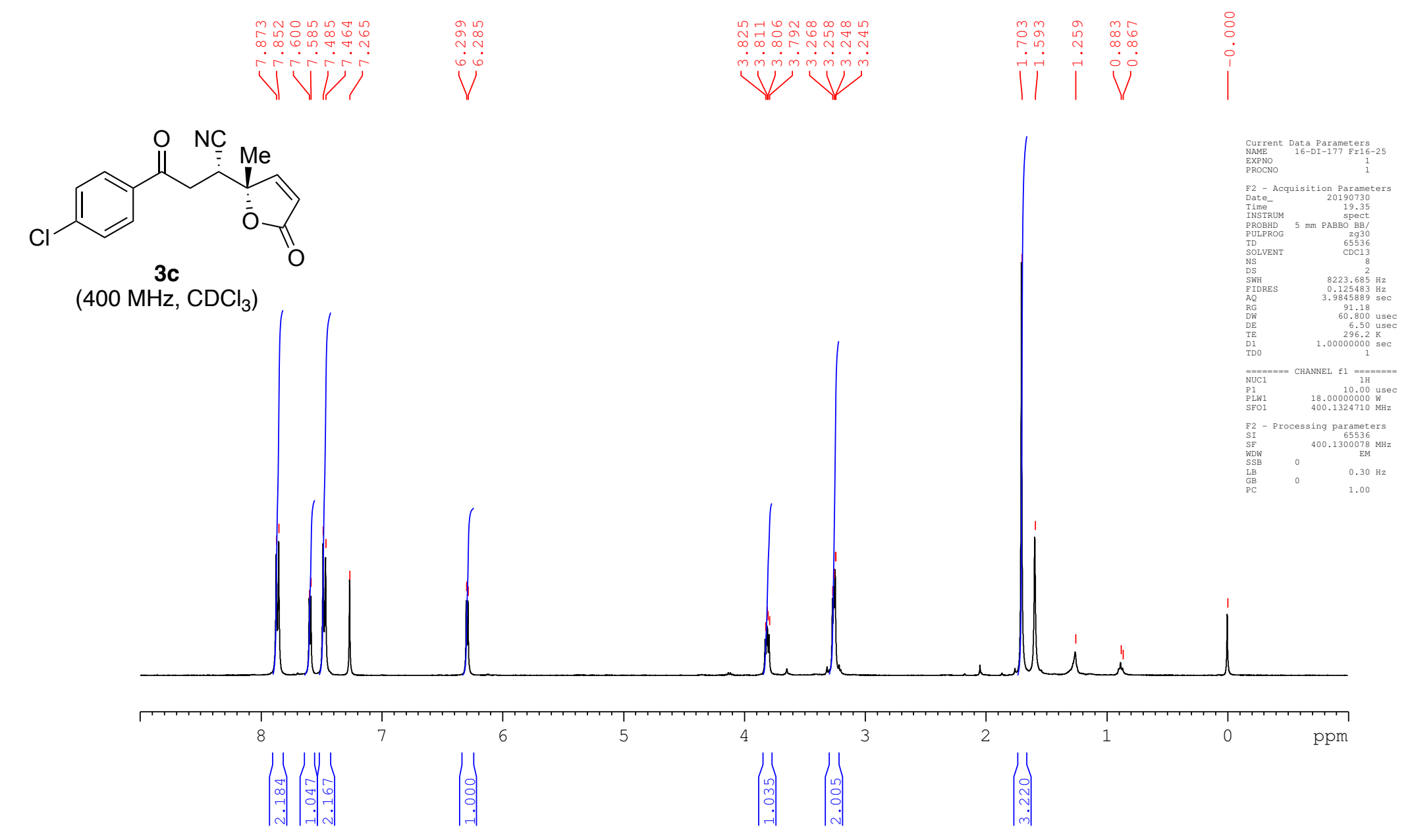




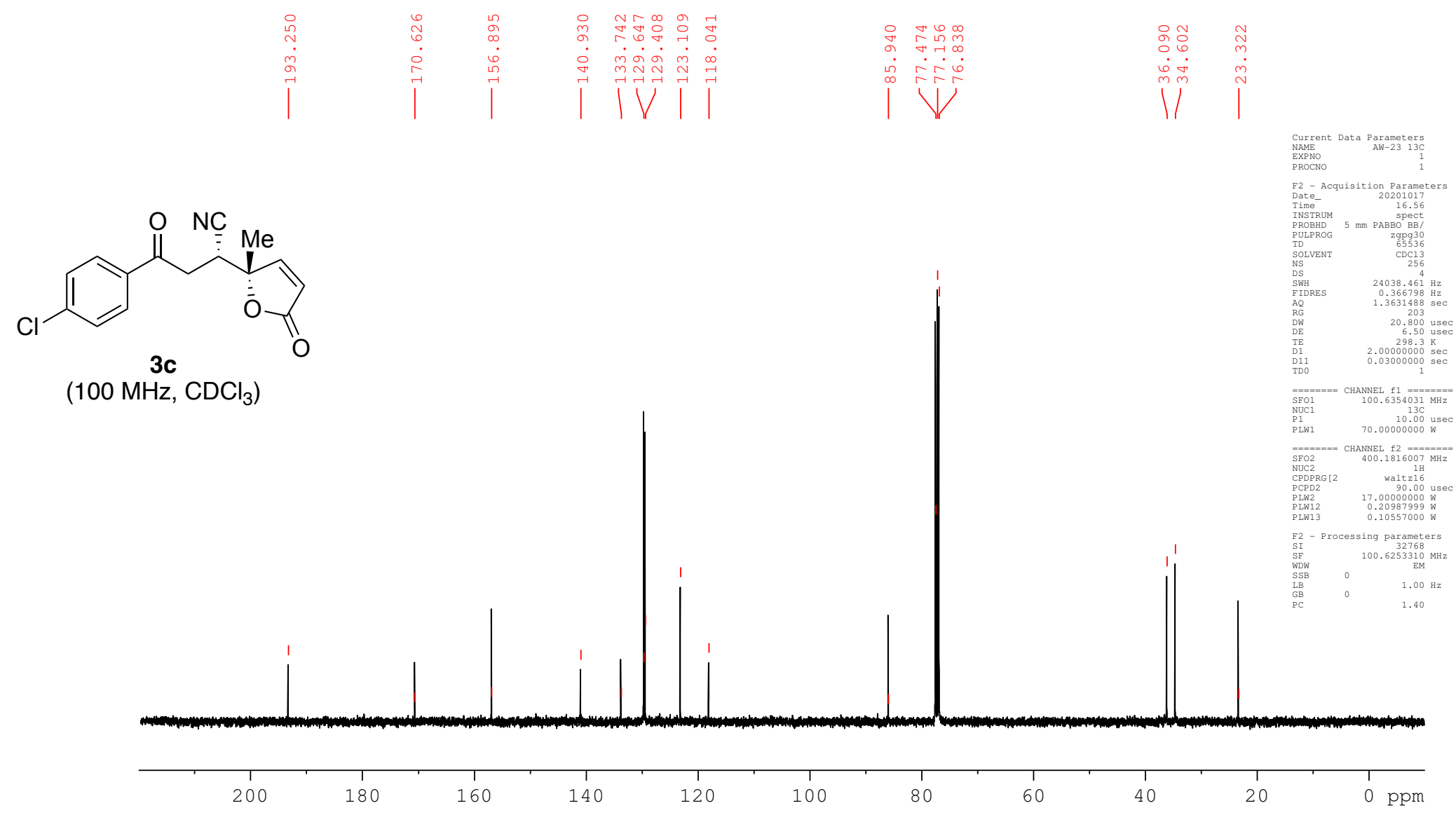




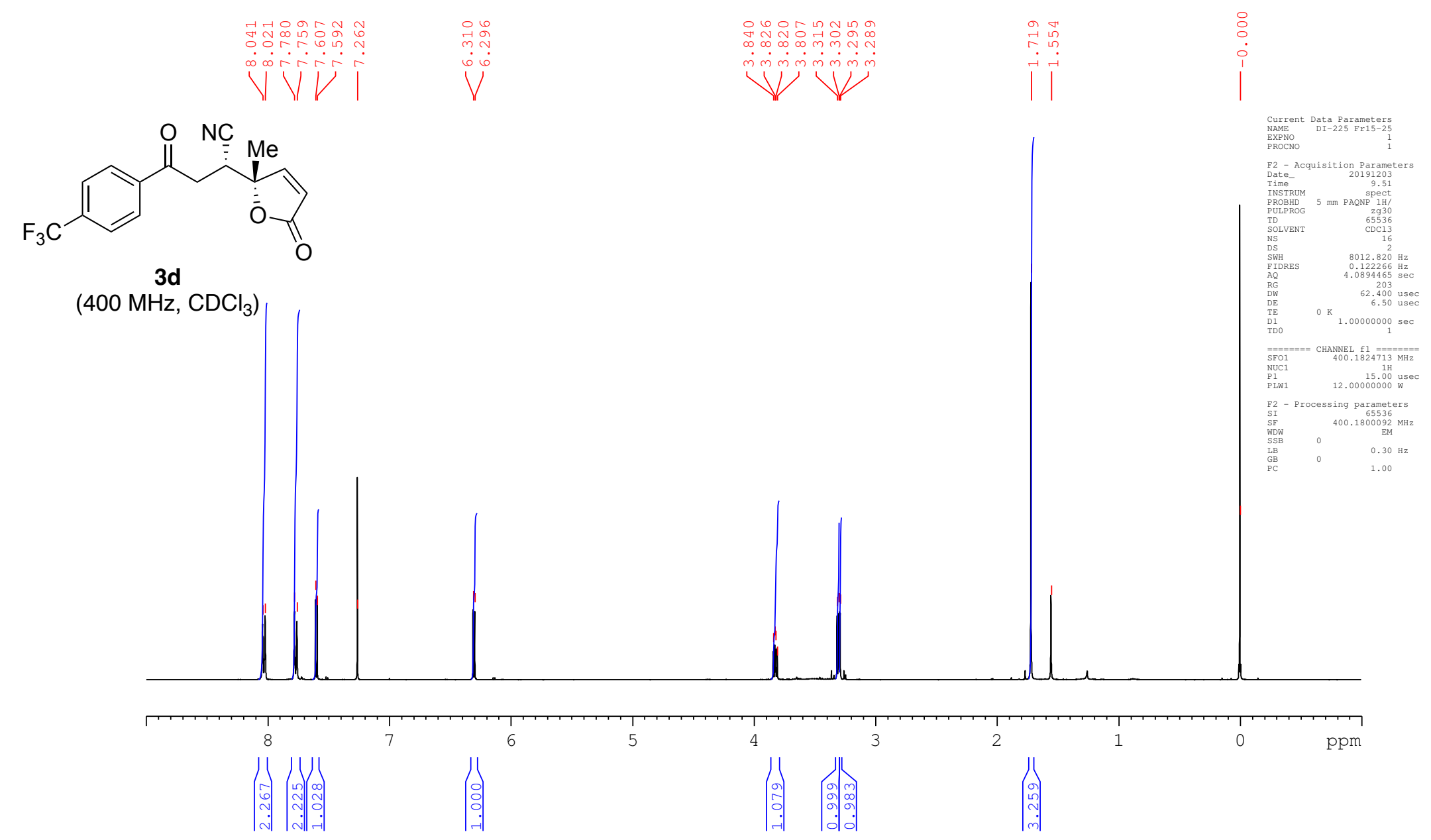




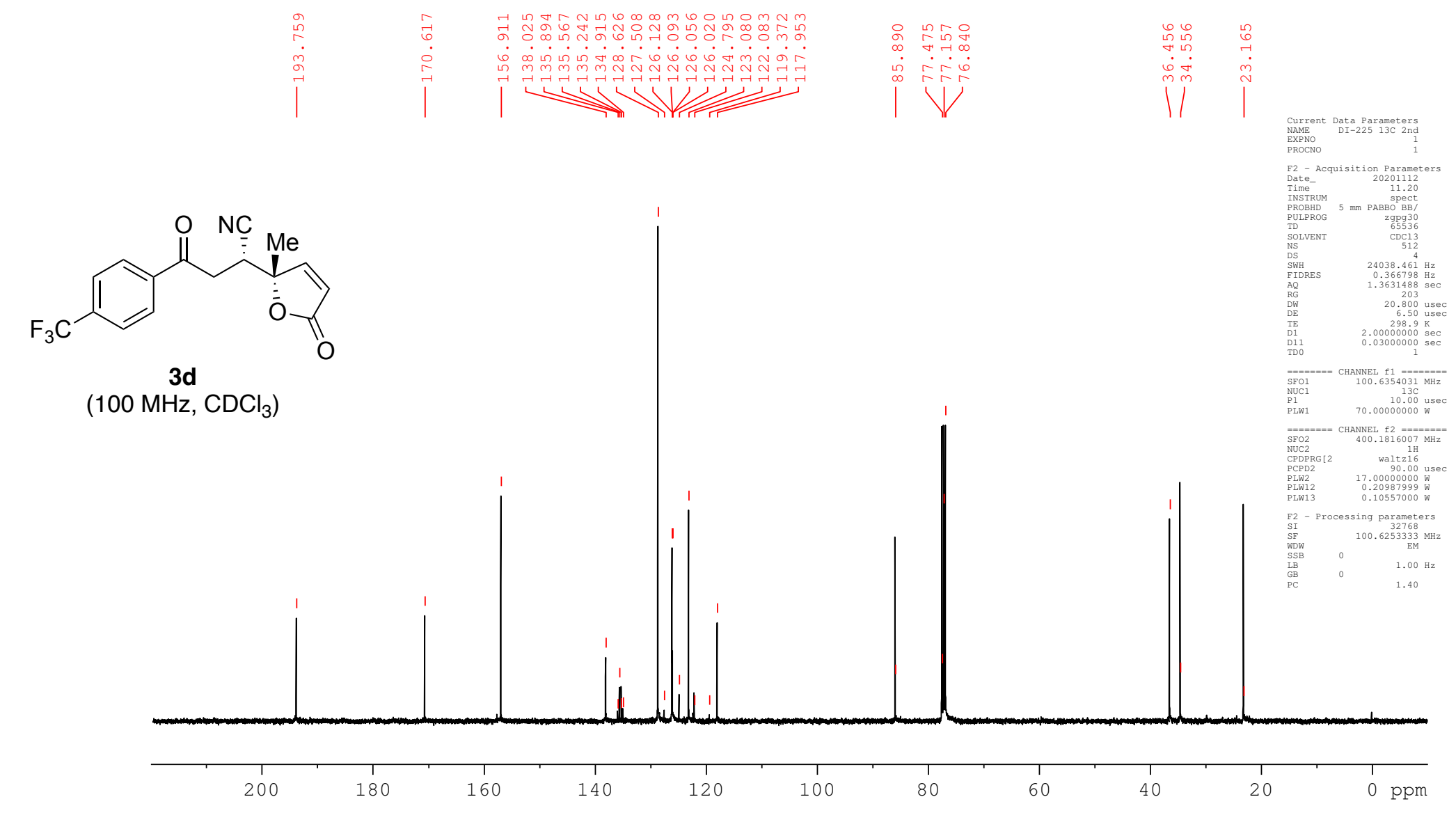




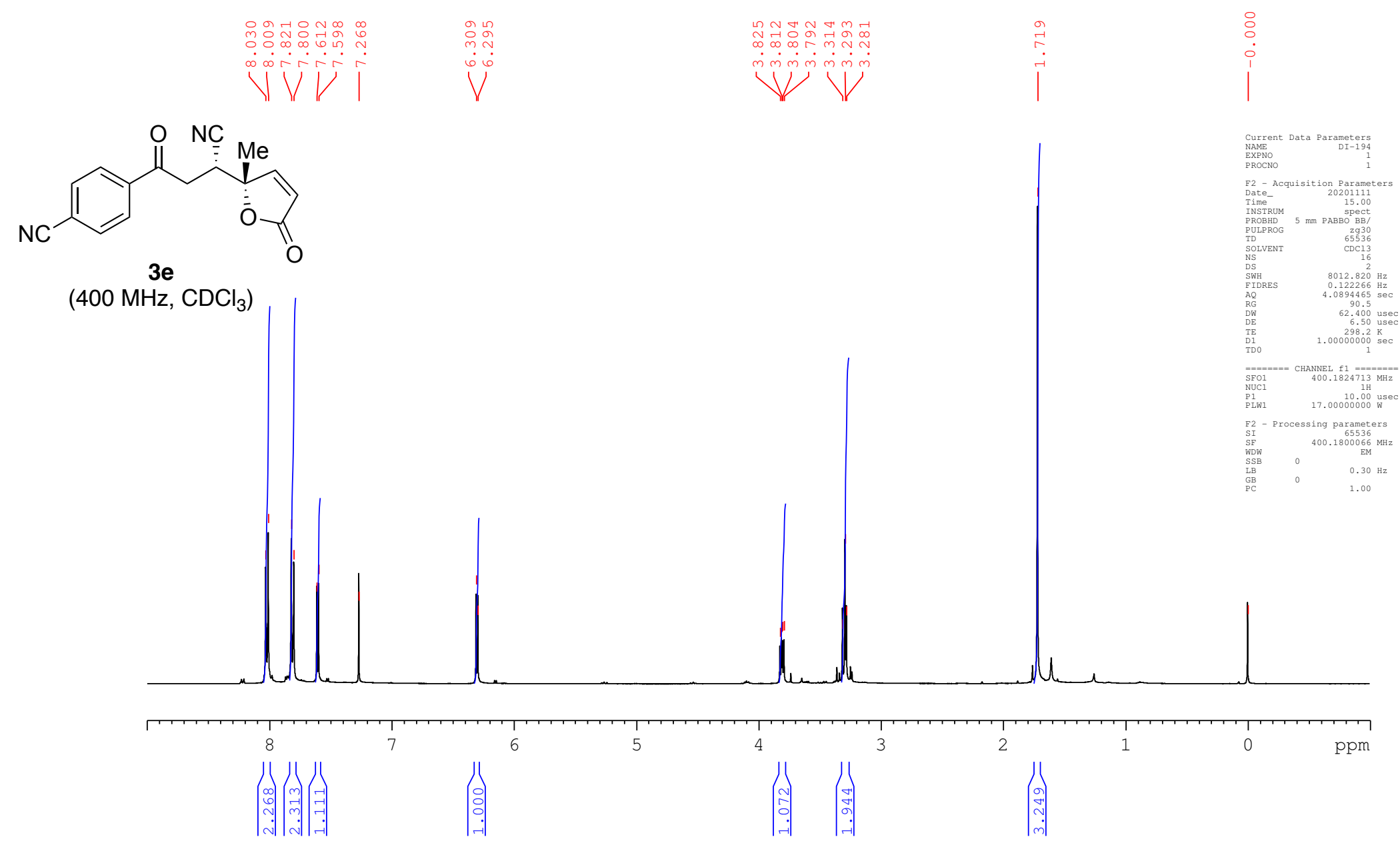




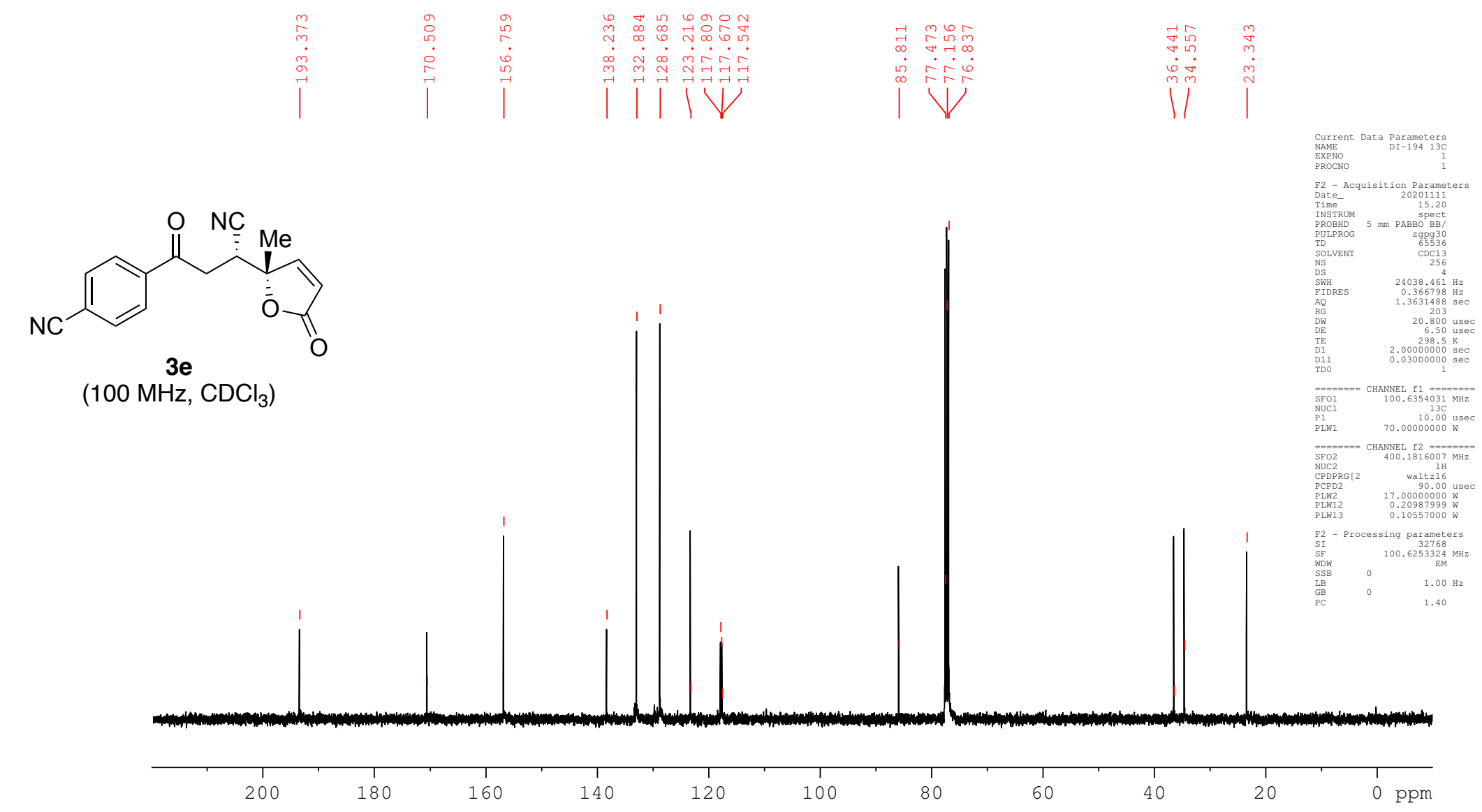




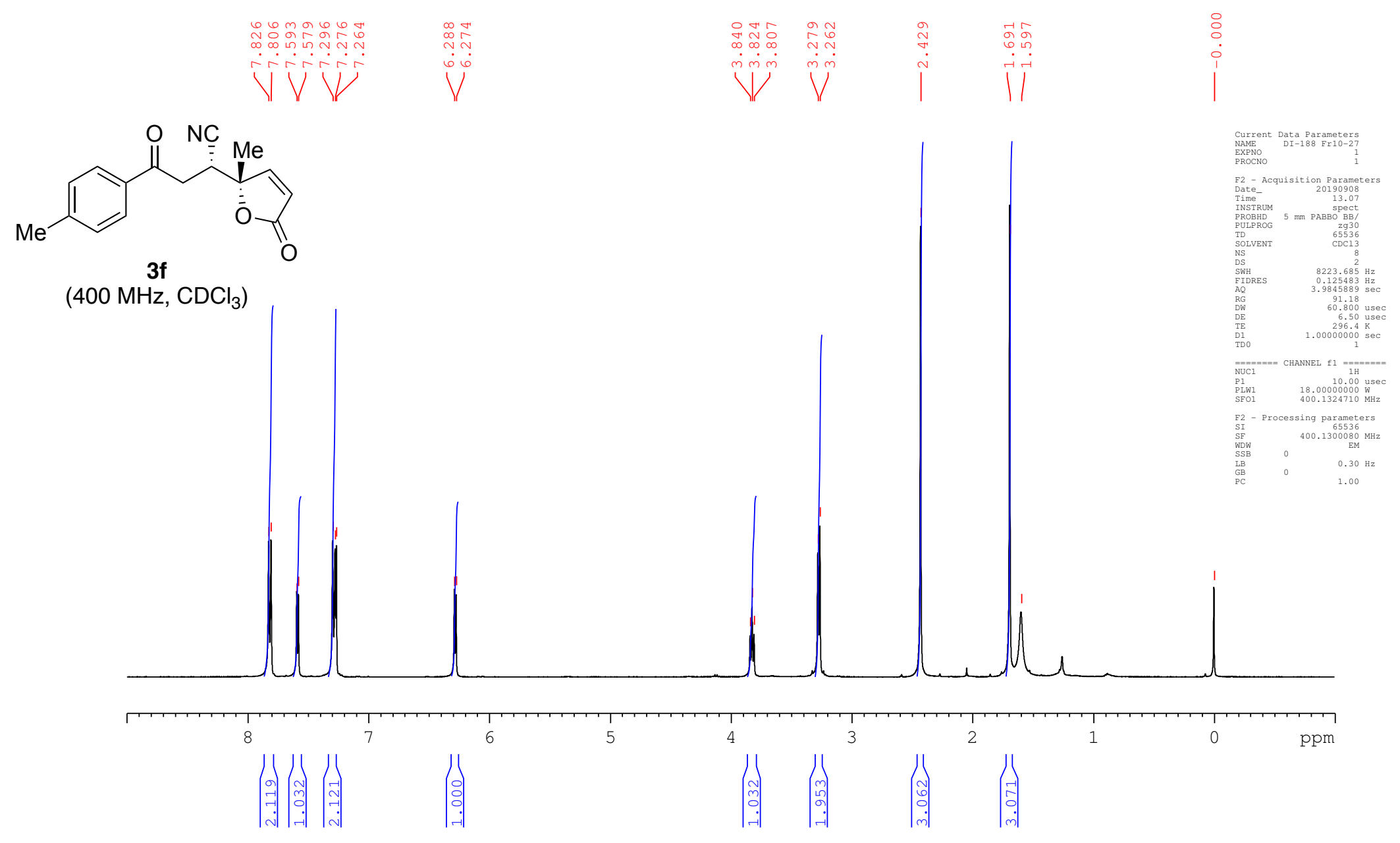




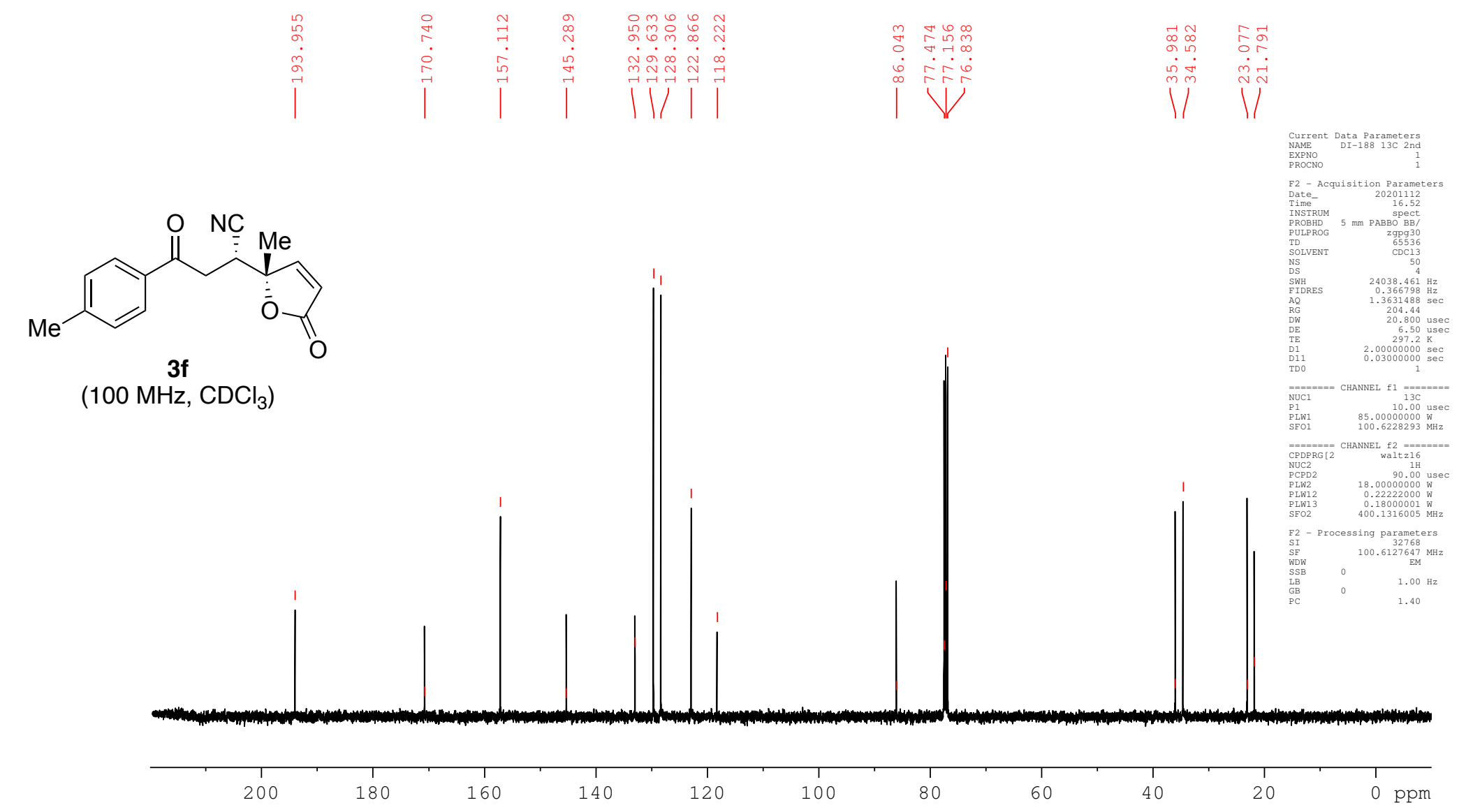




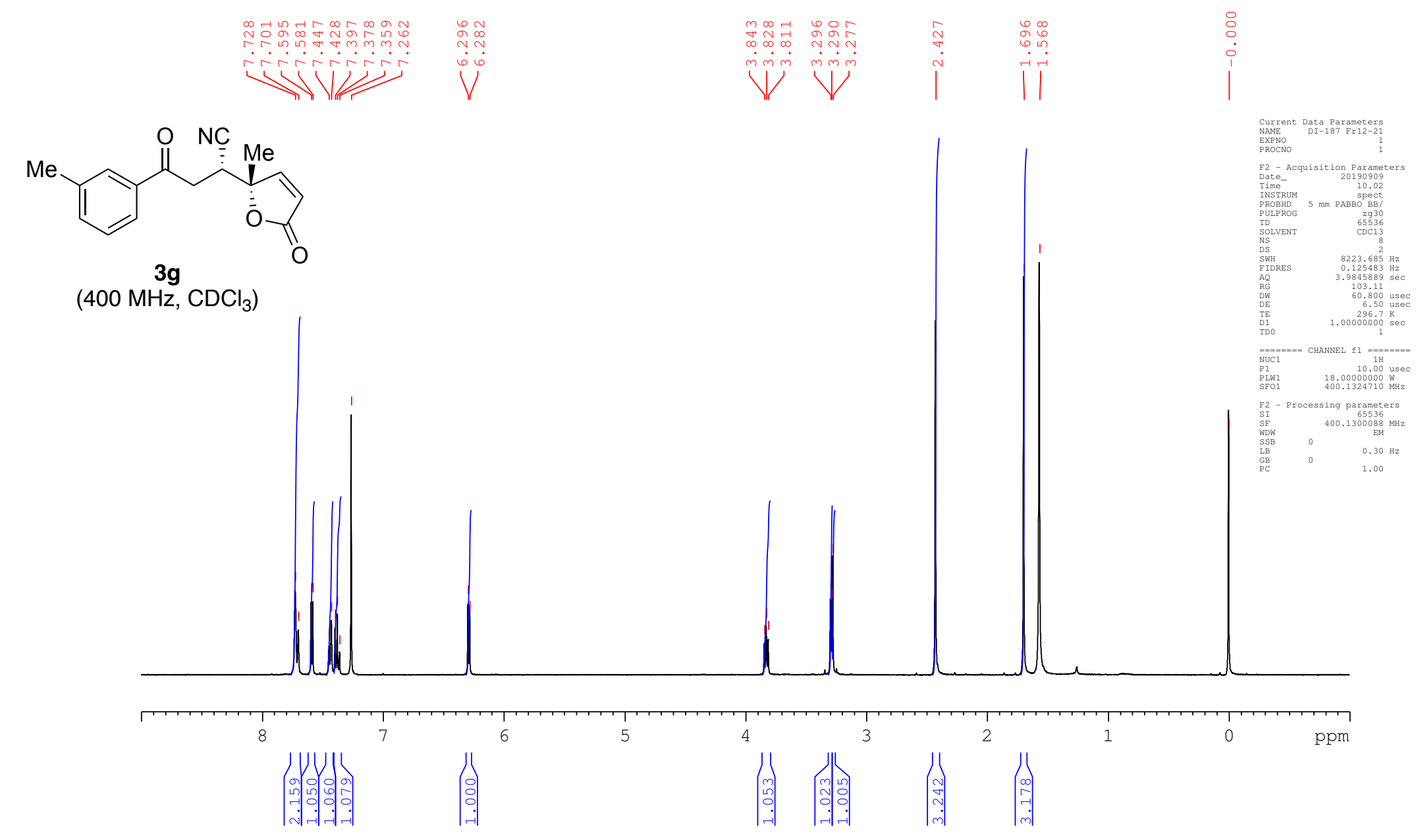




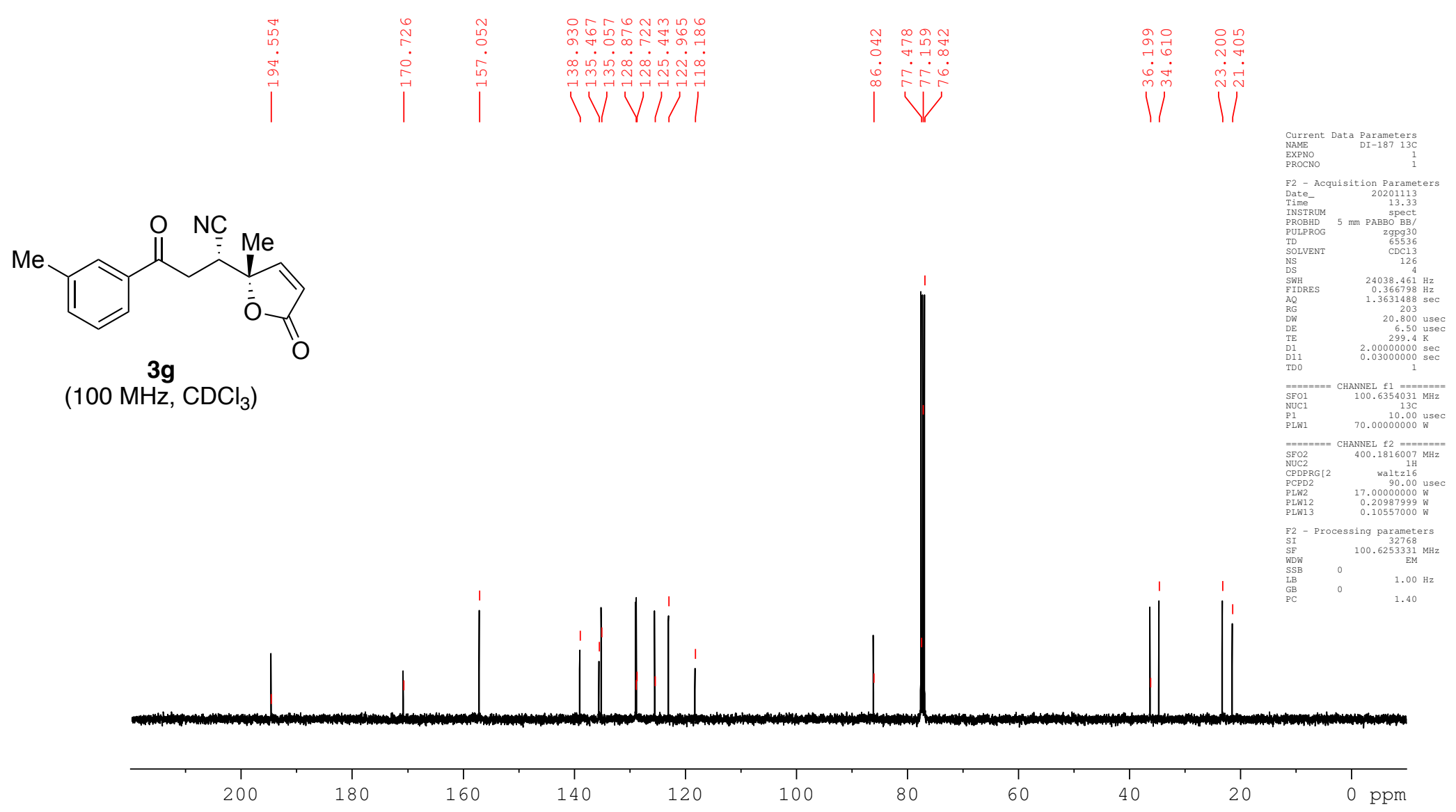




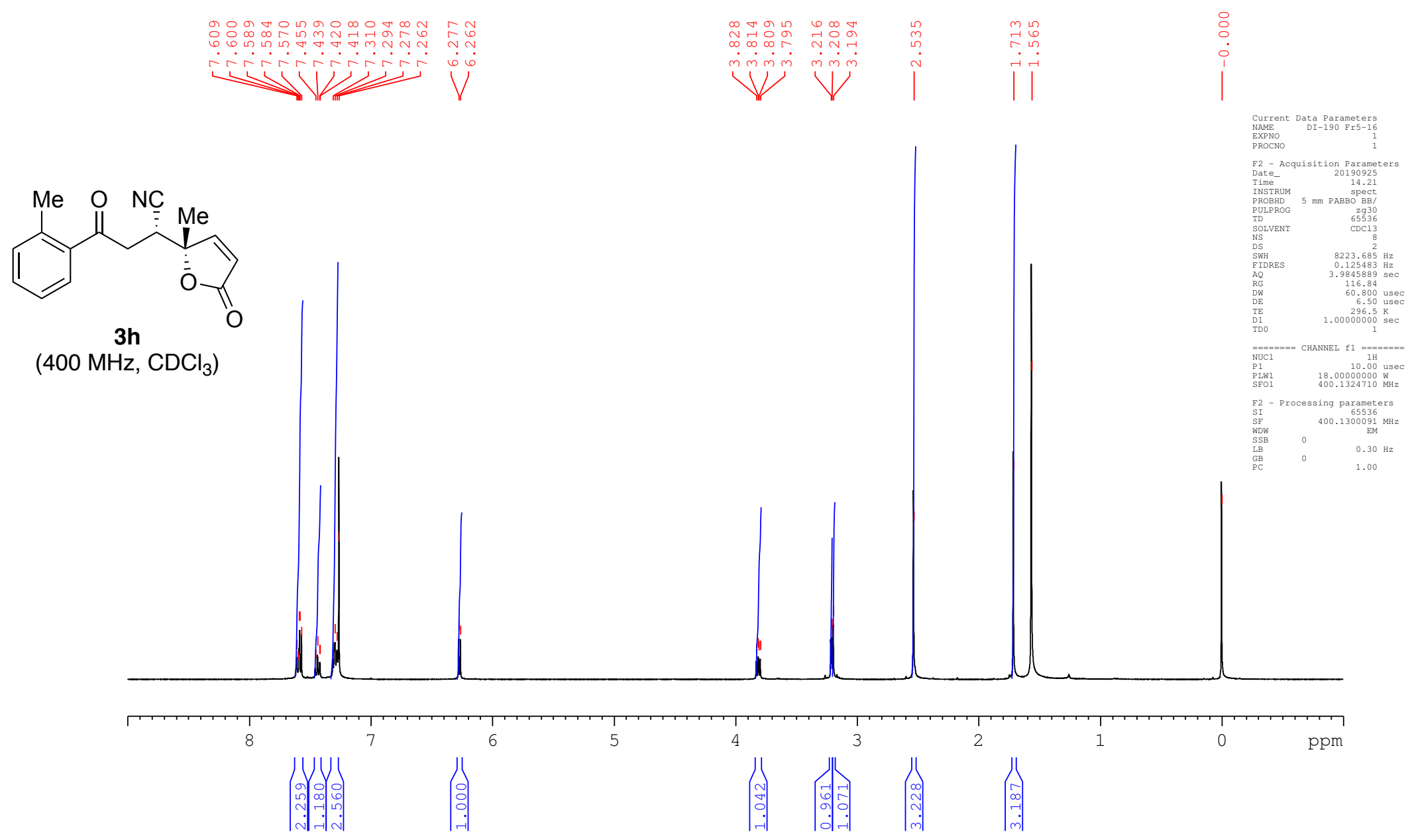




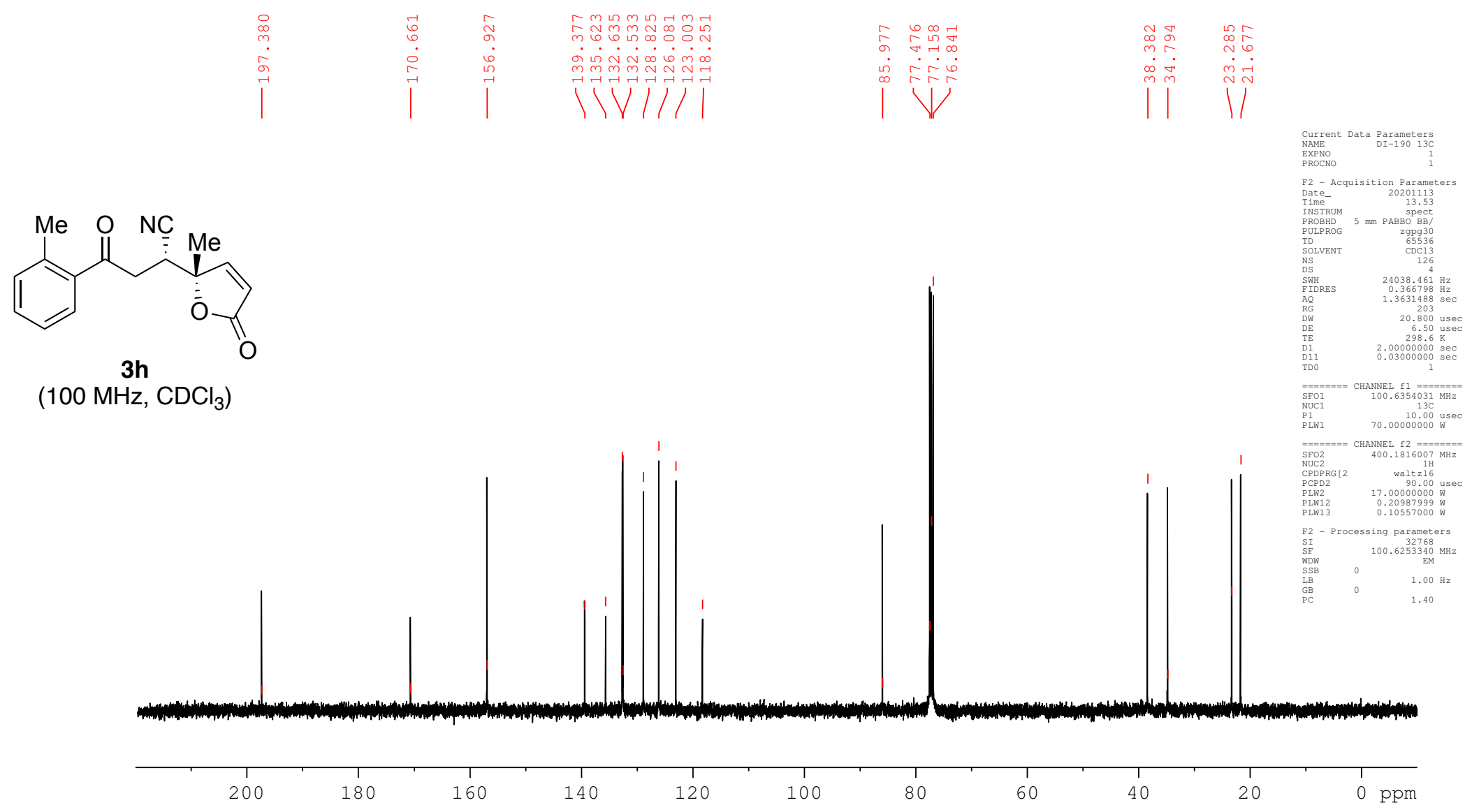




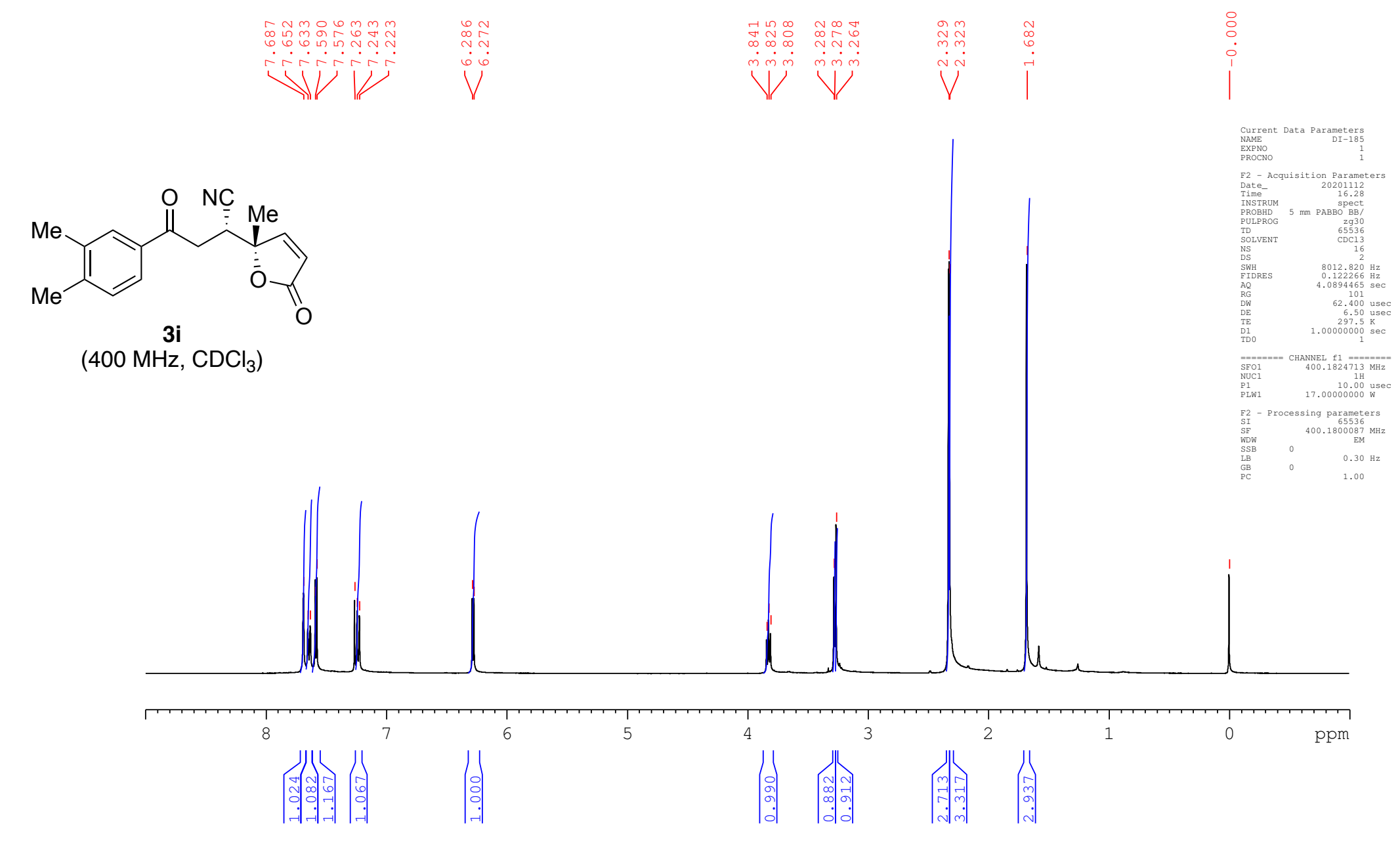




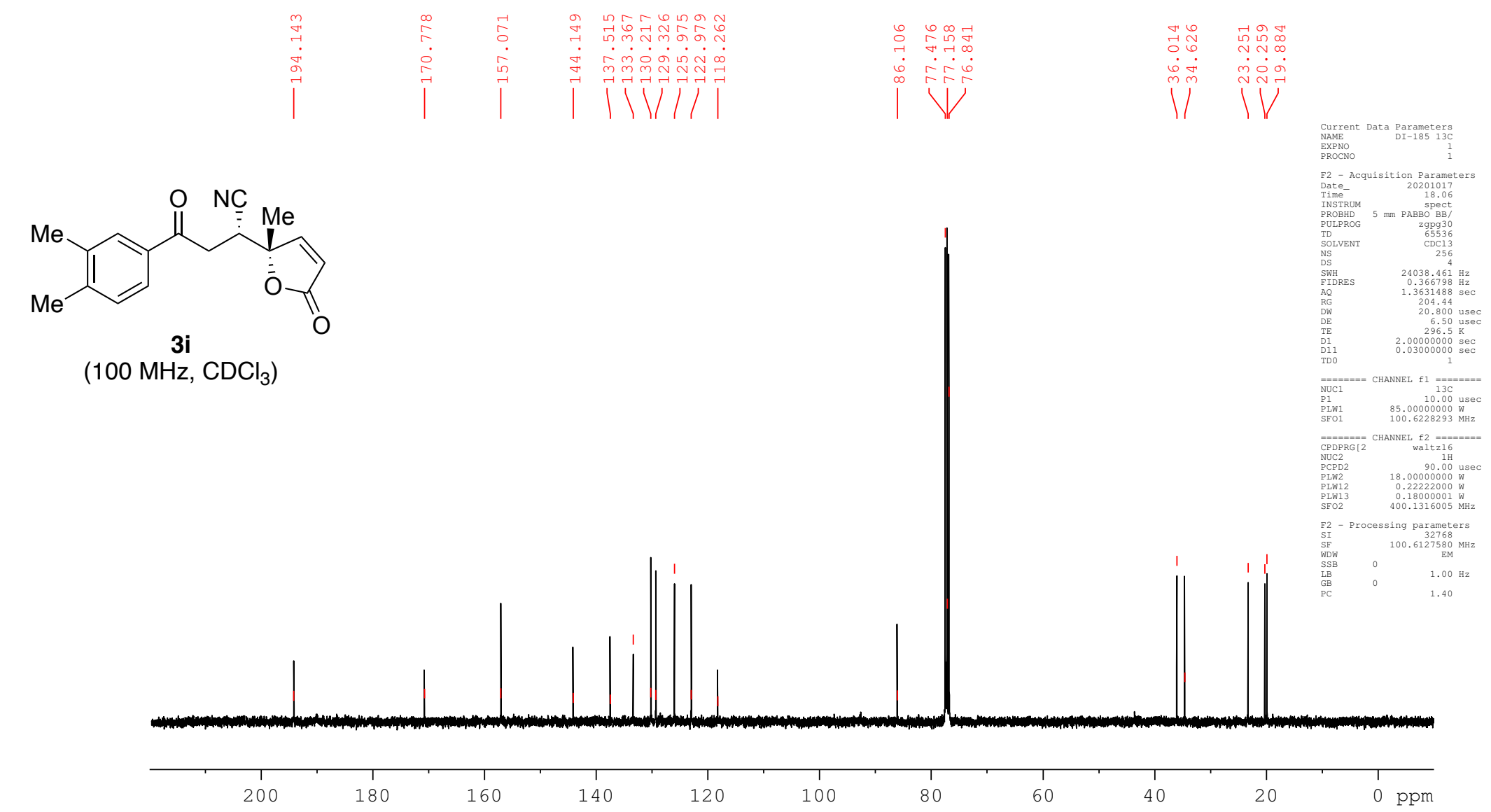




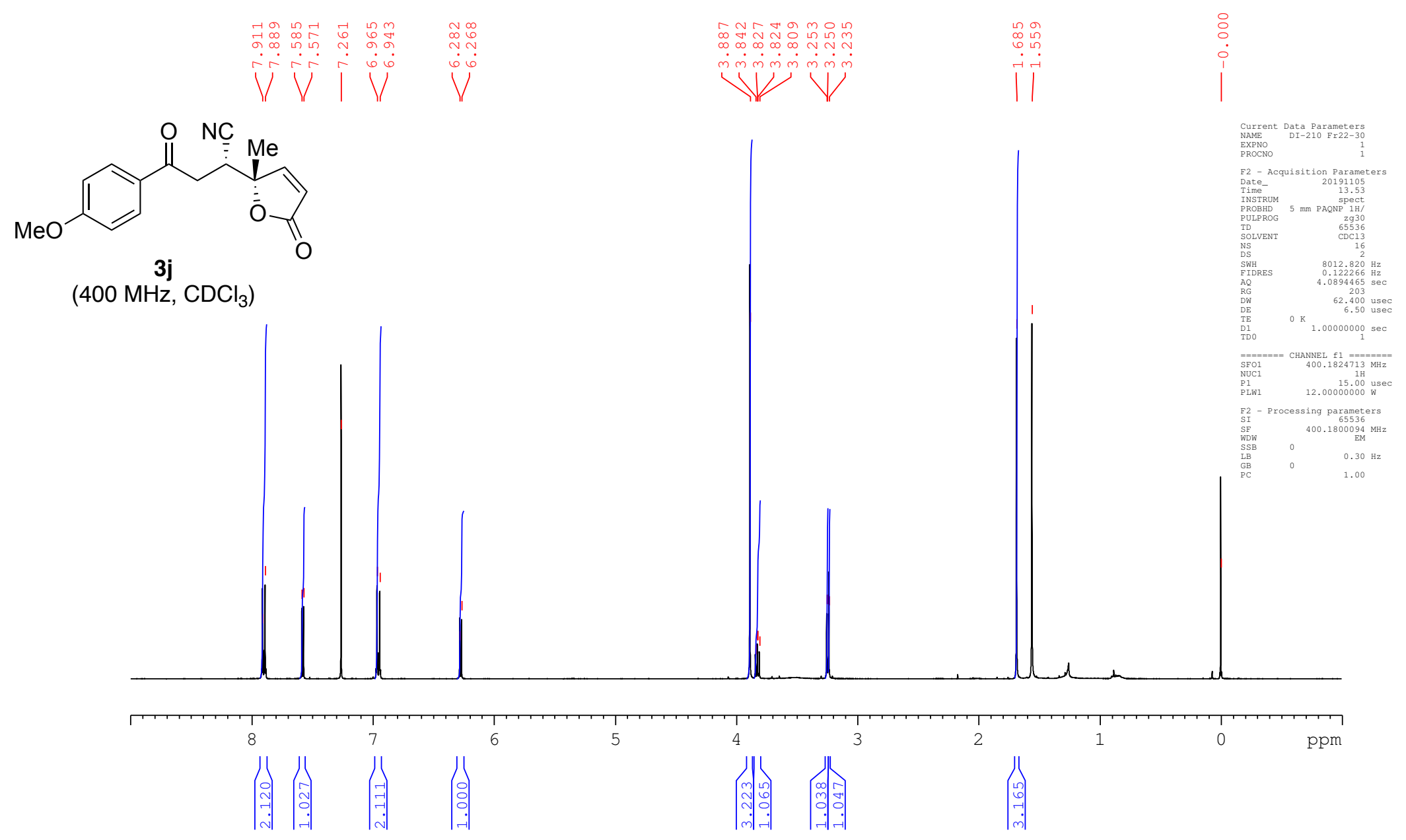




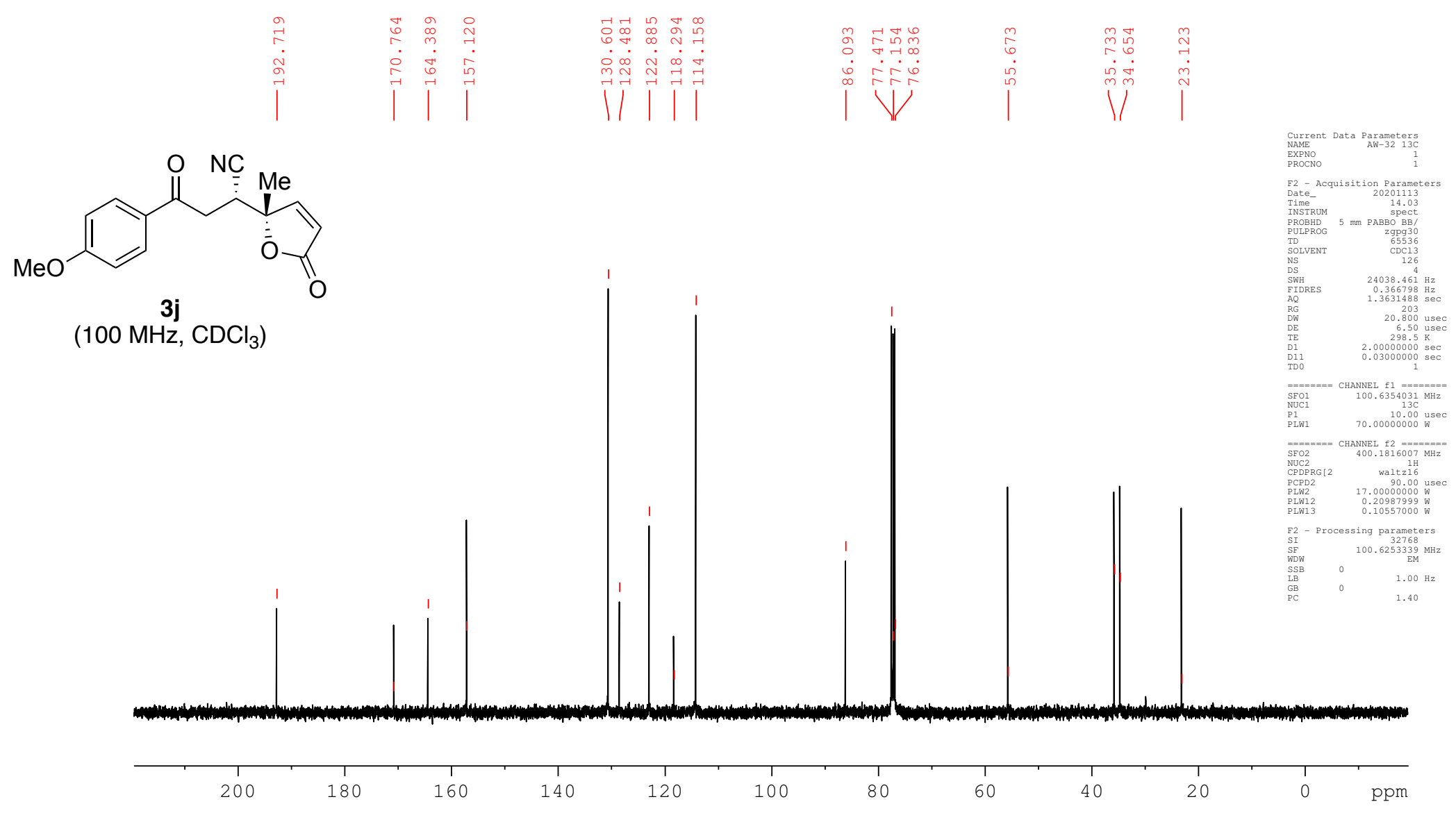




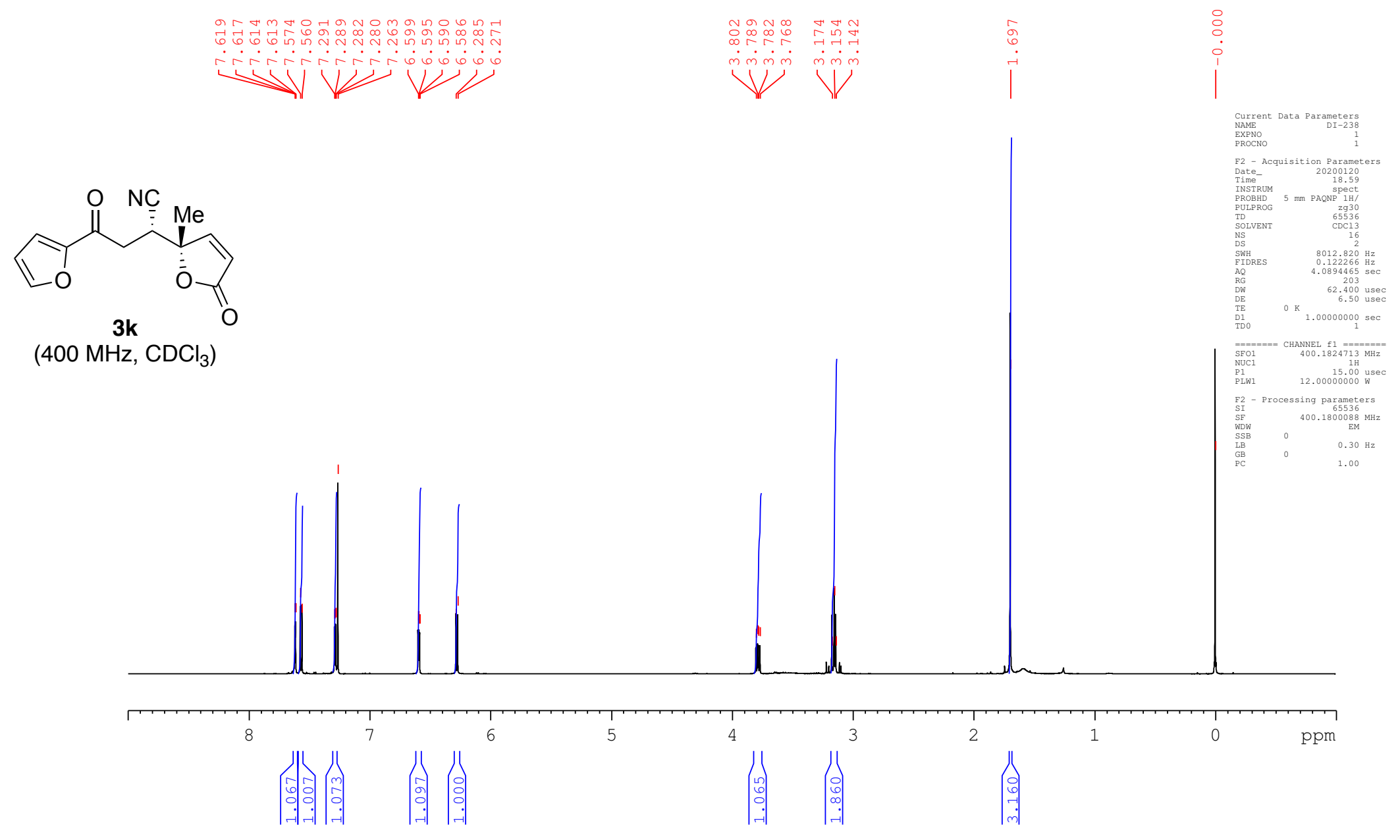




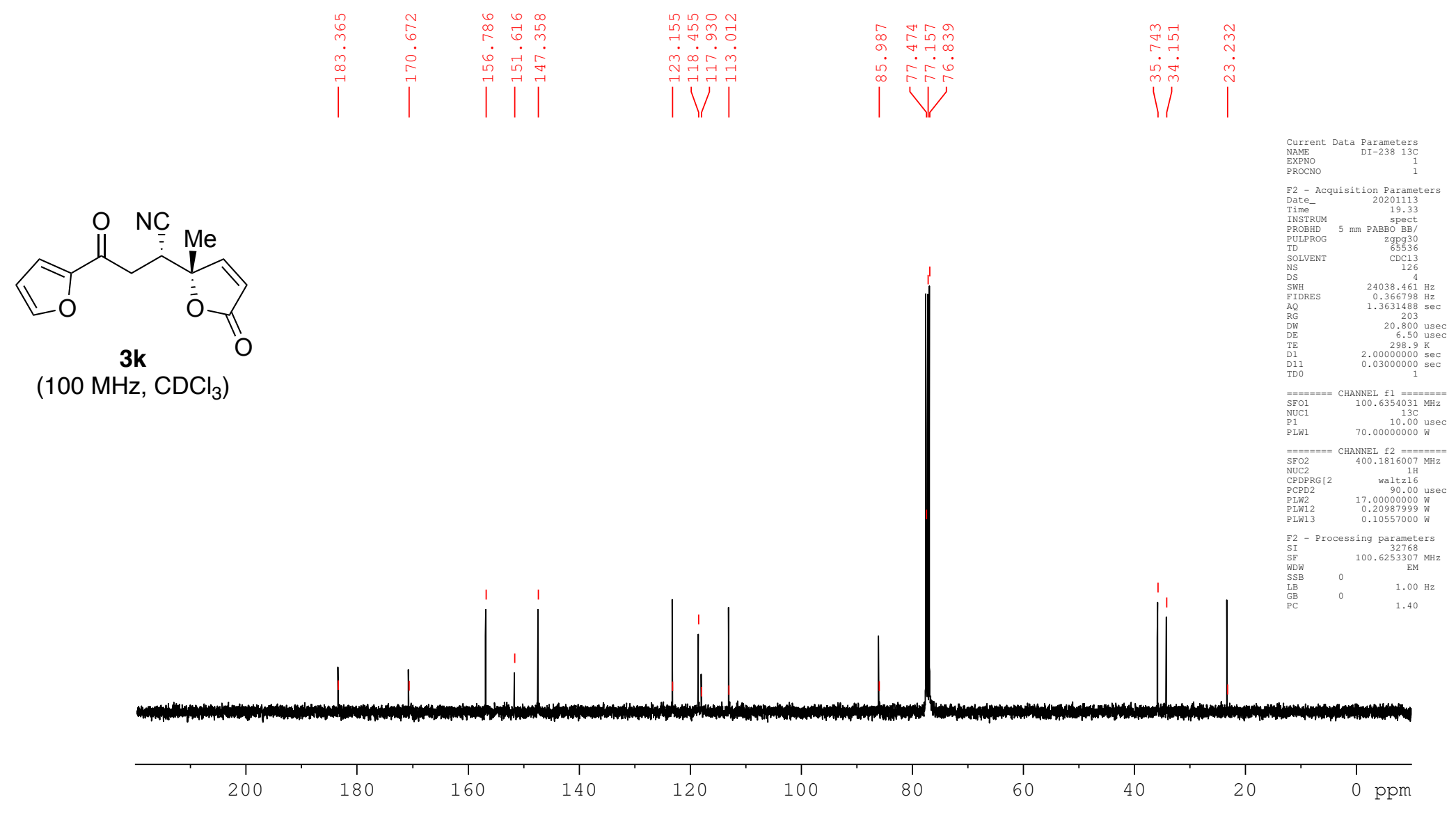




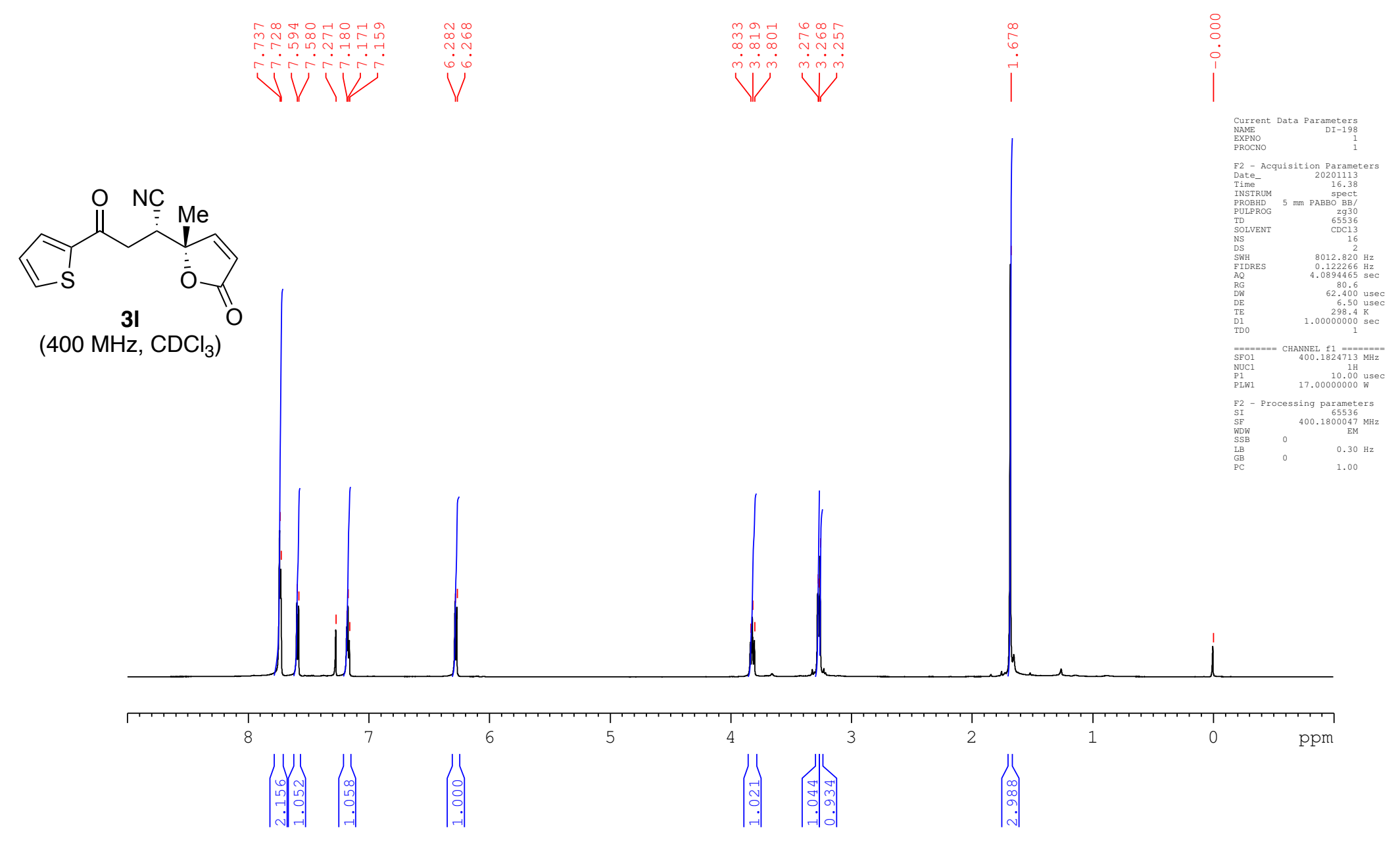




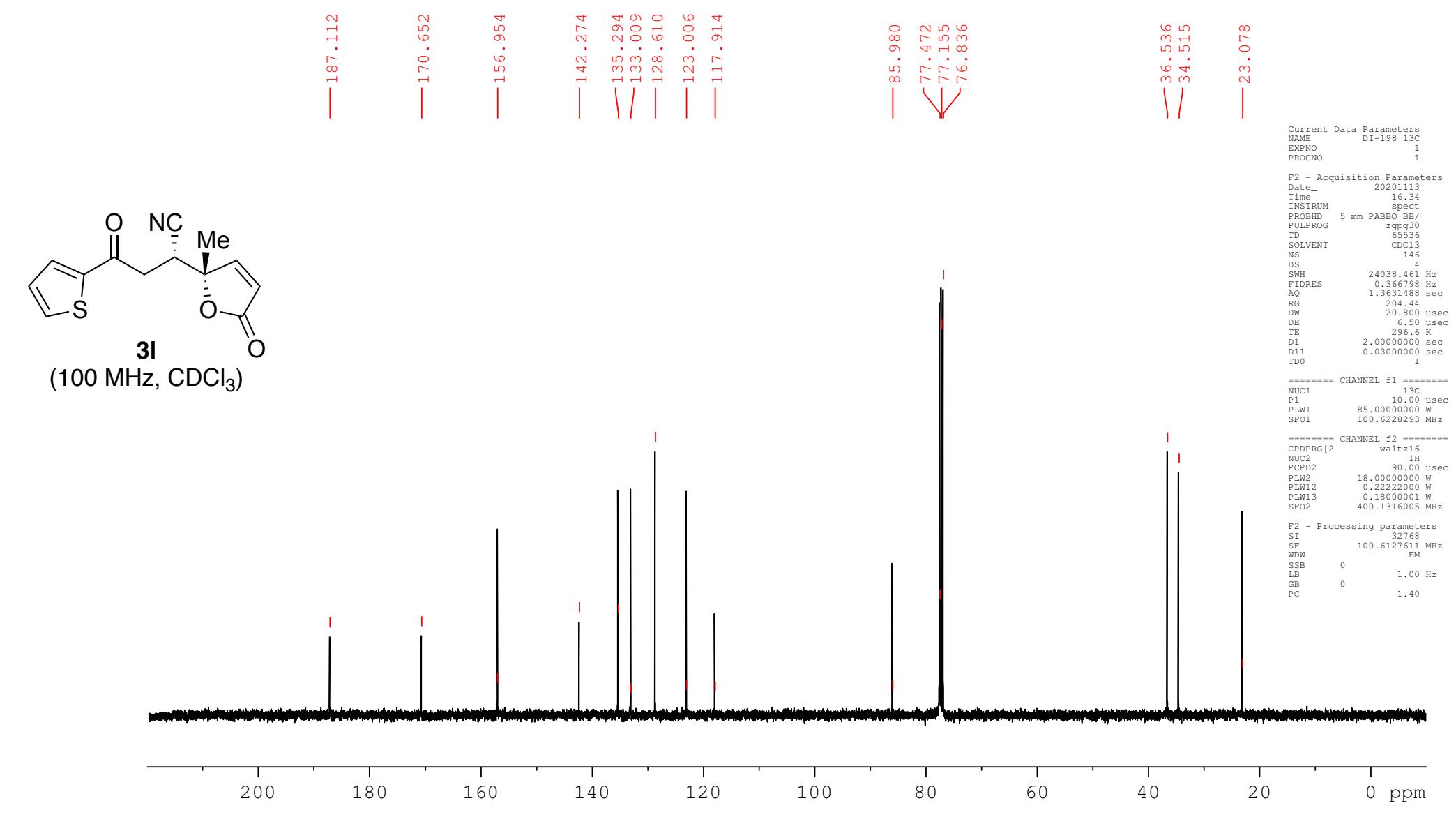




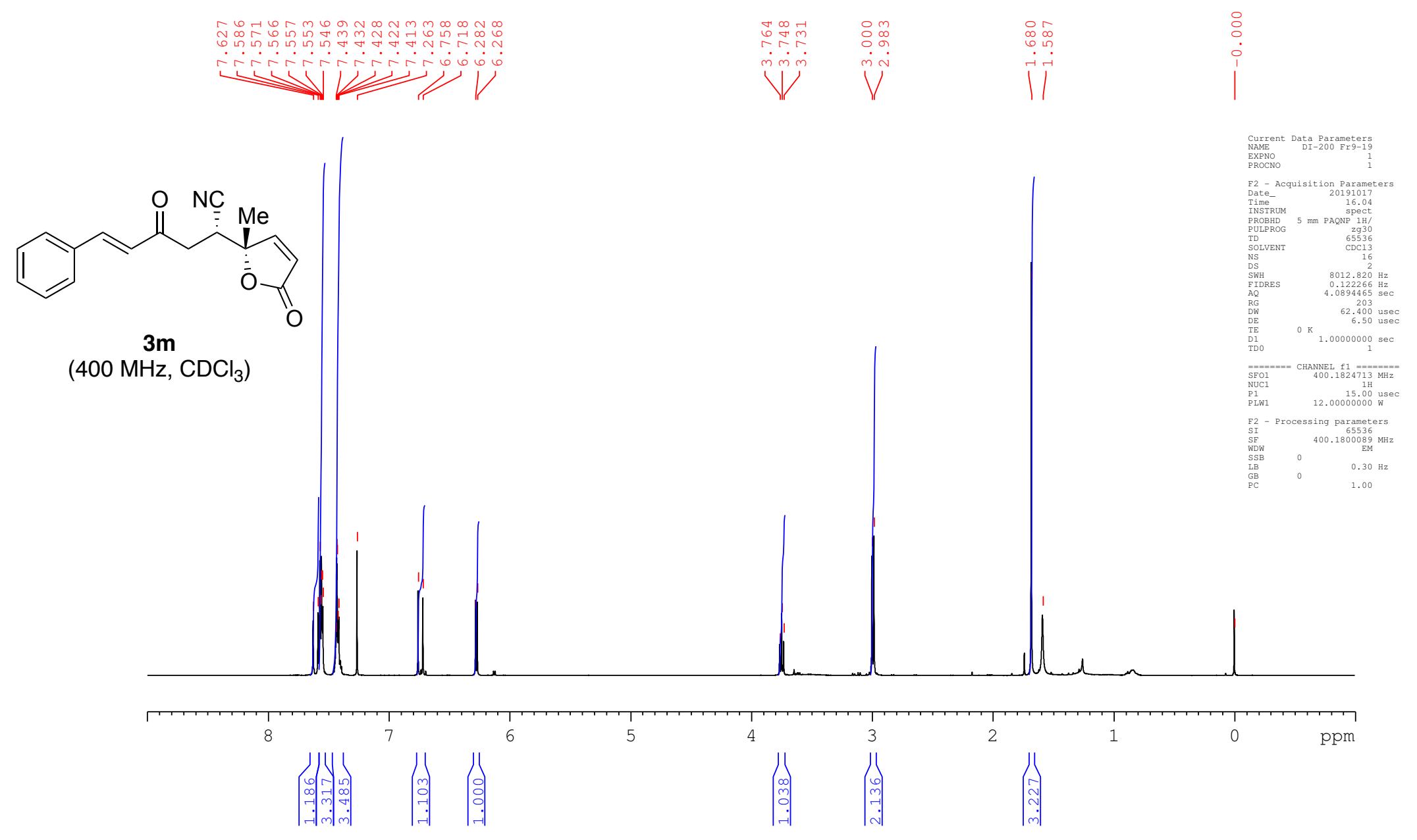




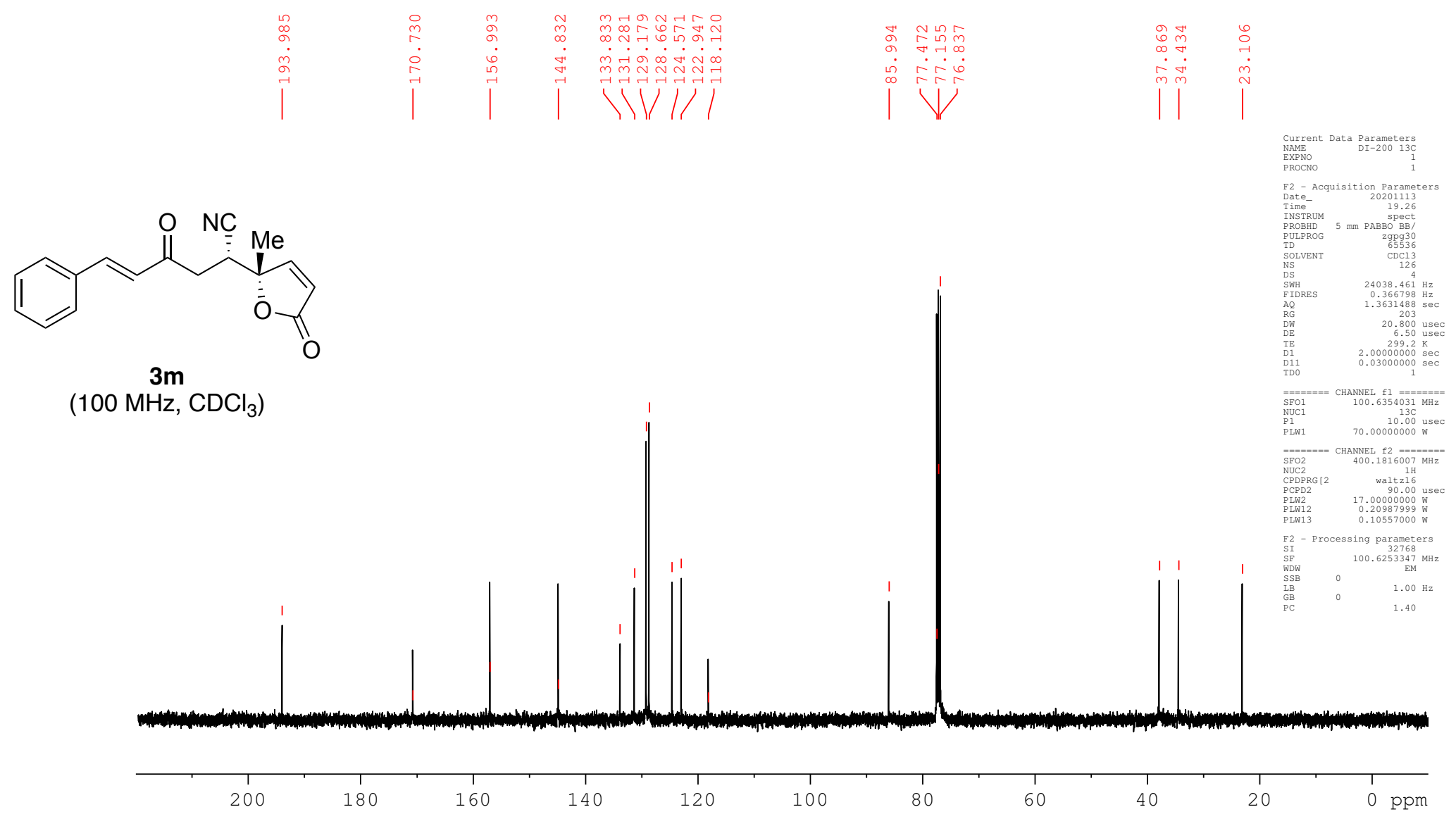




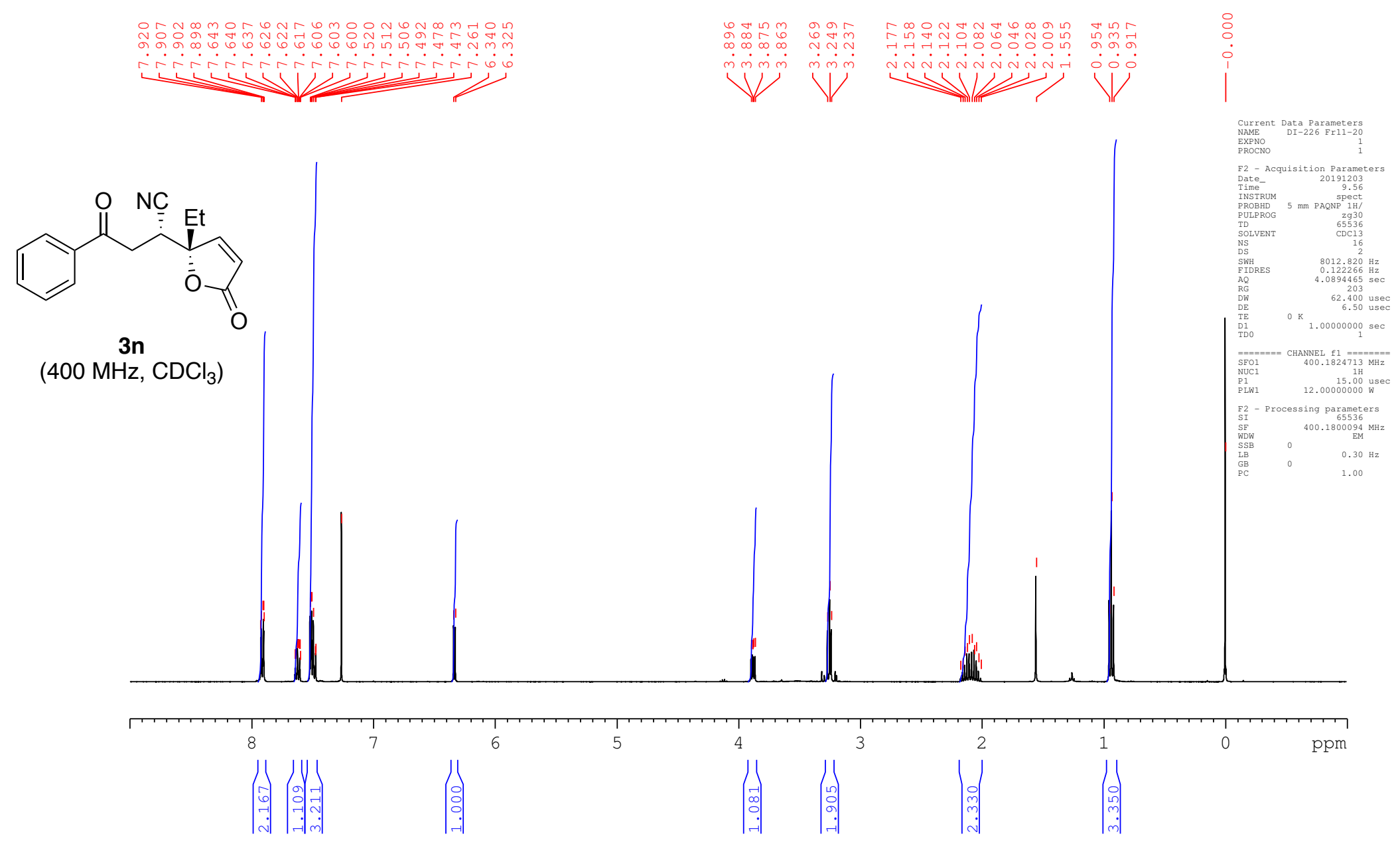




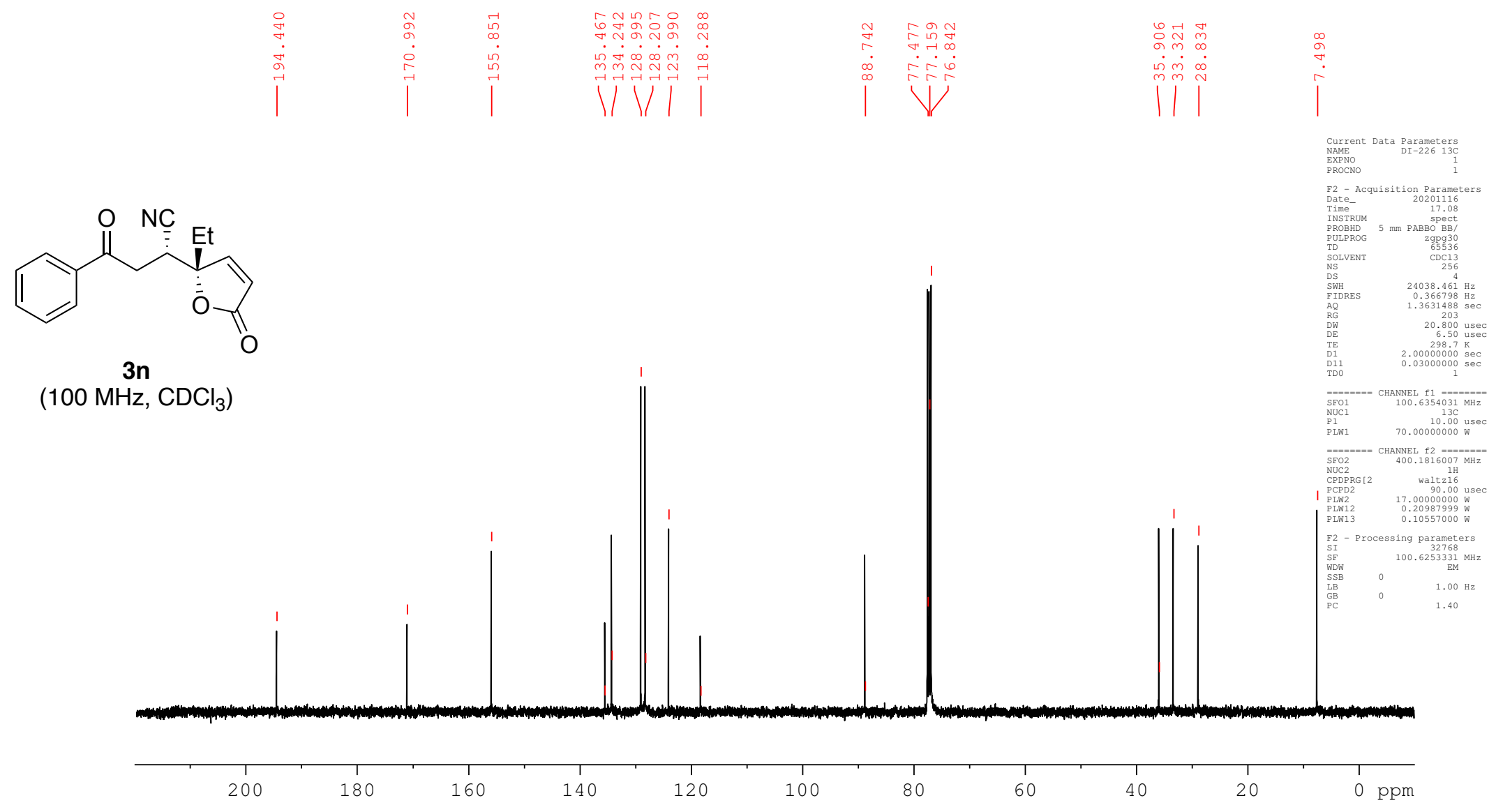




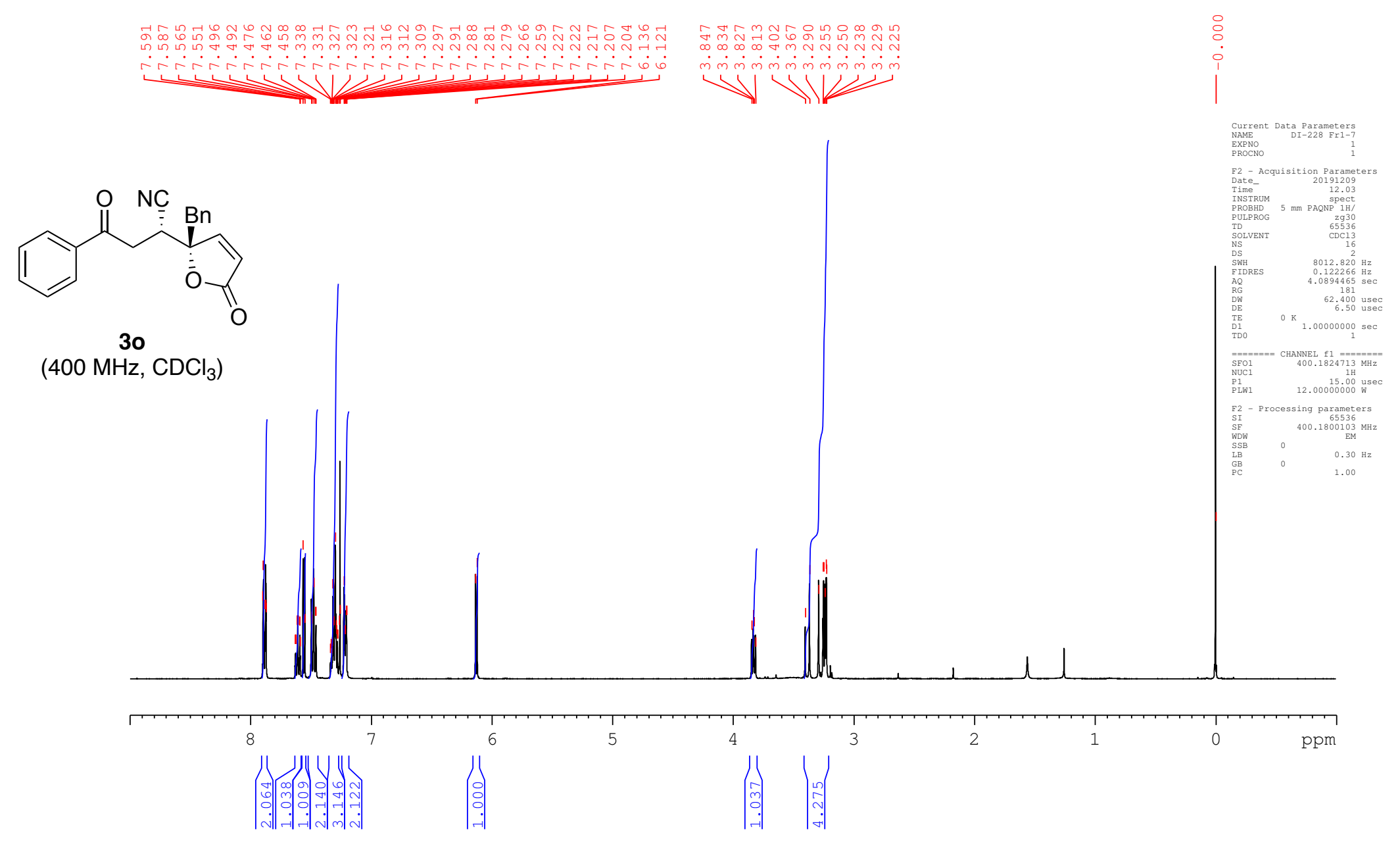




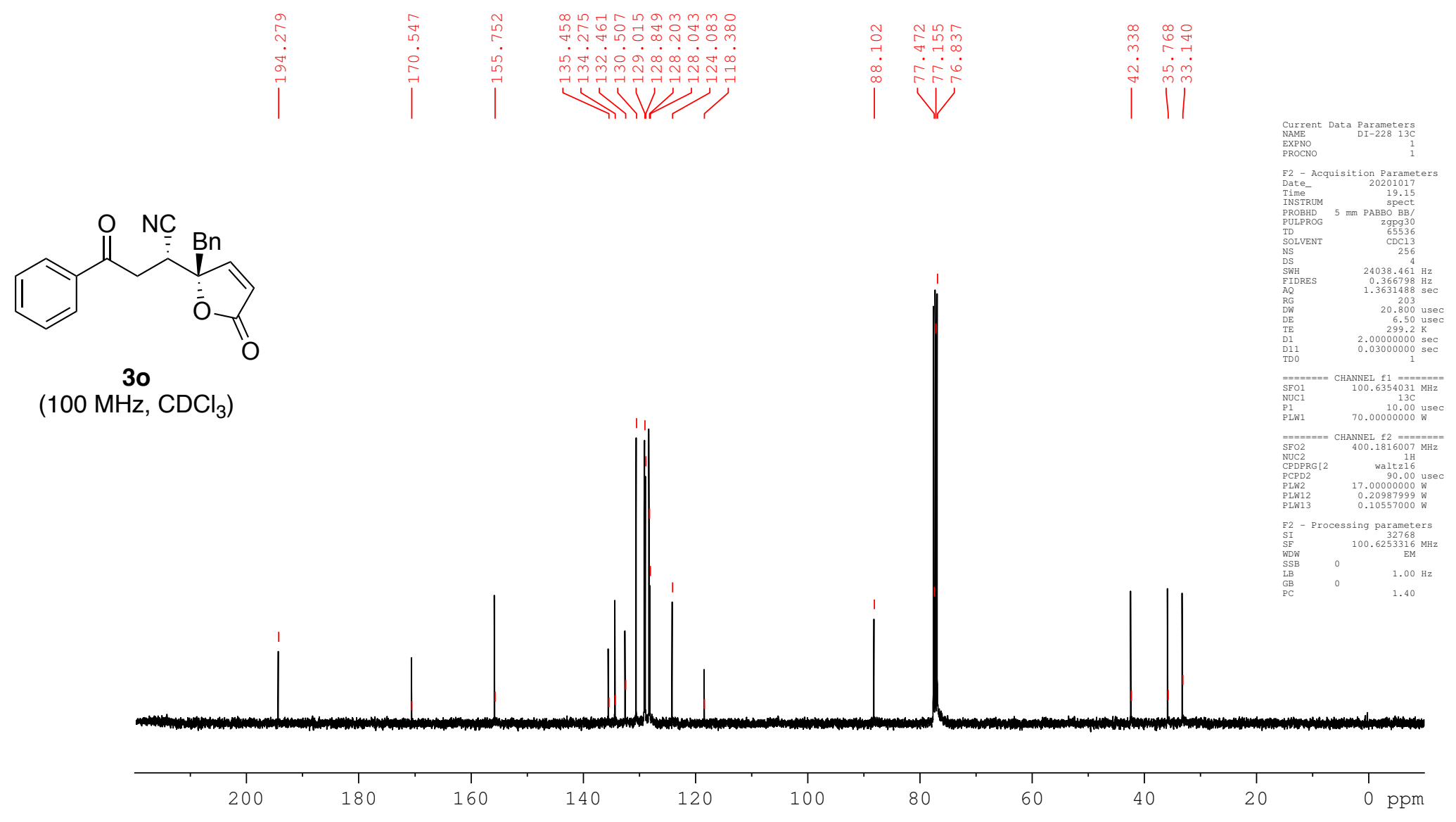




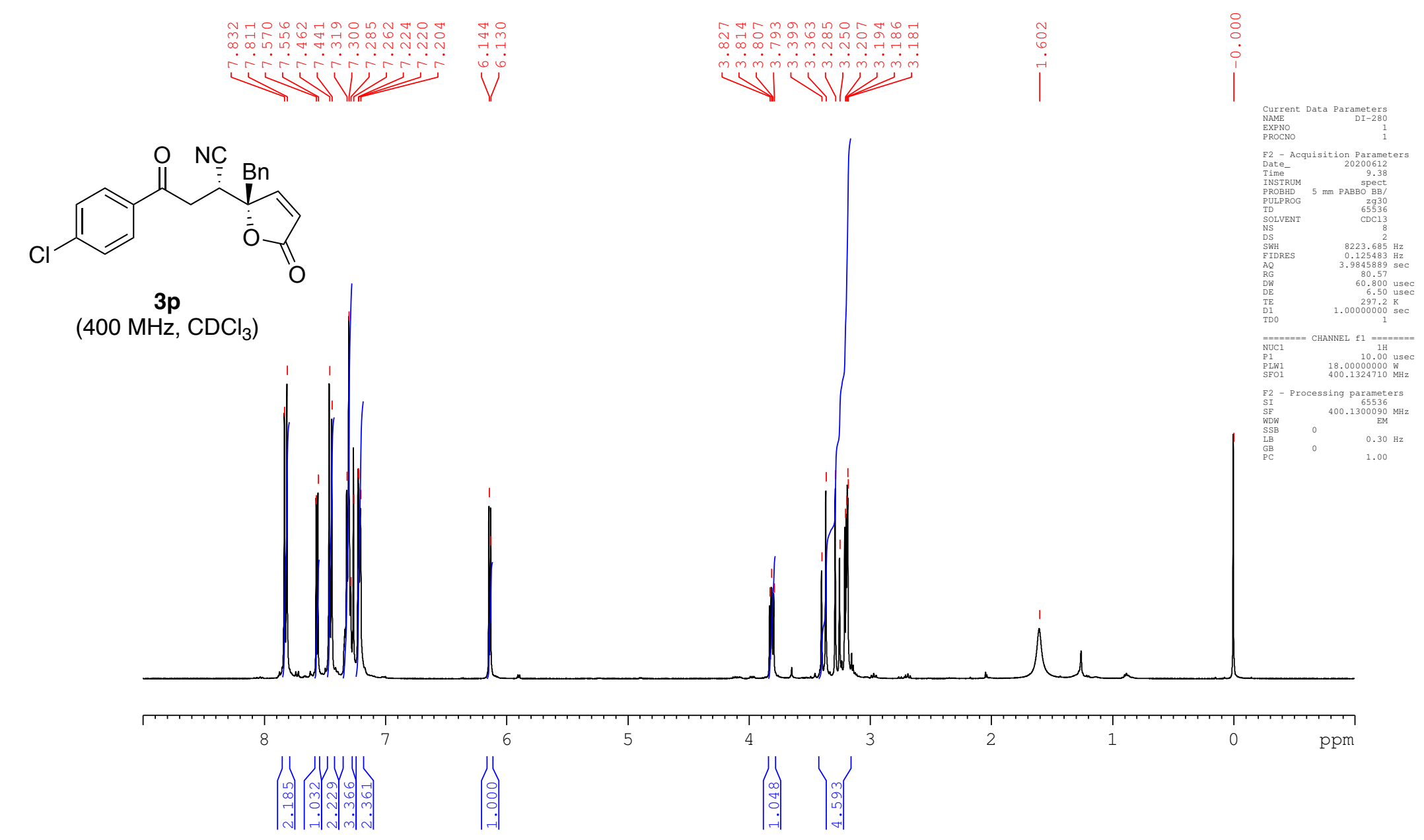




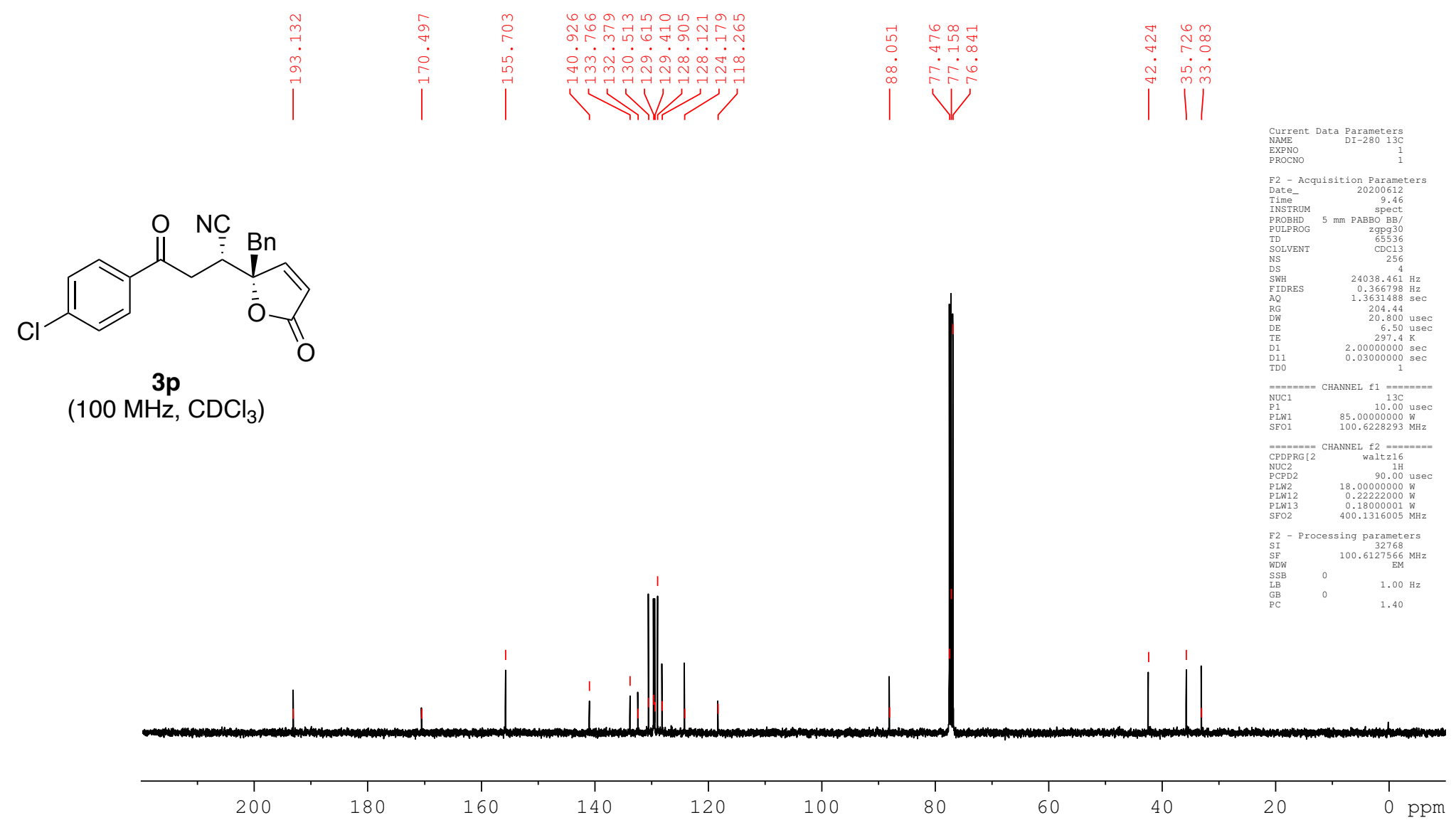




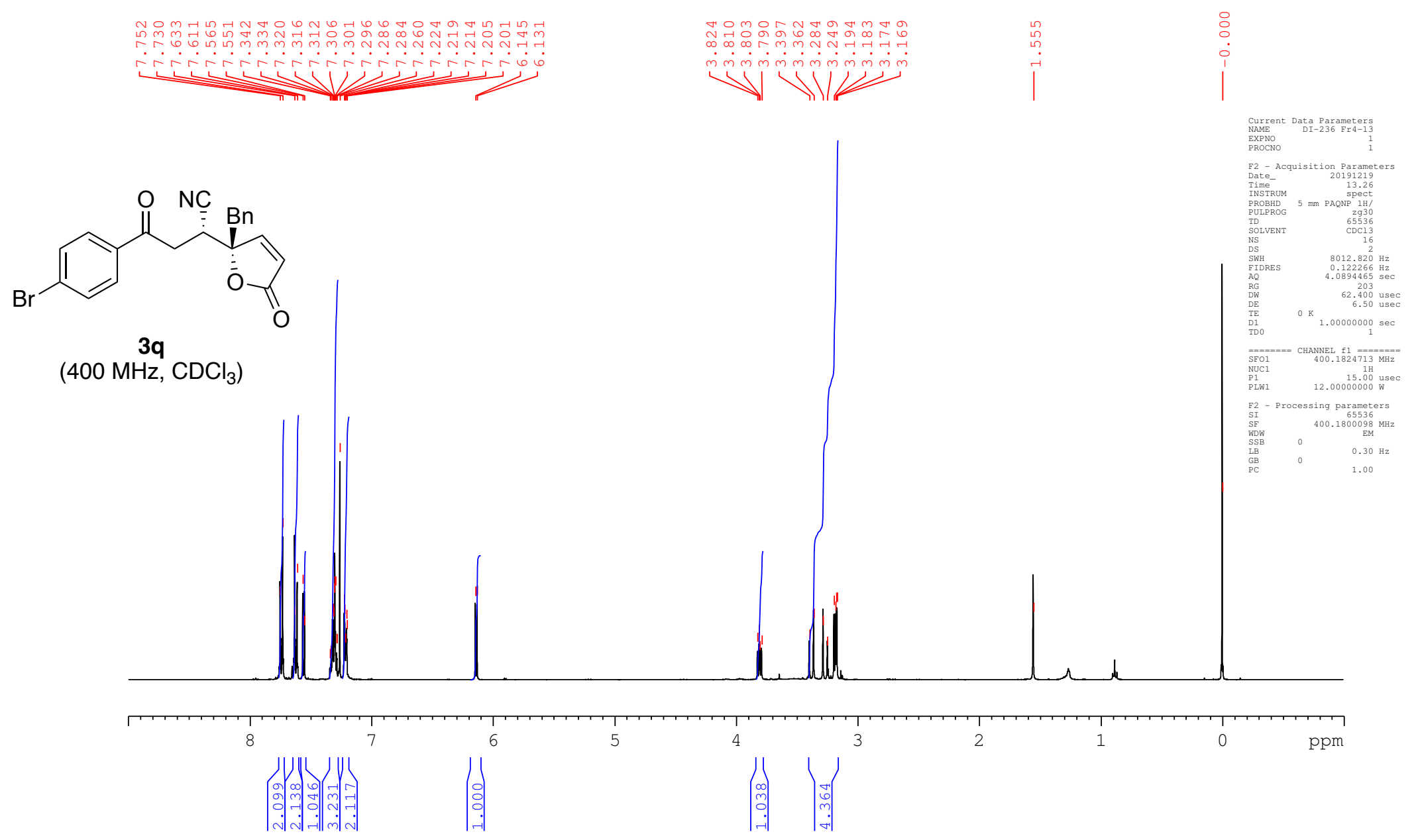




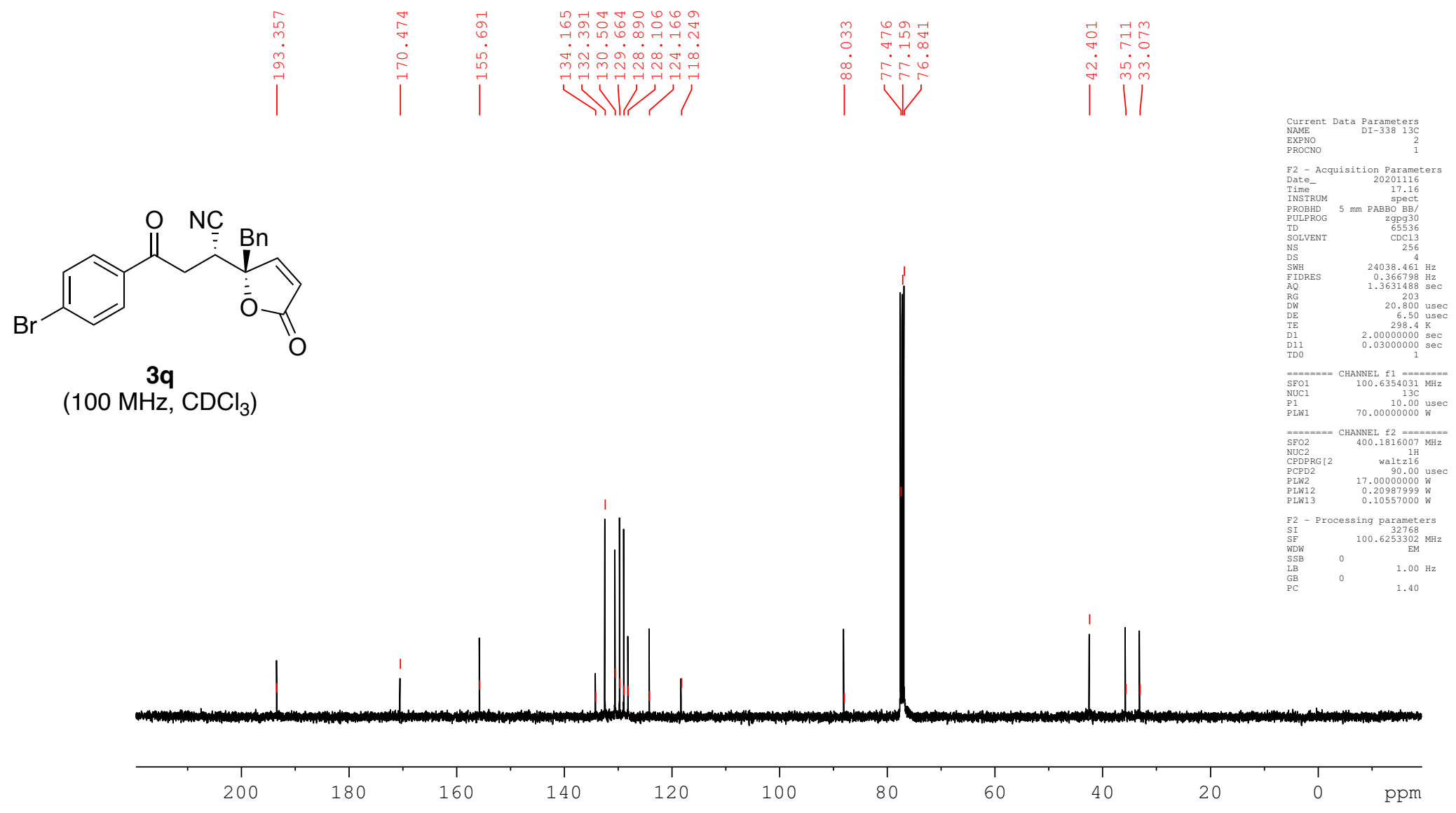




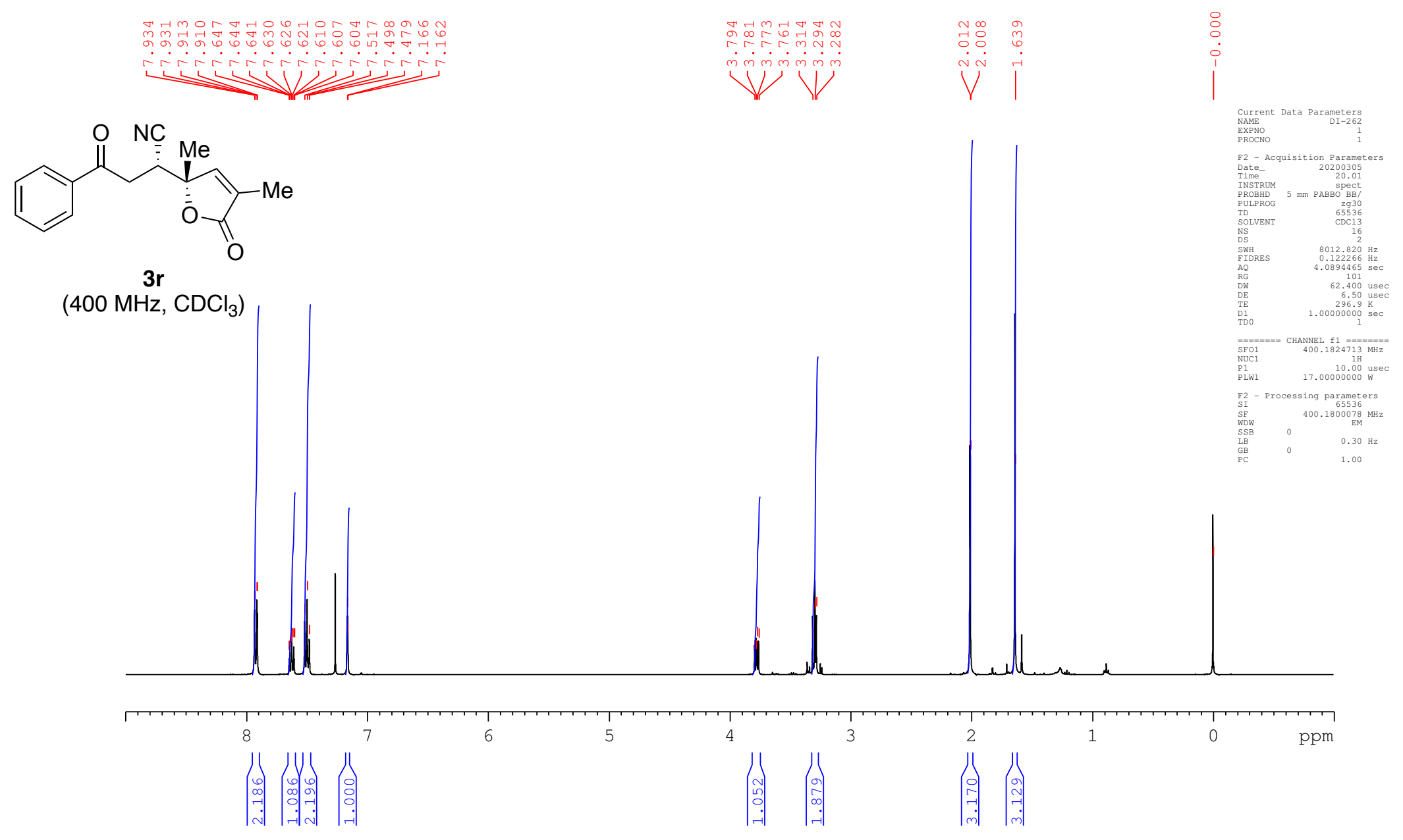




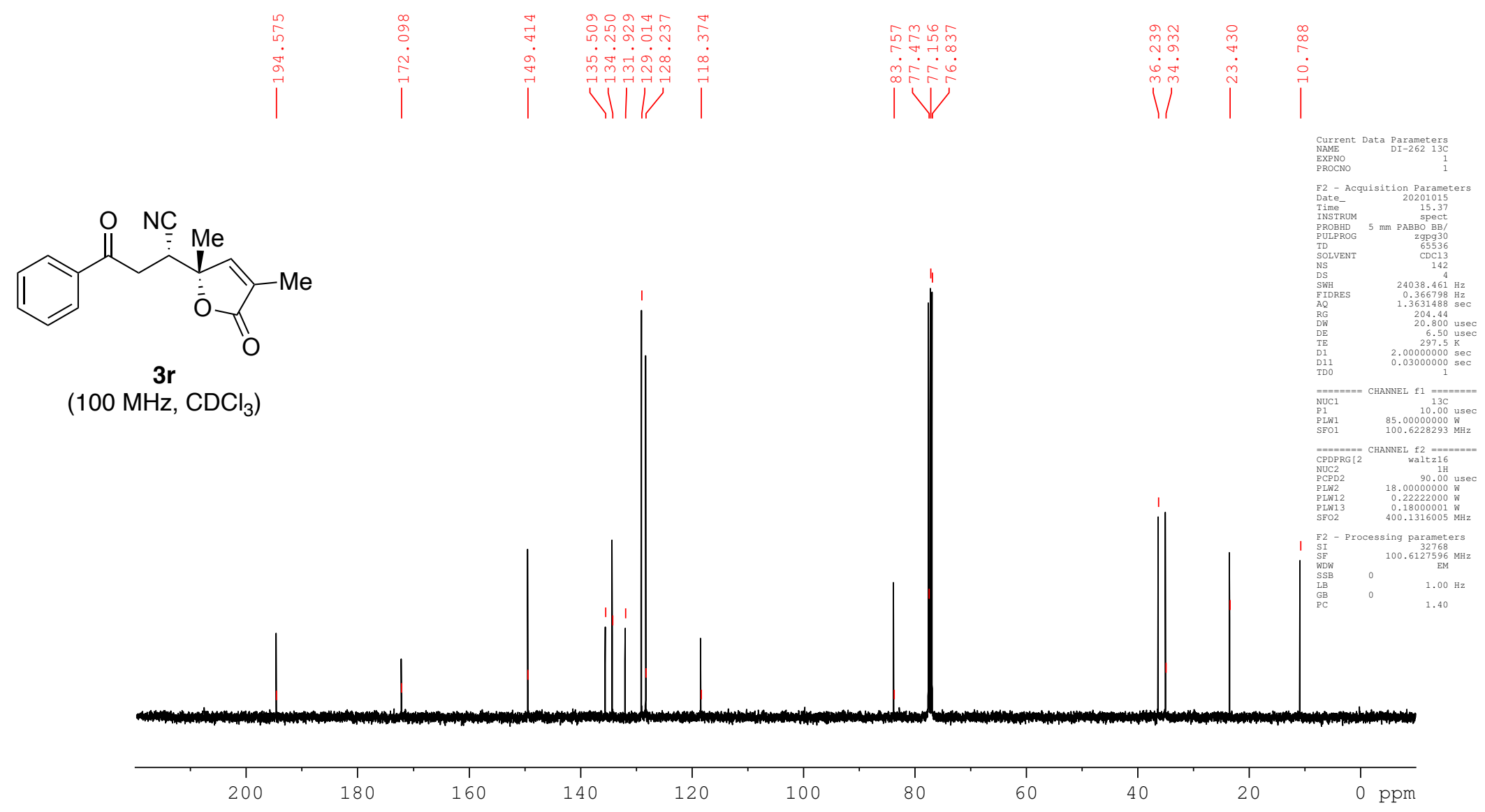




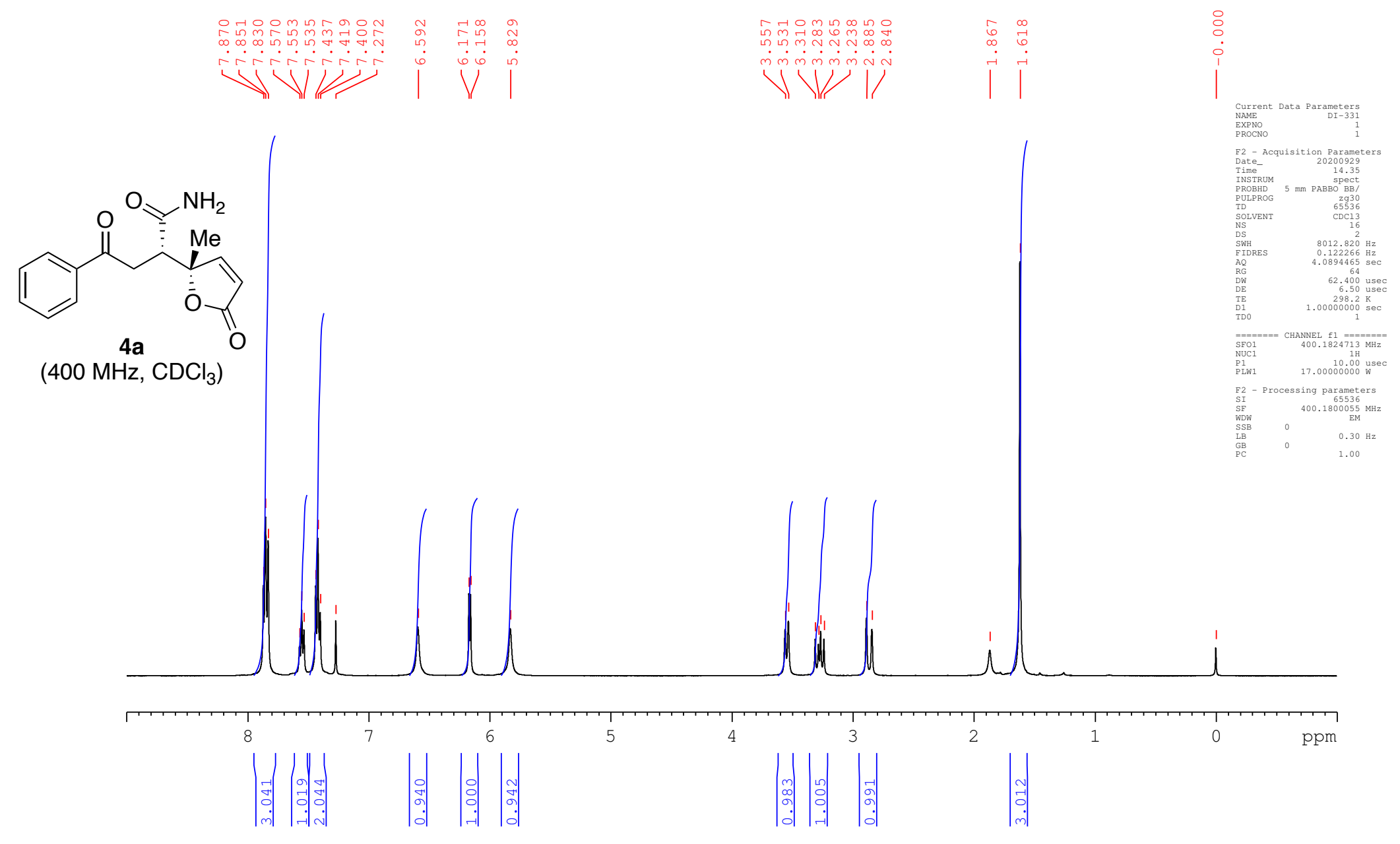




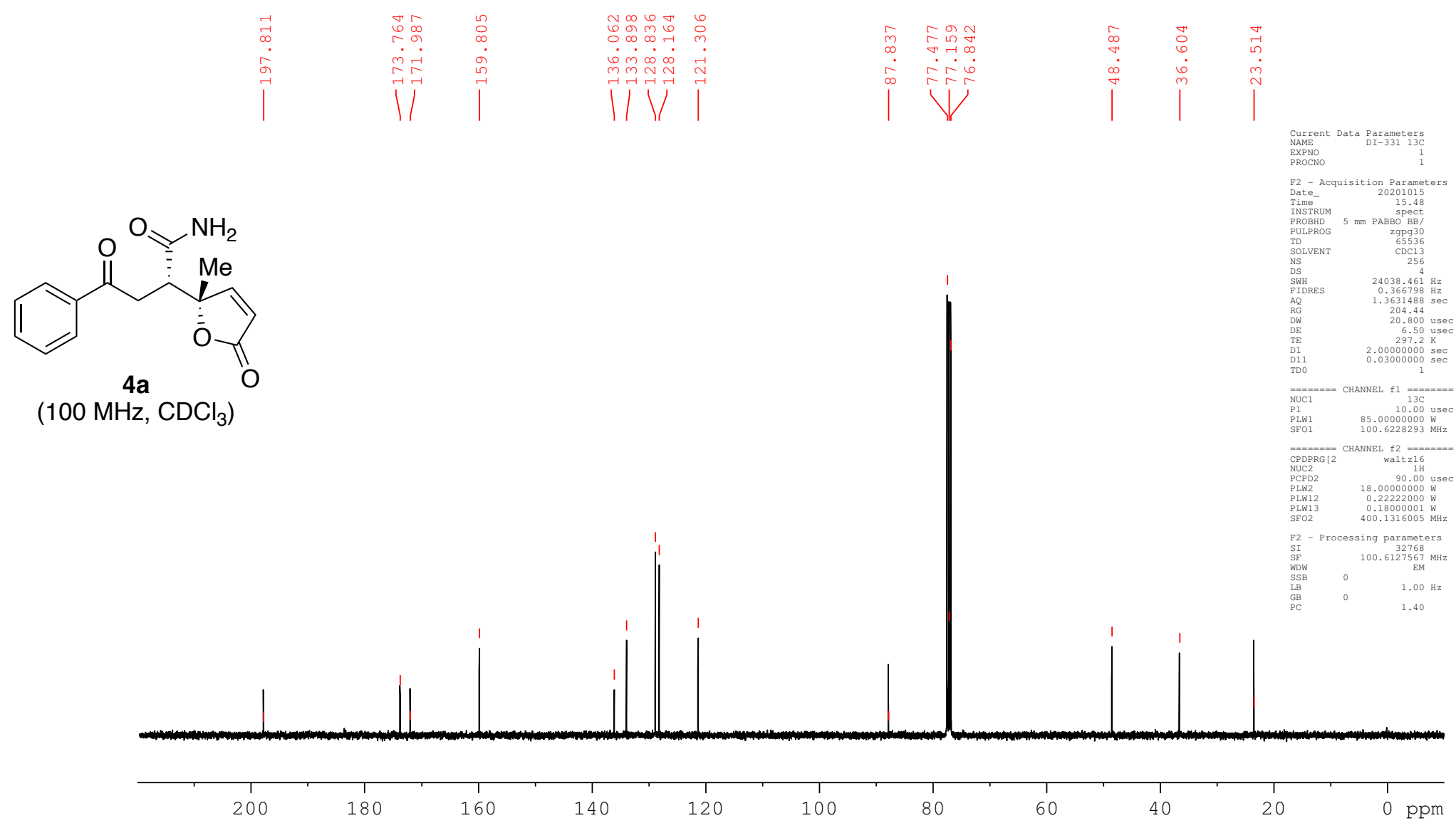




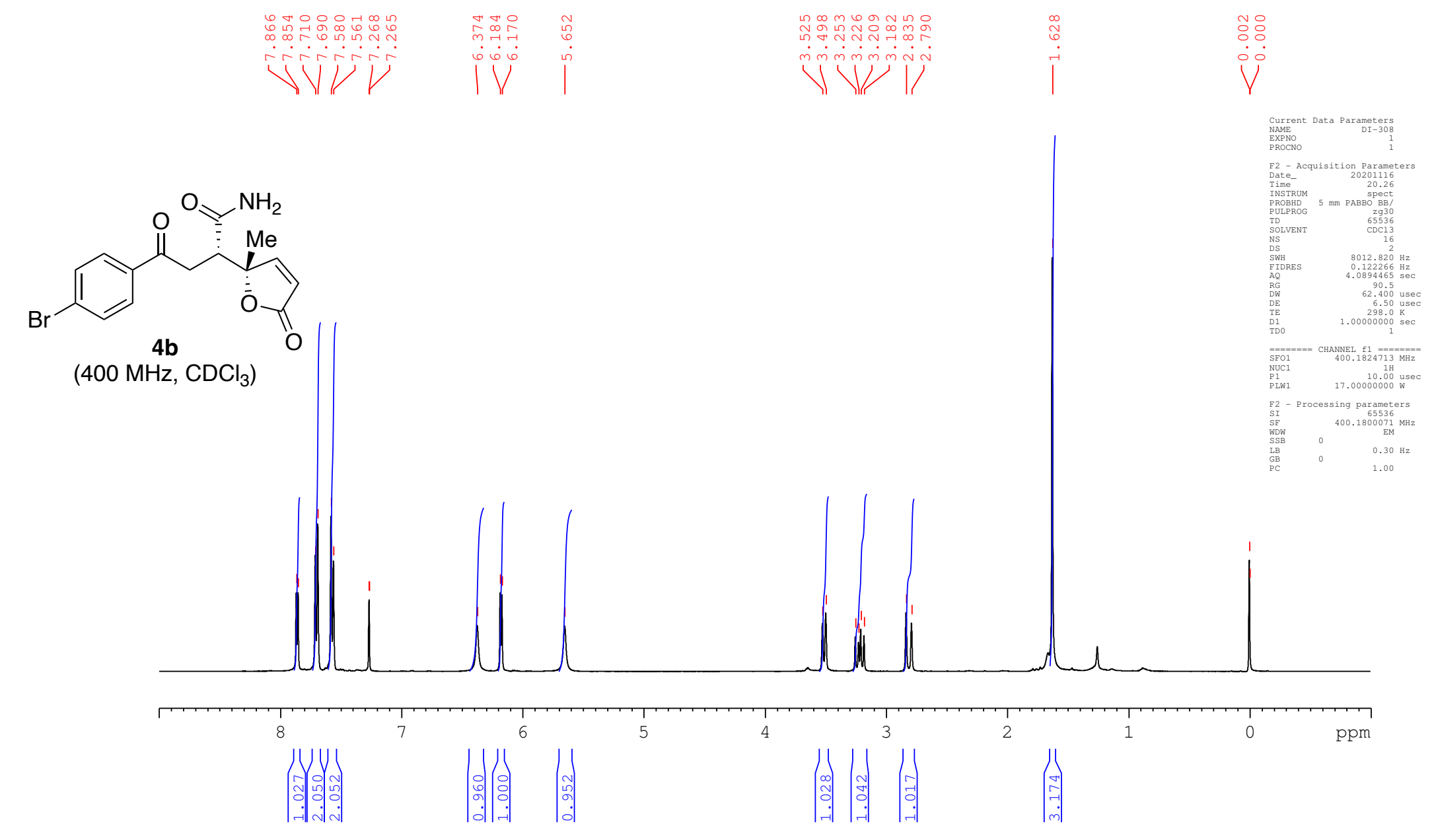




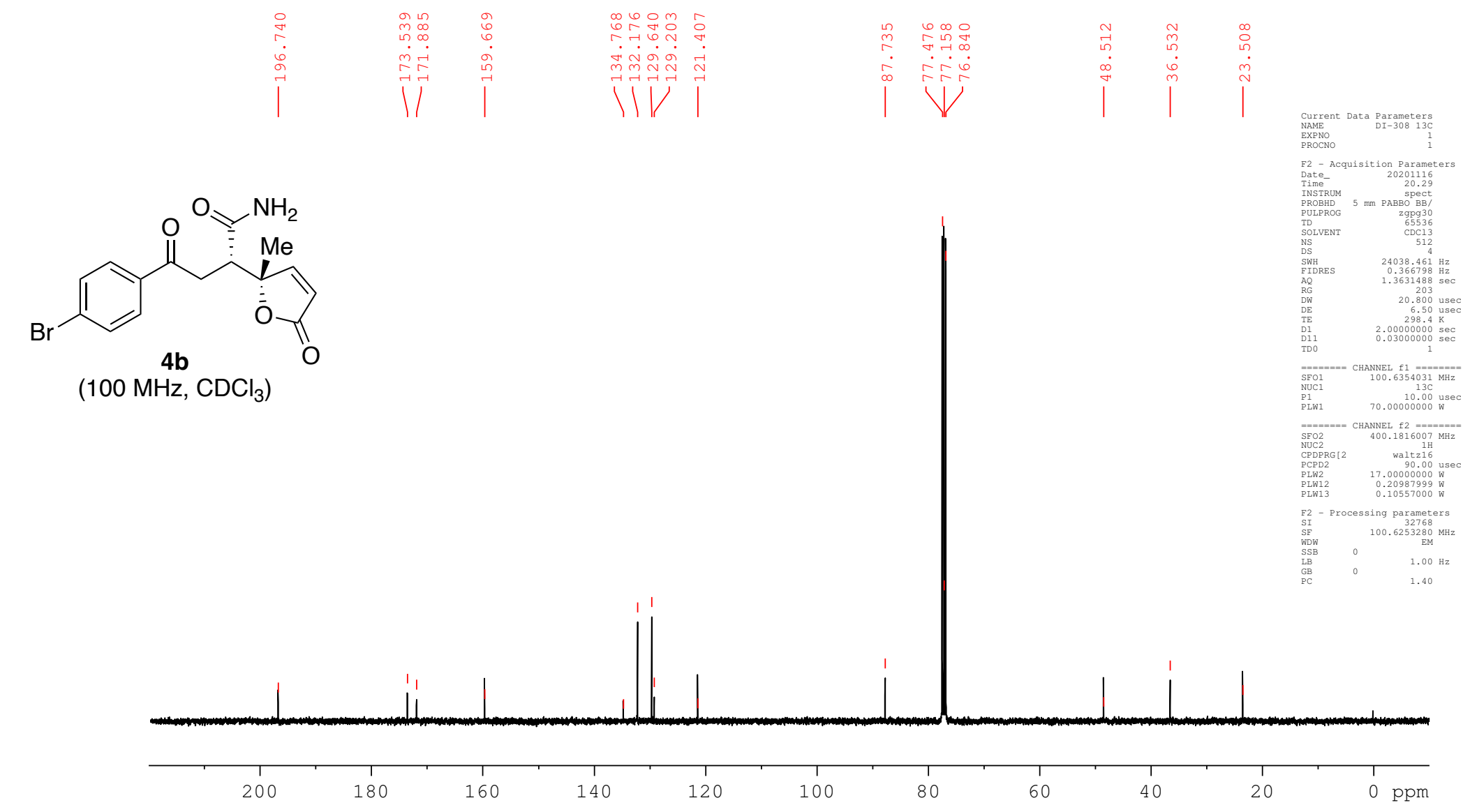




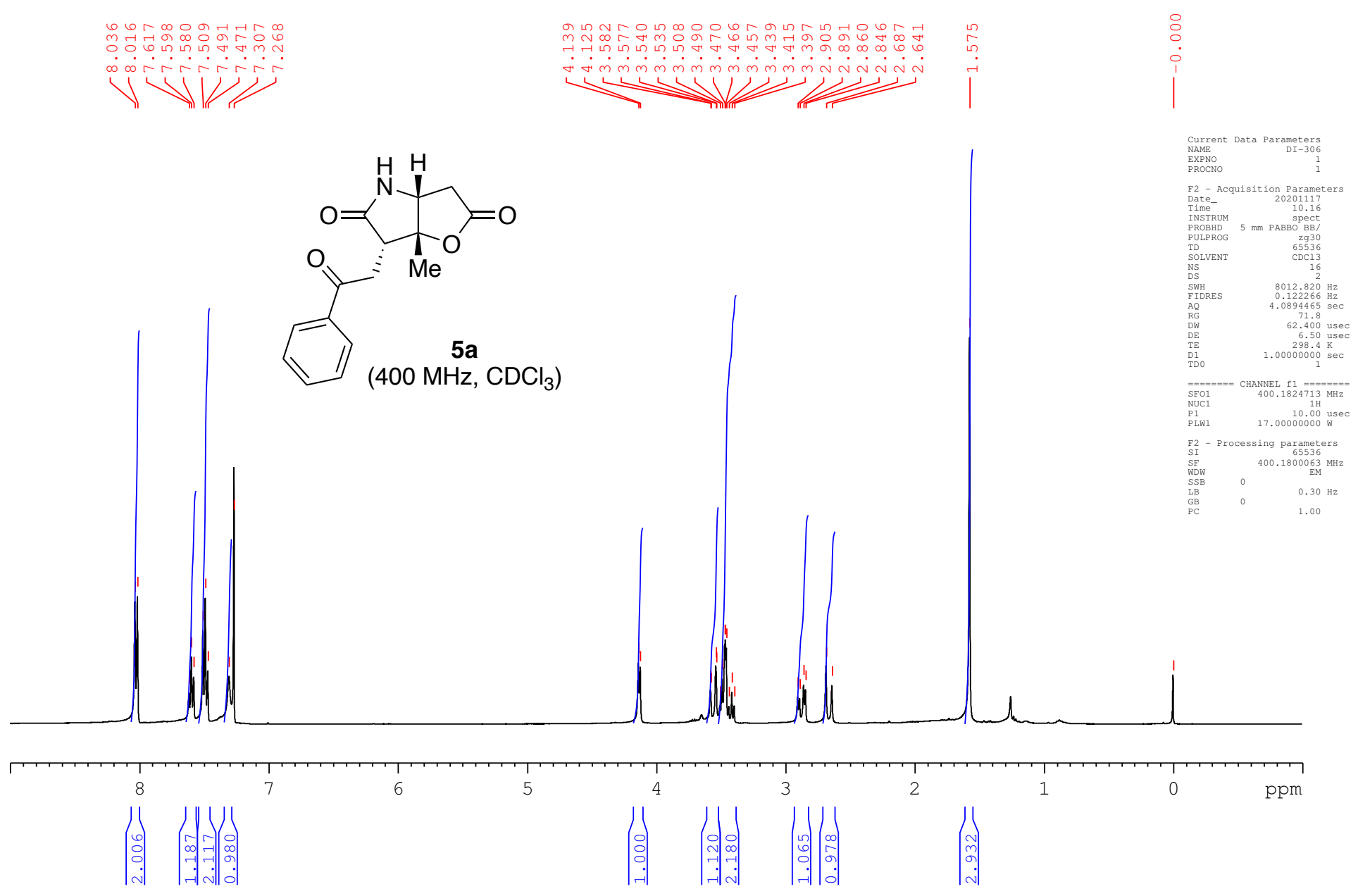




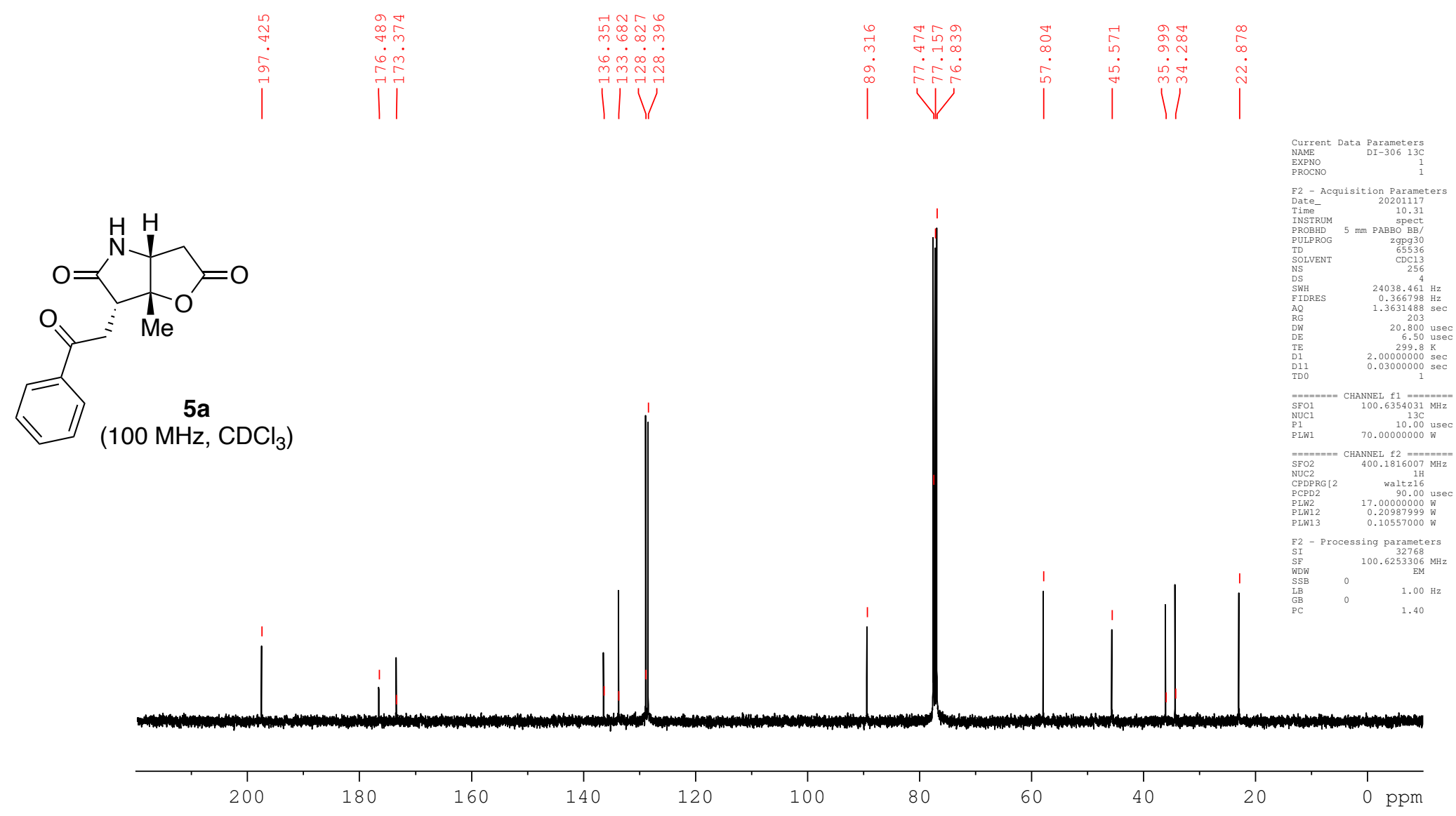




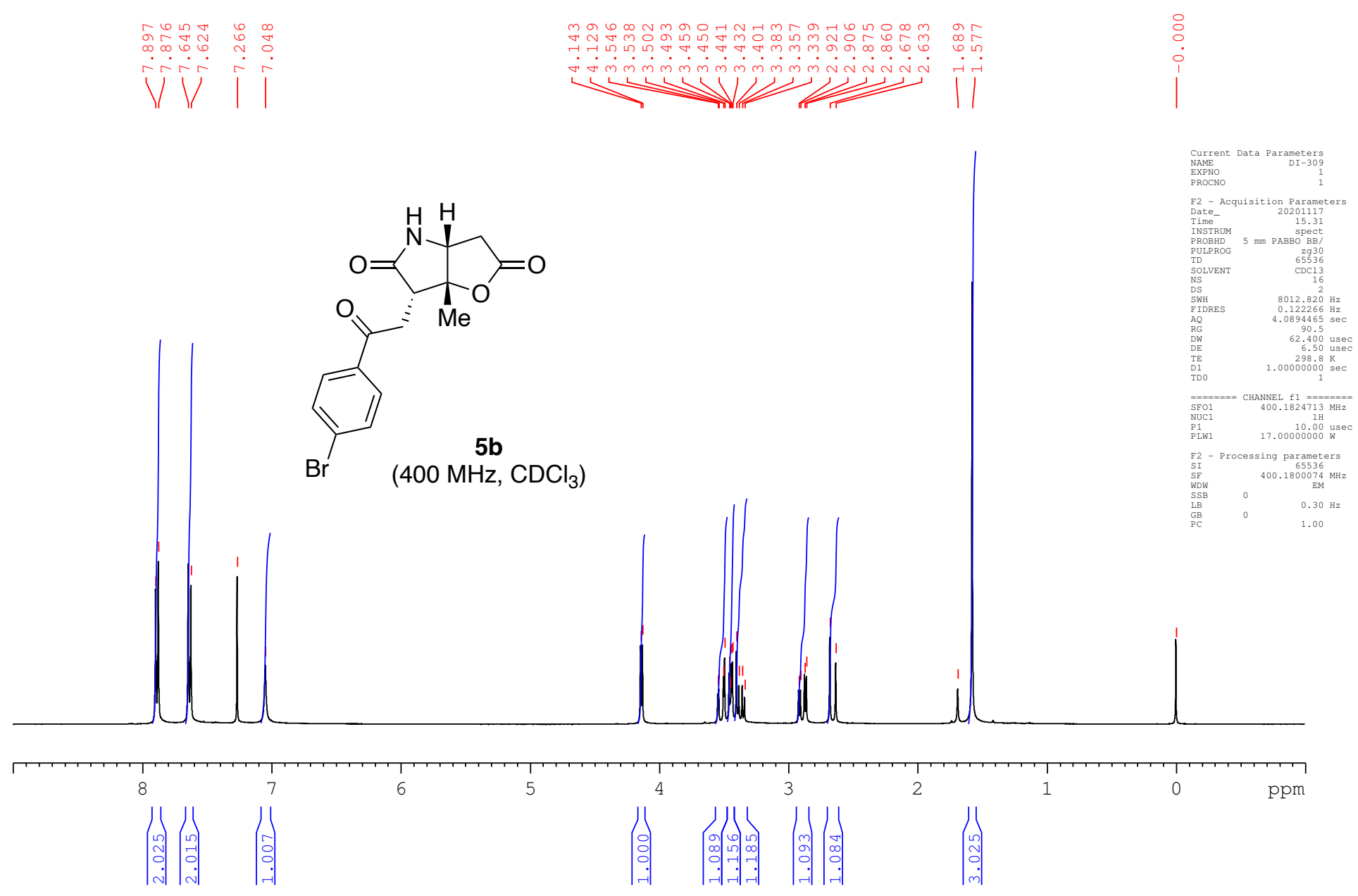




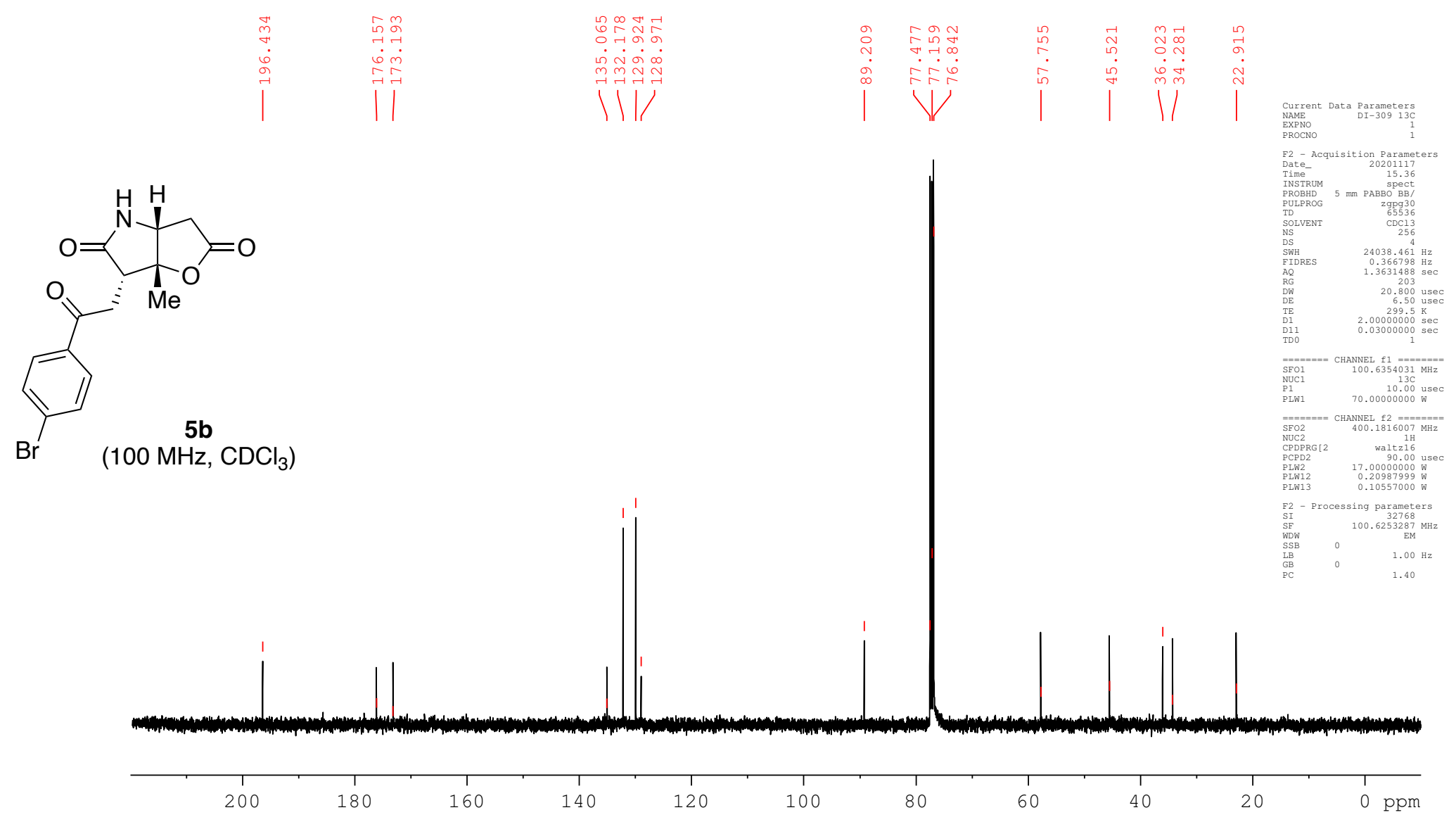




\section{HPLC Chromatograms}

The extra peaks of the inseparable impurities were observed in some racemic highperformance liquid chromatography (HPLC) charts. However, the by-products could not be removed by several columns chromatography. The HPLC charts were recorded using a photodiode array detector (PDA). Each racemic peak was also identified by the corresponding ultraviolet spectrum using the PDA.

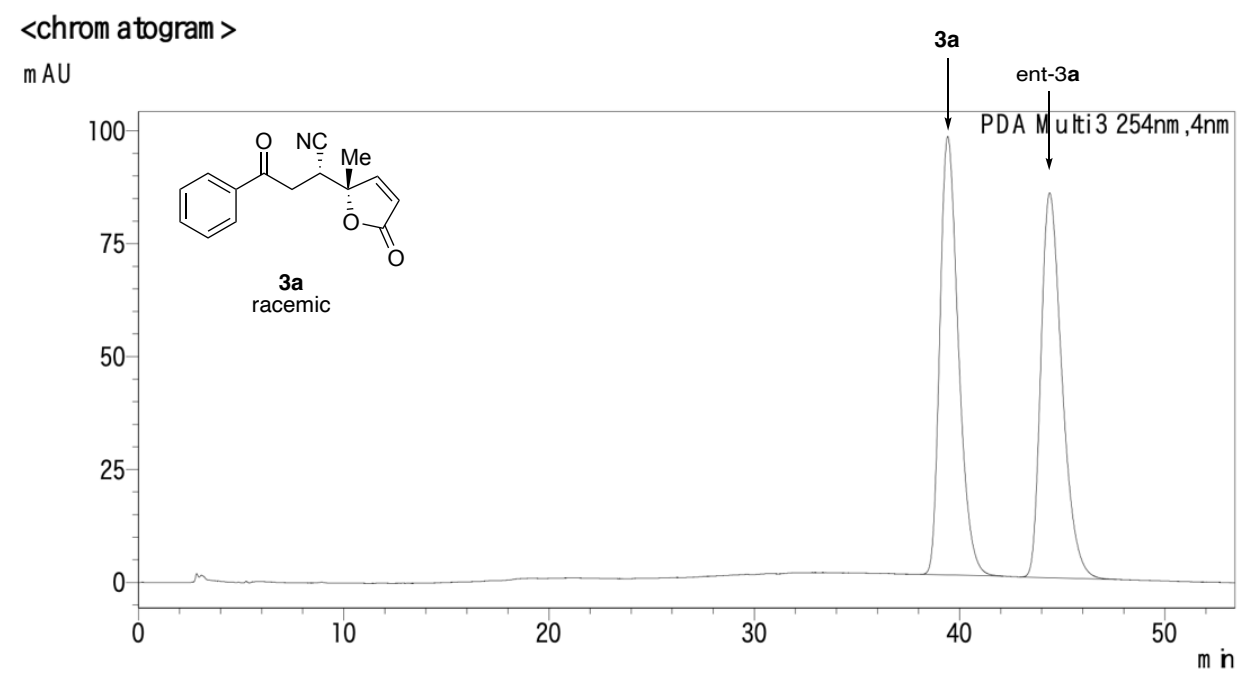

$<$ peak report

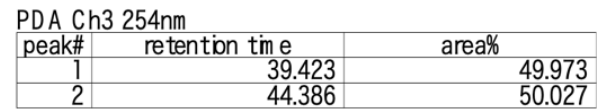

$<$ chrom atogram >

$\mathrm{mAU}$

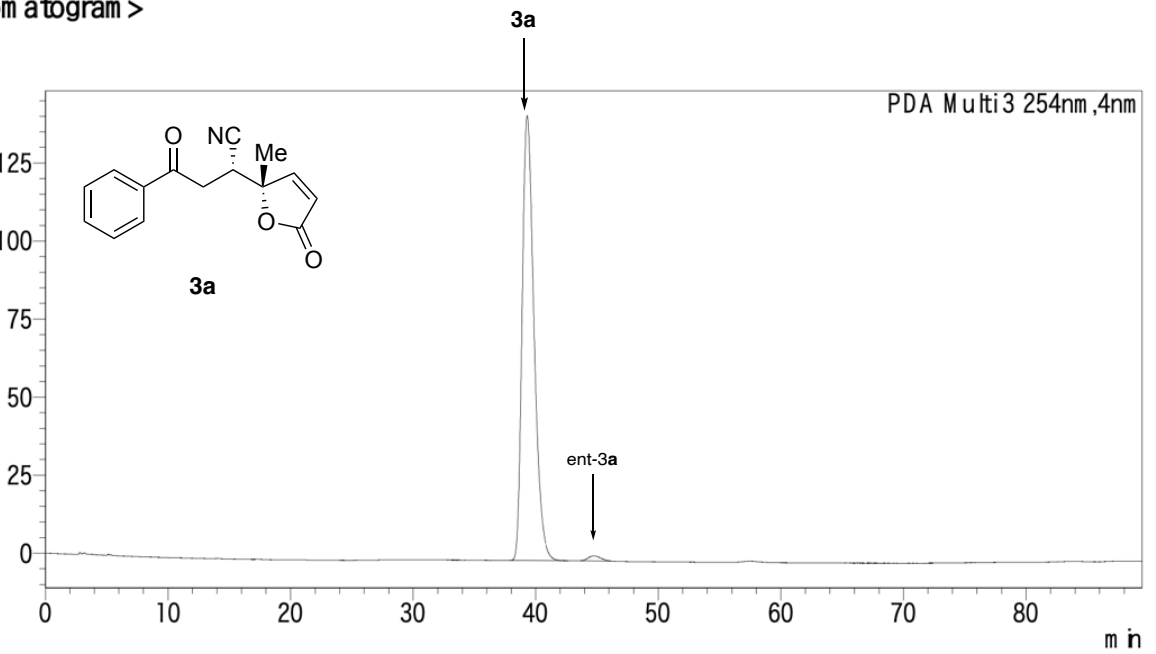

$<$ peak report

PDA Ch3 254nm

\begin{tabular}{|r|r|r|}
\hline peak\# & retention tim e & area\% \\
\hline 1 & 39.292 & 98.815 \\
\hline 2 & 44.728 & 1.185 \\
\hline
\end{tabular} 
$<$ chrom atogram >

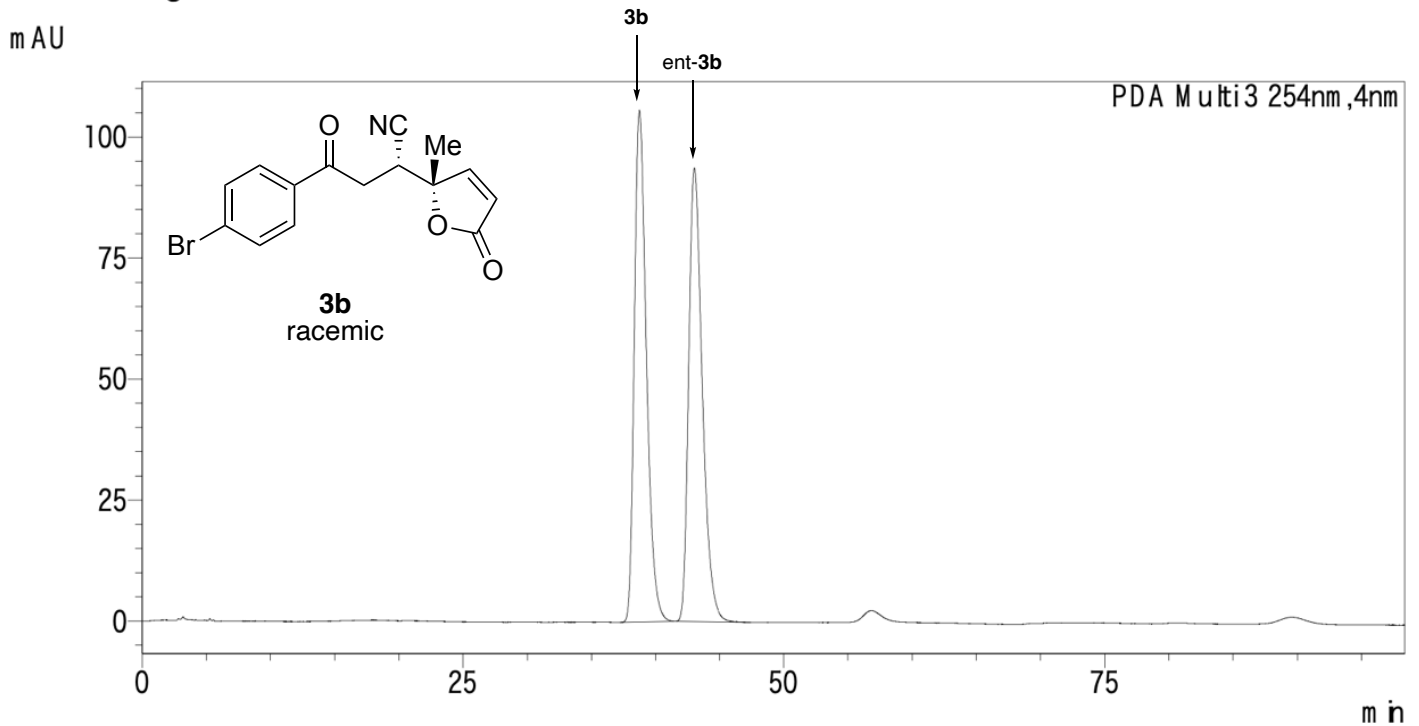

$<$ peak report

PDA Ch3 254nm

\begin{tabular}{|r|r|r|}
\hline peak\# & retention tim e & area\% \\
\hline 1 & 38.736 & 50.031 \\
\hline 2 & 43.016 & 49.969 \\
\hline
\end{tabular}

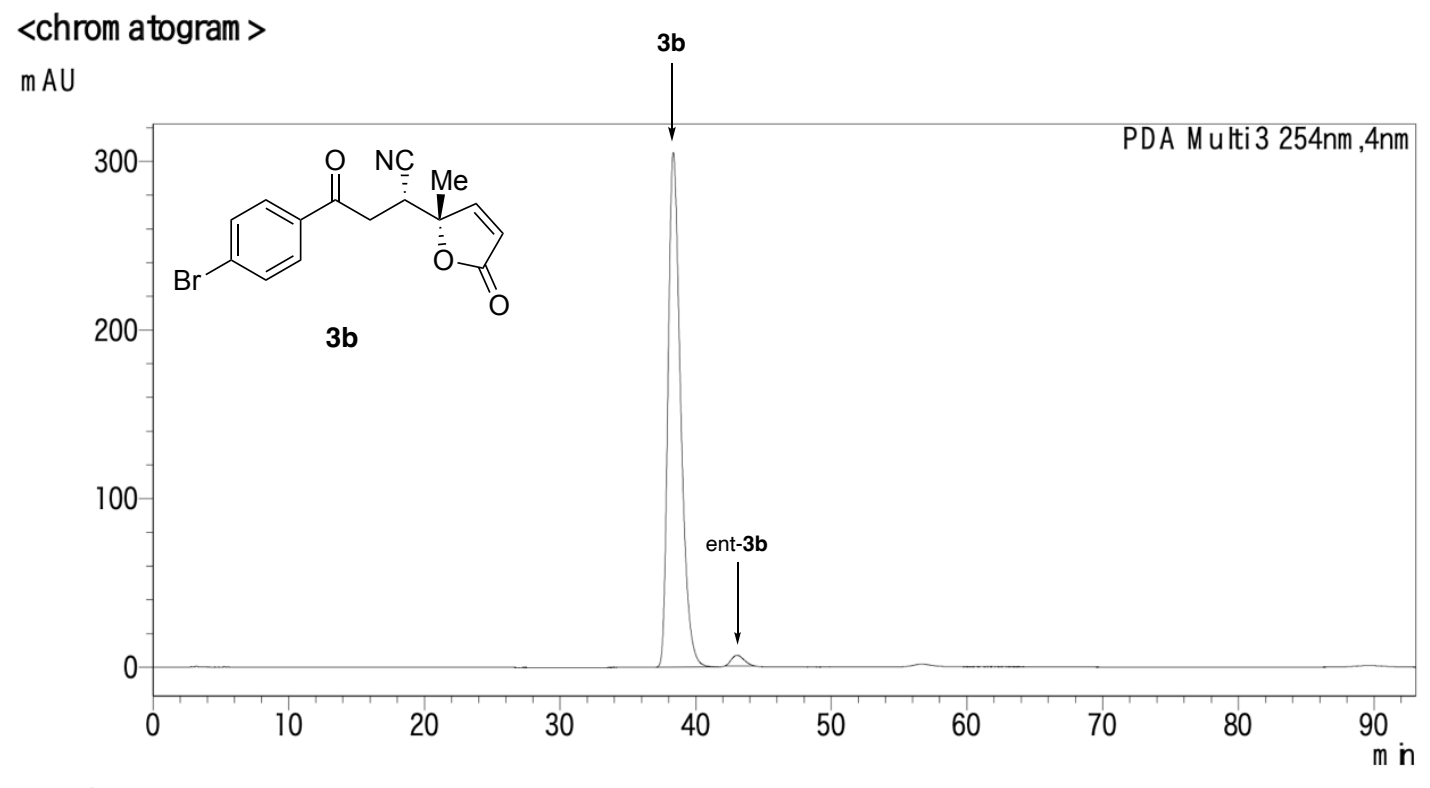

$<$ peak report

PDA Ch3 254nm

\begin{tabular}{|r|r|r|}
\hline peak\# & retention tim e & area\% \\
\hline 1 & 38.340 & 97.924 \\
\hline 2 & 43.051 & 2.076 \\
\hline
\end{tabular}




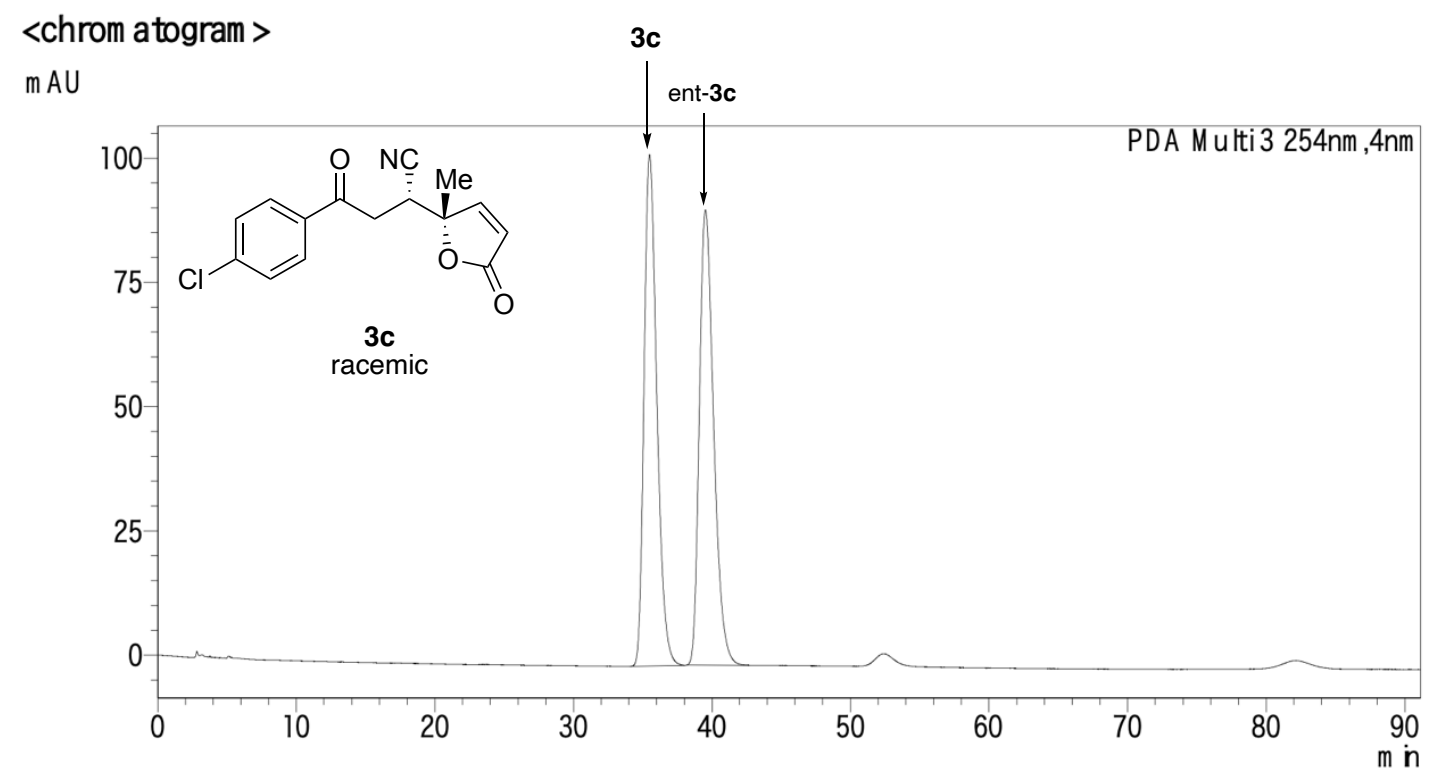

<peak report

PDA Ch3 254nm

\begin{tabular}{|r|r|r|}
\hline peak\# & retention tim e & area\% \\
\hline 1 & 35.483 & 50.013 \\
\hline 2 & 39.517 & 49.987 \\
\hline
\end{tabular}

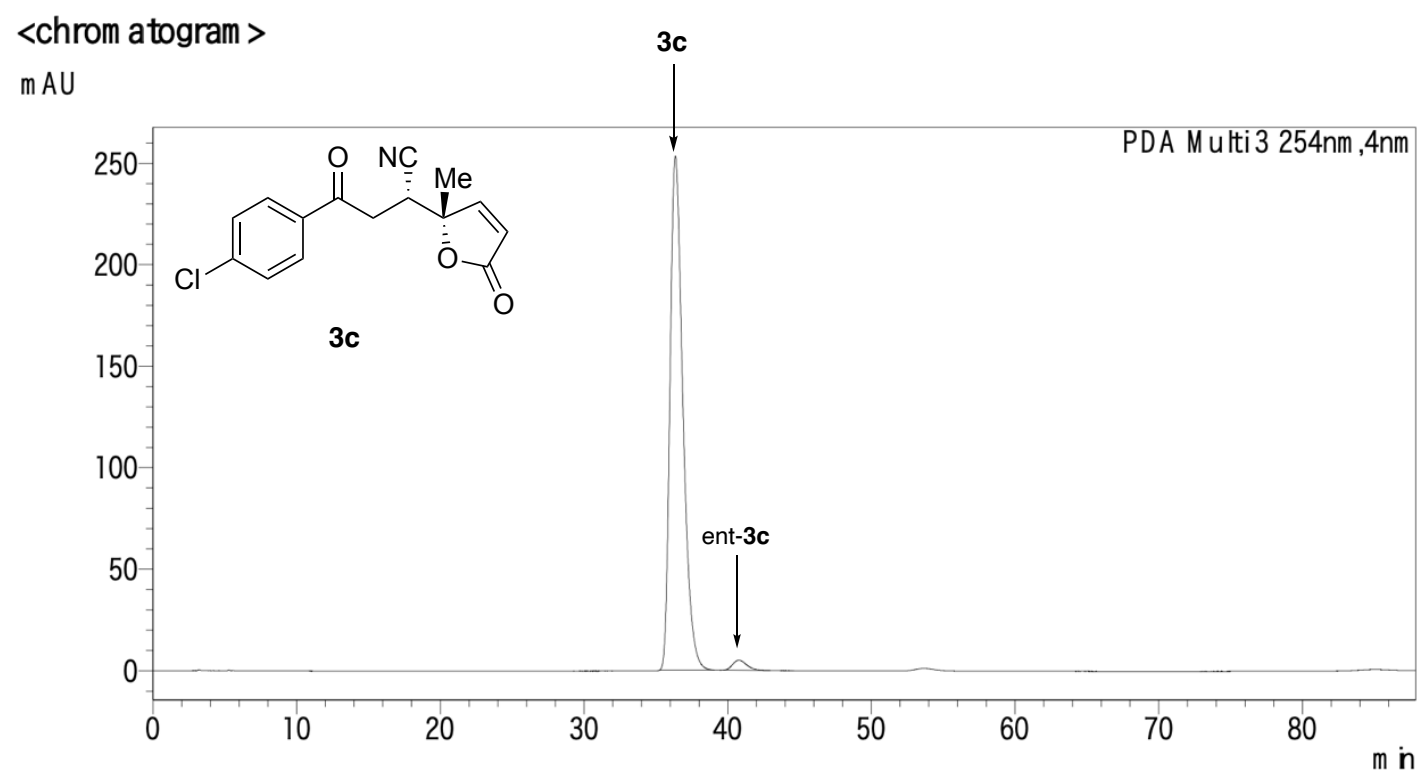

<peak report

PDA Ch3 254nm

\begin{tabular}{|r|r|r|}
\hline peak\# & retention tim e & area\% \\
\hline 1 & 36.352 & 97.819 \\
\hline 2 & 40.773 & 2.181 \\
\hline
\end{tabular}




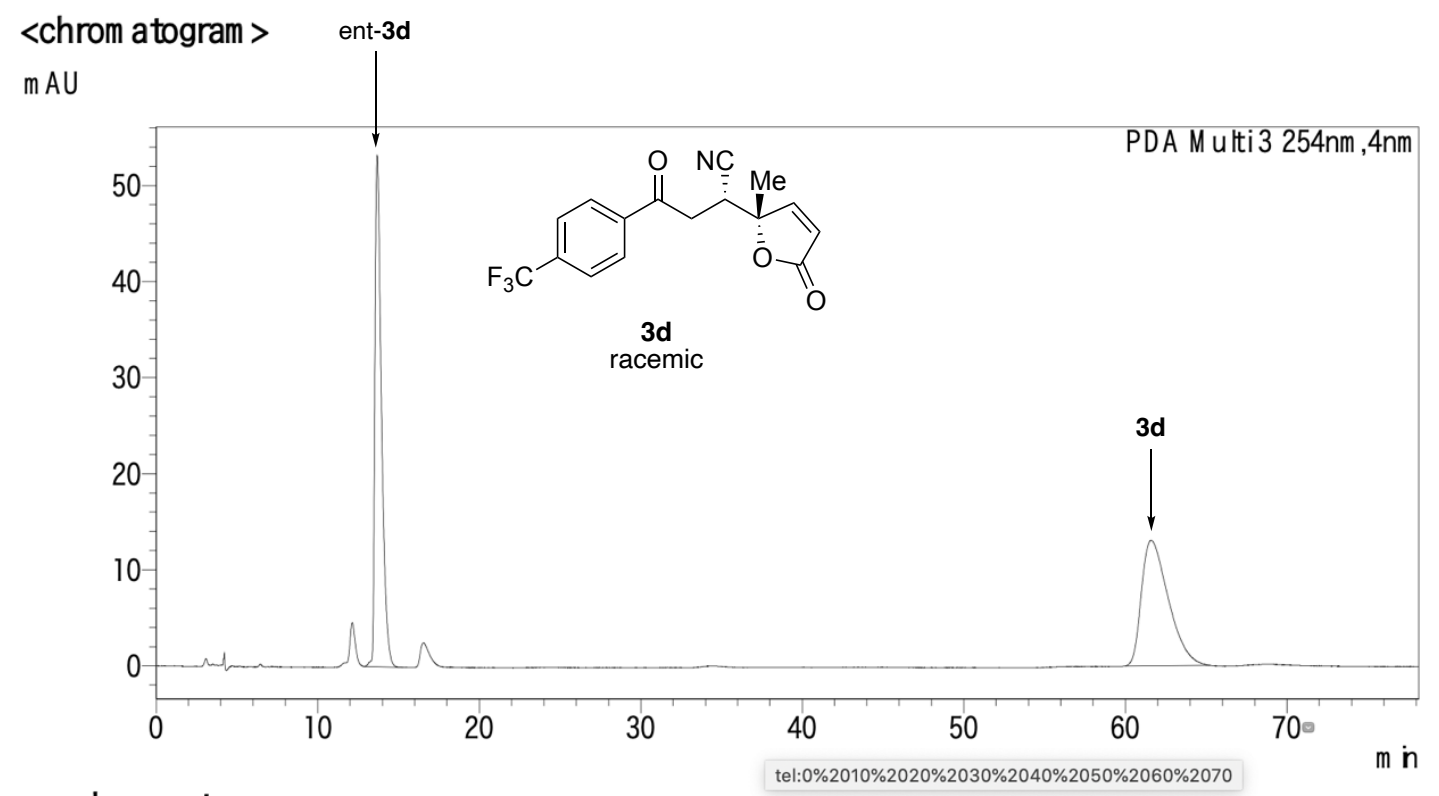

<peak report

PDA Ch3 254nm

\begin{tabular}{|r|r|r|}
\hline peak\# & retention tm e & area\% \\
\hline 1 & 13.680 & 50.036 \\
\hline 2 & 61.601 & 49.964 \\
\hline
\end{tabular}

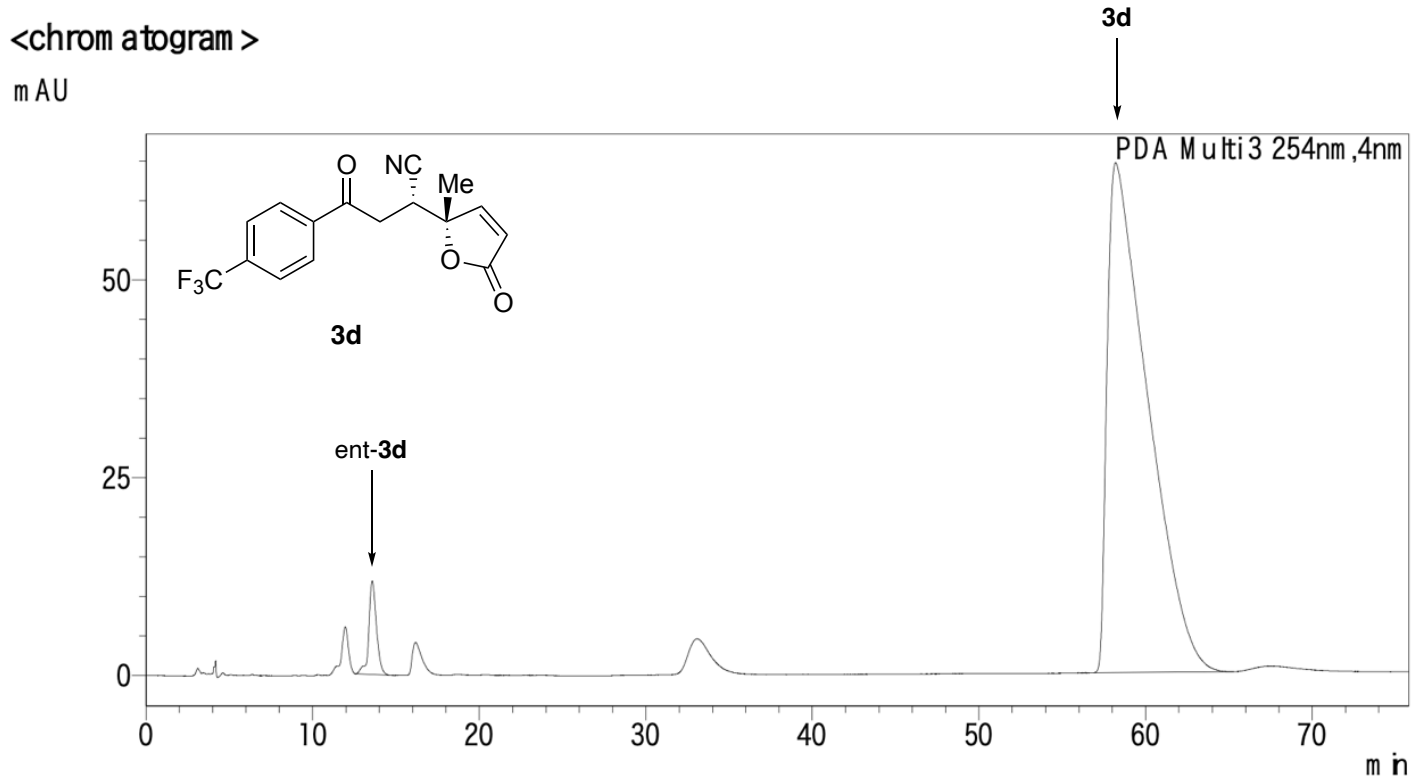

$<$ peak report

PDA Ch3 254nm

\begin{tabular}{|r|r|r|}
\hline peak\# & retention tim e & area\% \\
\hline 1 & 13.580 & 3.377 \\
\hline 2 & 58.206 & 96.623 \\
\hline
\end{tabular}




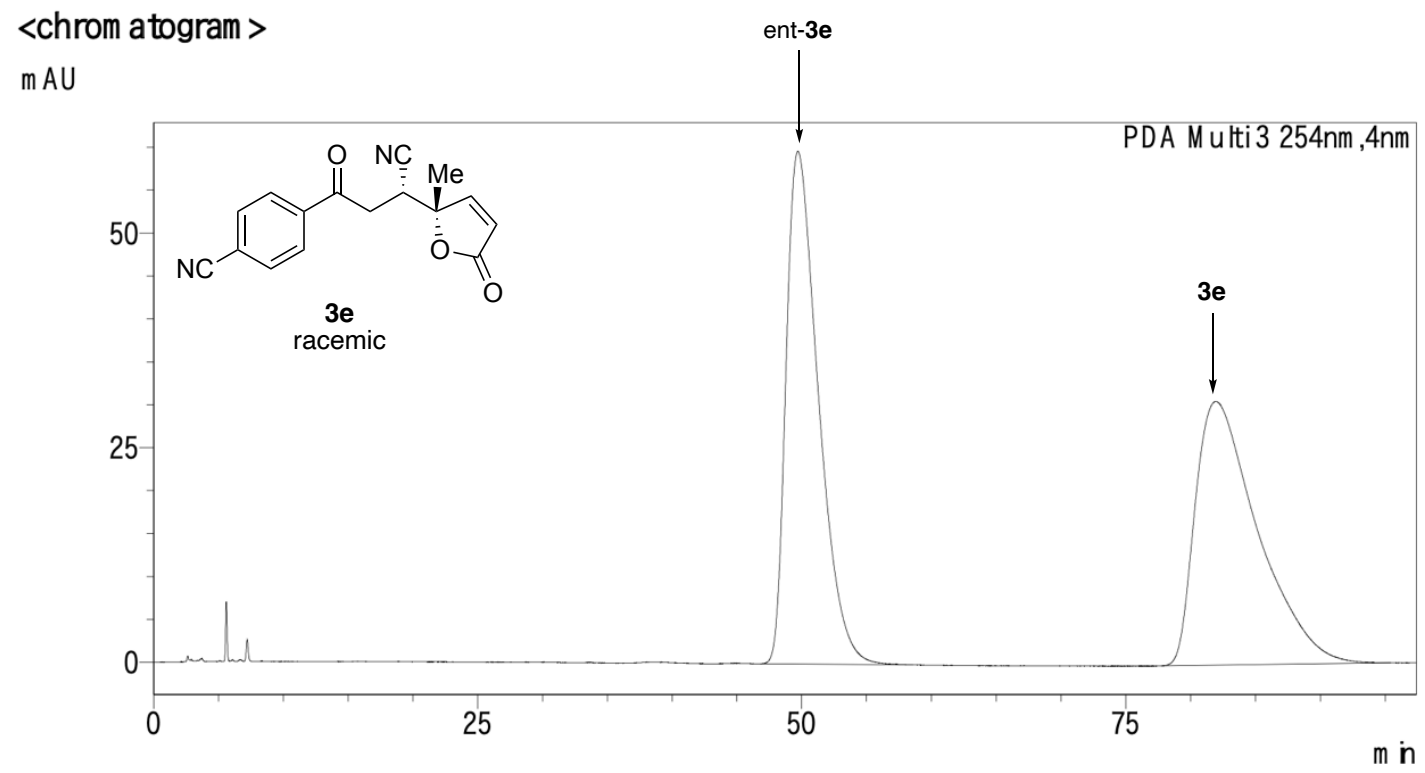

<peak report

PDA Ch3 254nm

\begin{tabular}{|r|r|r|}
\hline peak\# & retention time & \multicolumn{2}{c|}{ area\% } \\
\hline 1 & 49.710 & 50.129 \\
\hline 2 & 81.951 & 49.871 \\
\hline
\end{tabular}

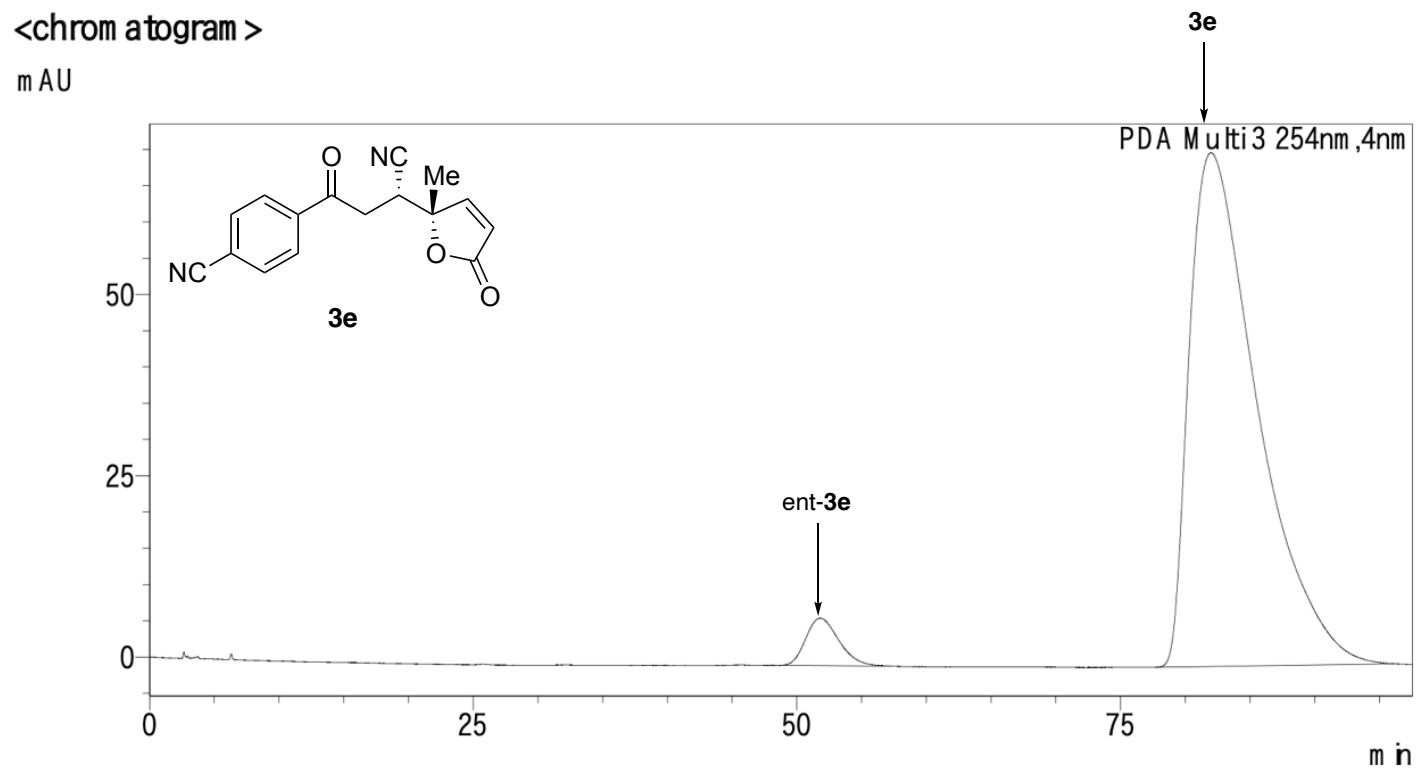

$<$ peak report

PDA Ch3 254nm

\begin{tabular}{|r|r|r|}
\hline peak\# & retention time & area\% \\
\hline 1 & 51.843 & 4.318 \\
\hline 2 & 82.004 & 95.682 \\
\hline
\end{tabular} 


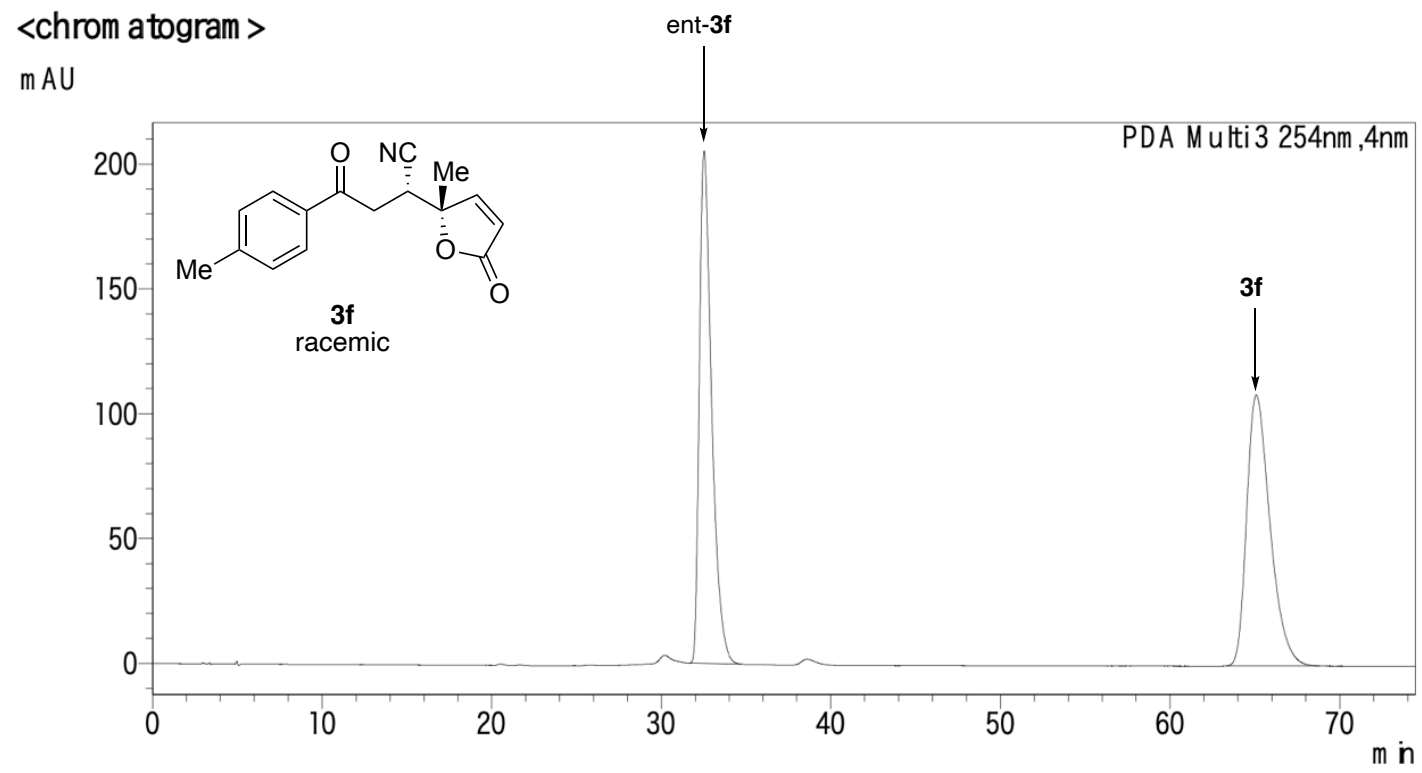

<peak report

PDA Ch3 254nm

\begin{tabular}{|r|r|r|}
\hline peak\# & retention time & \multicolumn{2}{c|}{ area\% } \\
\hline 1 & 32.515 & 49.928 \\
\hline 2 & 65.083 & 50.072 \\
\hline
\end{tabular}

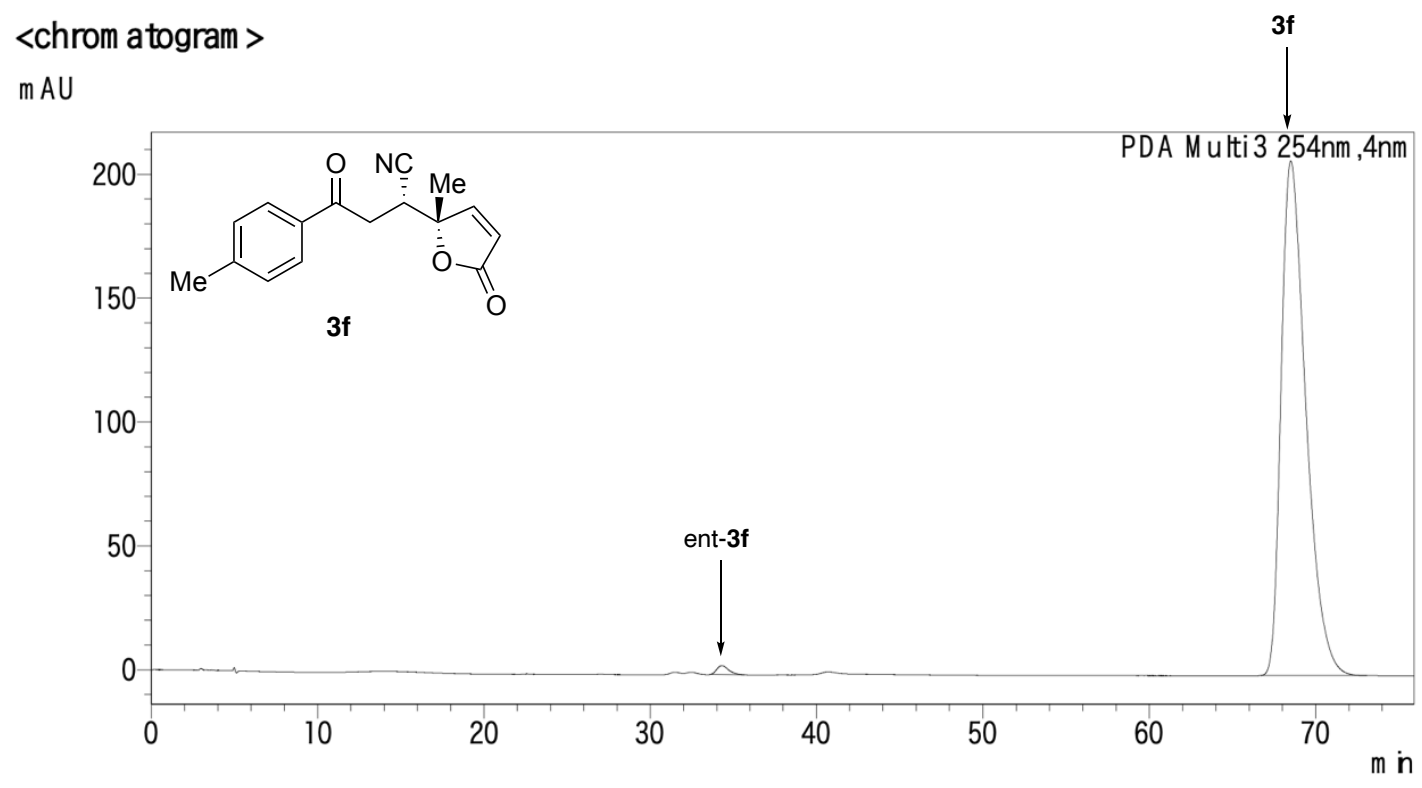

$<$ peak report

PDA Ch3 254nm

\begin{tabular}{|r|r|r|}
\hline peak\# & retention time & \multicolumn{2}{c|}{ area\% } \\
\hline 1 & 34.295 & 0.869 \\
\hline 2 & 68.507 & 99.131 \\
\hline
\end{tabular} 


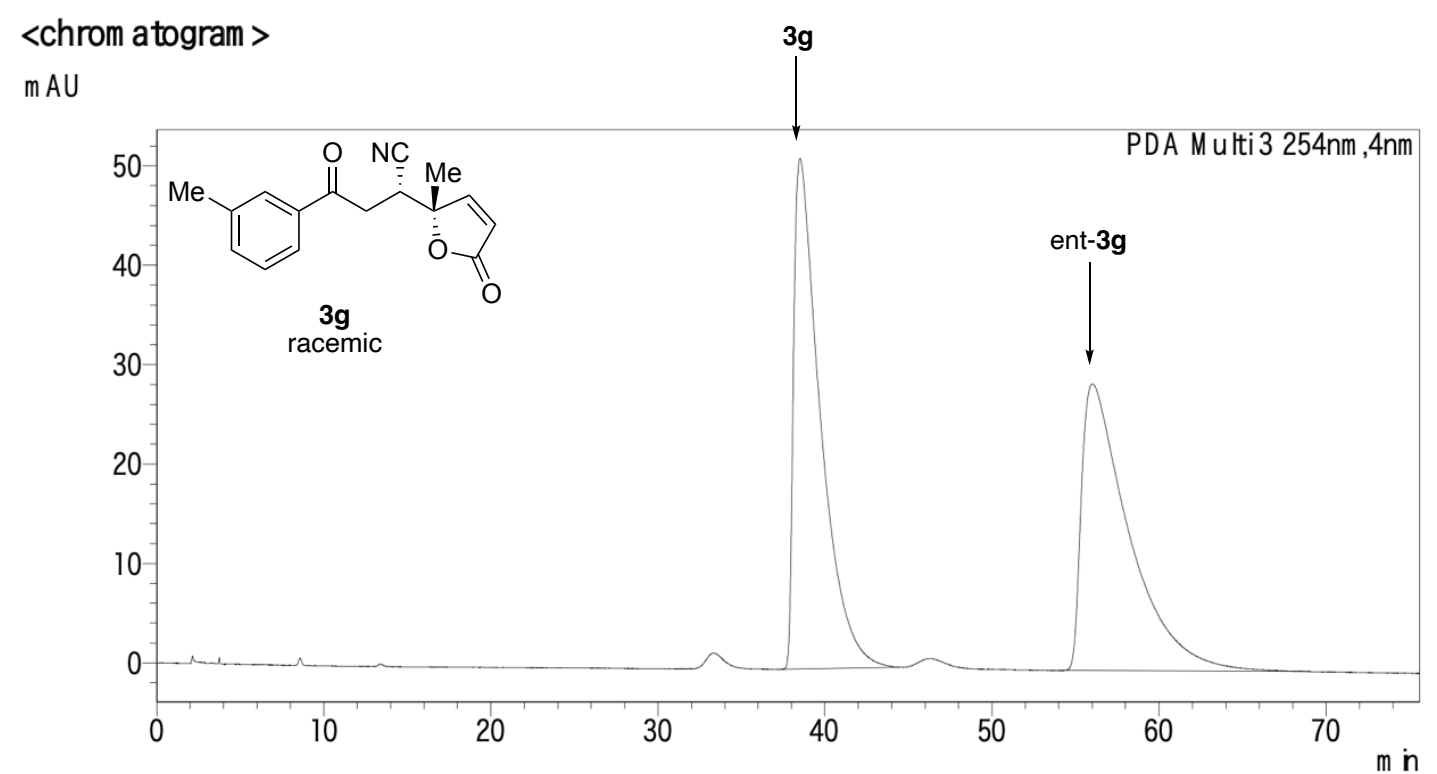

<peak report

PDA Ch3 254nm

\begin{tabular}{|r|r|r|}
\hline peak\# & retention tim e & area\% \\
\hline 1 & 38.520 & 49.995 \\
\hline 2 & 56.002 & 50.005 \\
\hline
\end{tabular}

<chrom atogram >

$\mathrm{mAU}$

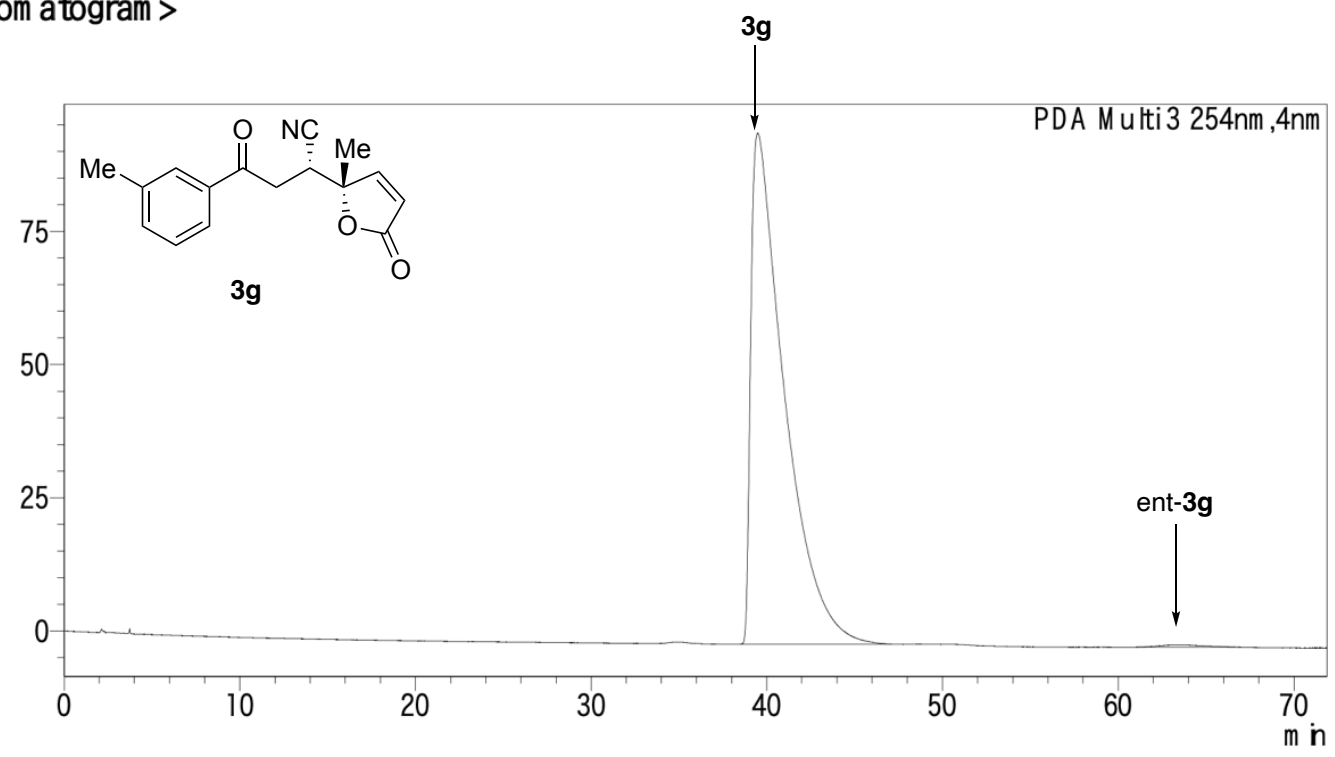

$<$ peak report

PDA Ch3 254nm

\begin{tabular}{|r|r|r|}
\hline peak\# & retention tim e & area\% \\
\hline 1 & 39.489 & 99.421 \\
\hline 2 & 63.479 & 0.579 \\
\hline
\end{tabular}




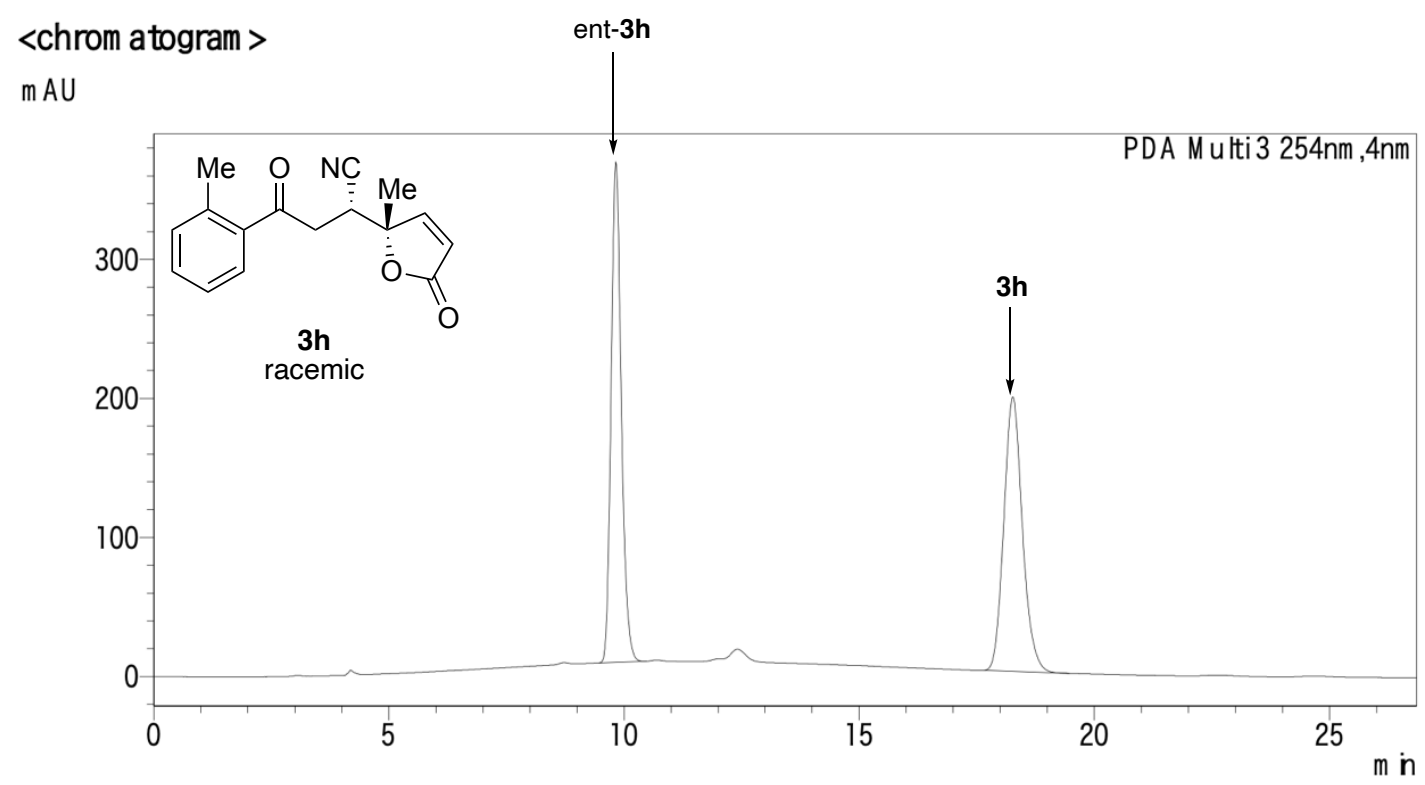

<peak report

PDA Ch3 254nm

\begin{tabular}{|r|r|r|}
\hline peak\# & retention tim e & area\% \\
\hline 1 & 9.825 & 49.917 \\
\hline 2 & 18.269 & 50.083 \\
\hline
\end{tabular}

$<$ chrom atogram >

mAU

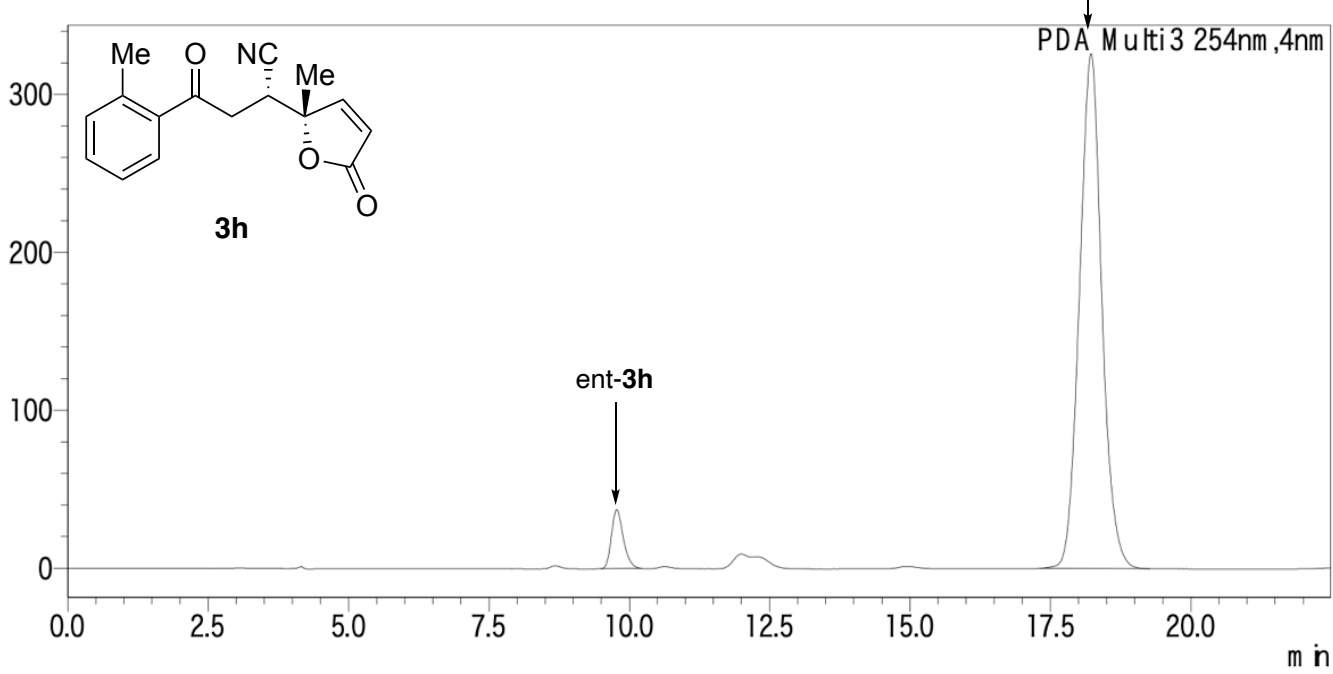

<peak report

PDA Ch3 254nm

\begin{tabular}{|r|r|r|}
\hline peak\# & retention time & area\% \\
\hline 1 & 9.773 & 5.731 \\
\hline 2 & 18.224 & 94.269 \\
\hline
\end{tabular}




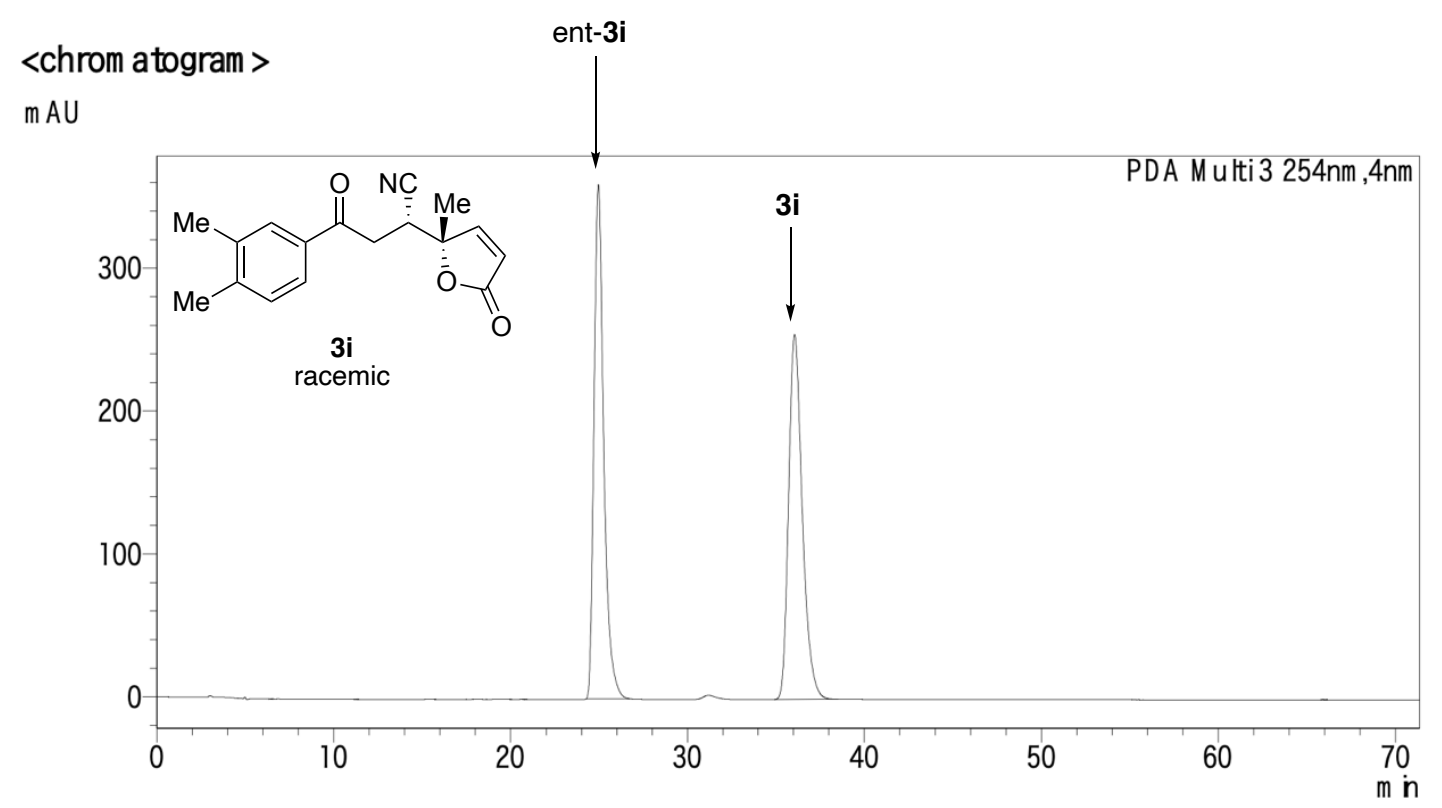

$<$ peak report

PDA Ch3 254nm

\begin{tabular}{|r|r|r|}
\hline peak\# & retention tim e & area\% \\
\hline 1 & 24.954 & 50.129 \\
\hline 2 & 36.062 & 49.871 \\
\hline
\end{tabular}

<chrom atogram >

$\mathrm{mAU}$

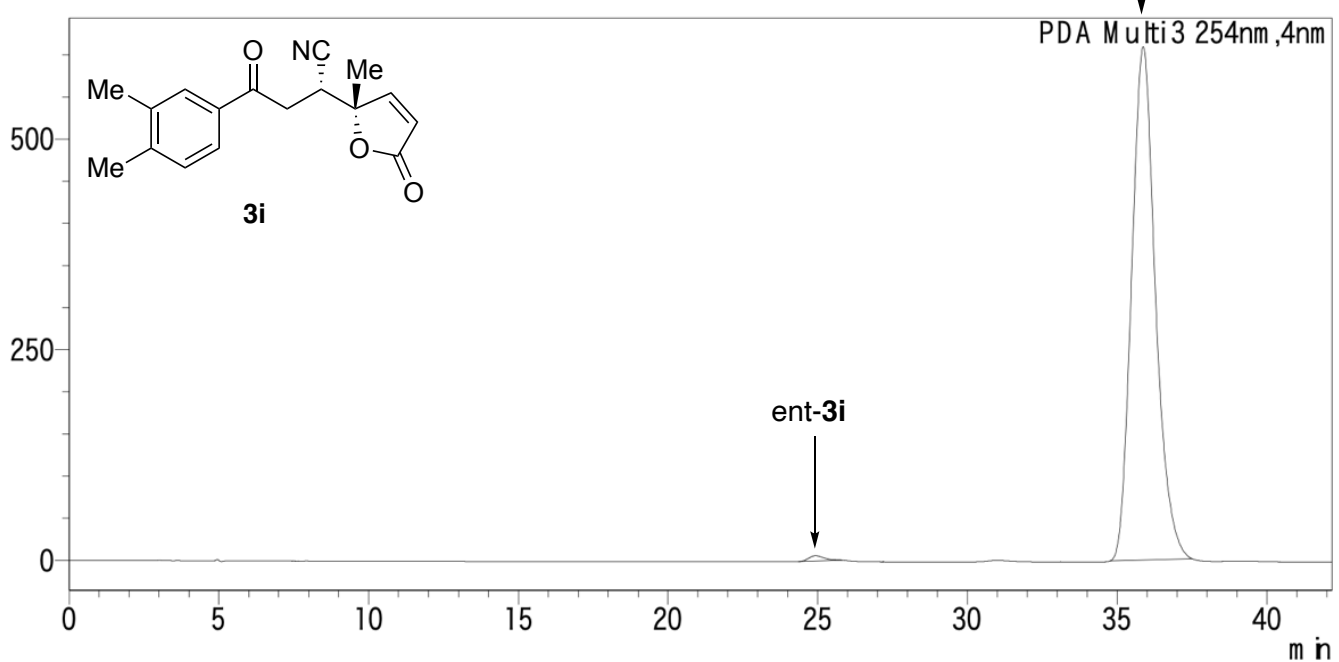

<peak report

PDA Ch3 254nm

\begin{tabular}{|r|r|rr|}
\hline peak\# & retention tim e & area\% & \\
\hline 1 & 24.927 & & 0.688 \\
\hline 2 & 35.869 & & 99.312 \\
\hline
\end{tabular}




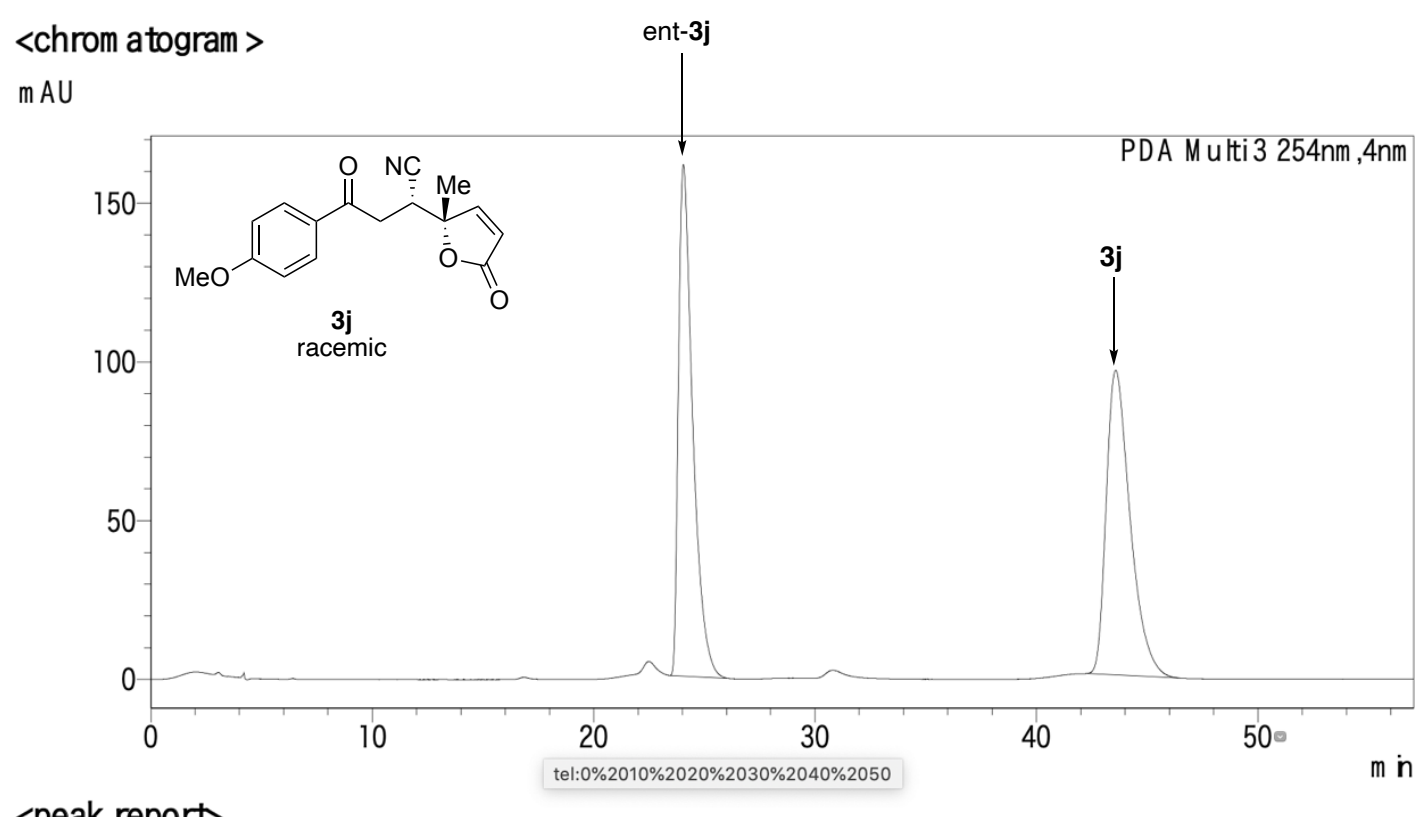

$<$ peak report

PDA Ch3 254nm

\begin{tabular}{|r|r|r|}
\hline peak\# & retention tim e & area\% \\
\hline 1 & 24.046 & \multicolumn{4}{|c|}{49.950} \\
\hline 2 & 43.582 & 50.050 \\
\hline
\end{tabular}

$43.582 \quad 50.050$

<chrom atogram >

3j

$m A U$

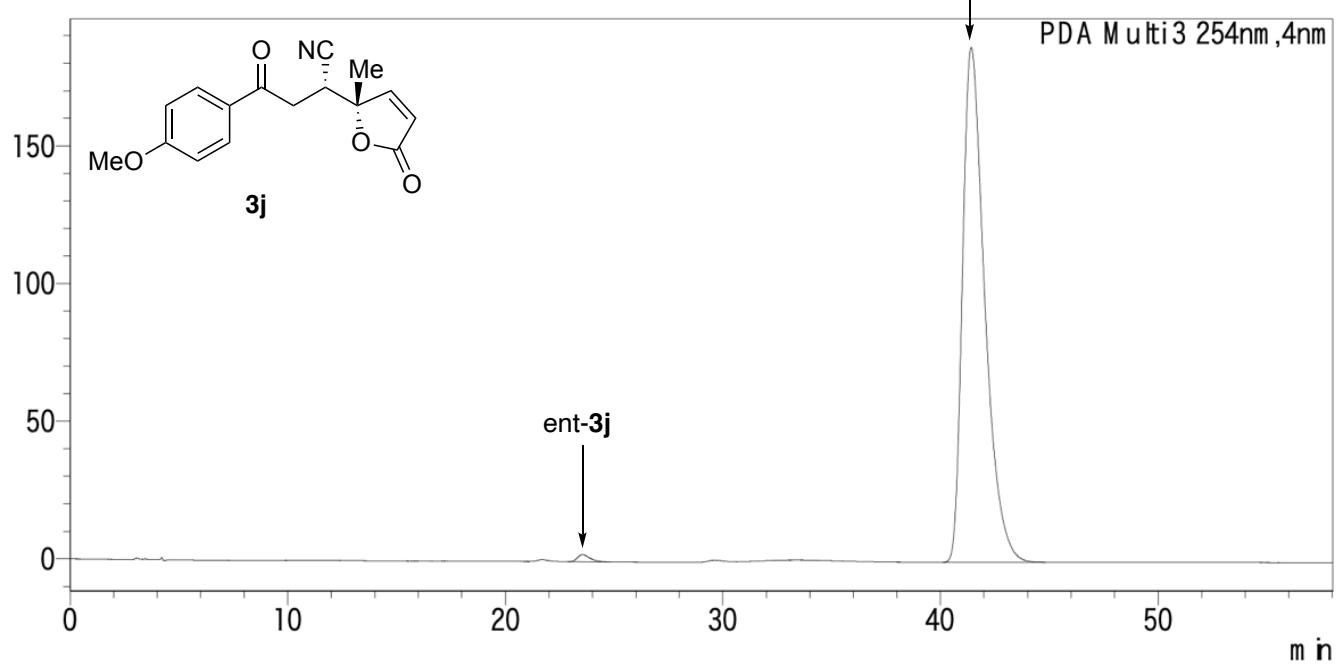

$<$ peak report

PDA Ch3 254nm

\begin{tabular}{|r|r|r|}
\hline peak\# & retention tim e & area\% \\
\hline 1 & 23.561 & 0.776 \\
\hline 2 & 41.418 & 99.224 \\
\hline
\end{tabular}




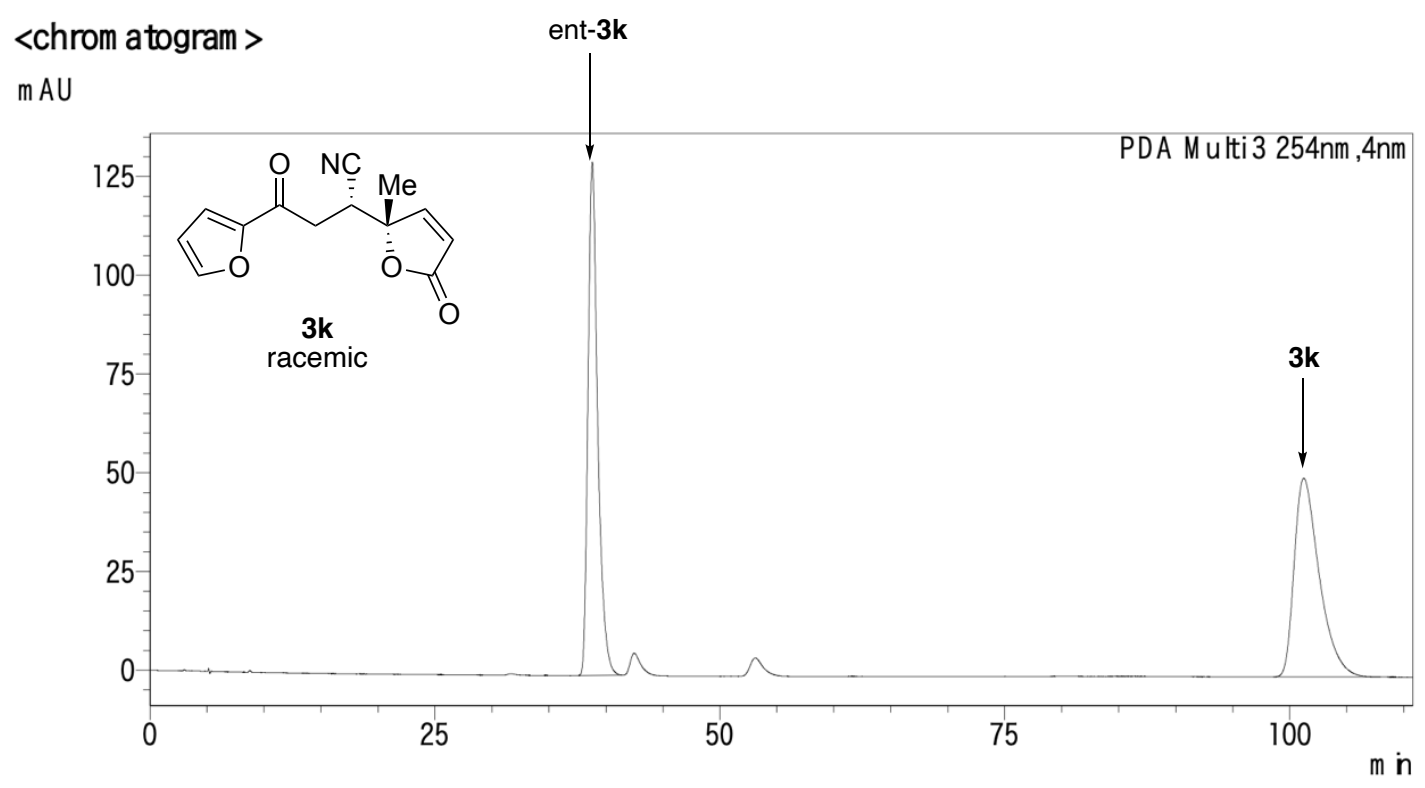

<peak report

PDA Ch3 254nm

\begin{tabular}{|r|r|r|}
\hline peak\# & retention tim e & area\% \\
\hline 1 & 38.798 & 49.943 \\
\hline 2 & 101.240 & 50.057 \\
\hline
\end{tabular}

$<$ chrom atogram >

$\mathrm{mAU}$

3k

PDA M ulti $3254 \mathrm{~nm}, 4 \mathrm{~nm}$

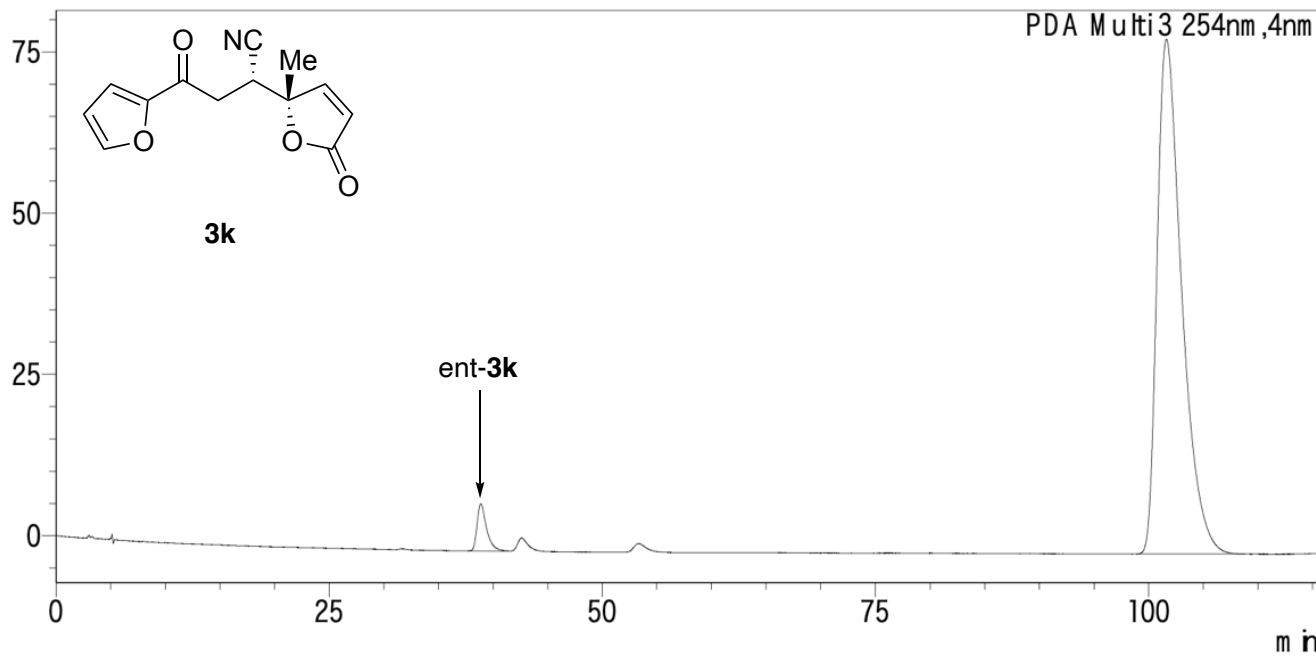

$<$ peak report

PDA Ch3 254nm

\begin{tabular}{|r|r|r|}
\hline peak\# & retention tim e & area\% \\
\hline 1 & 38.879 & 3.421 \\
\hline 2 & 101.630 & 96.579 \\
\hline
\end{tabular}




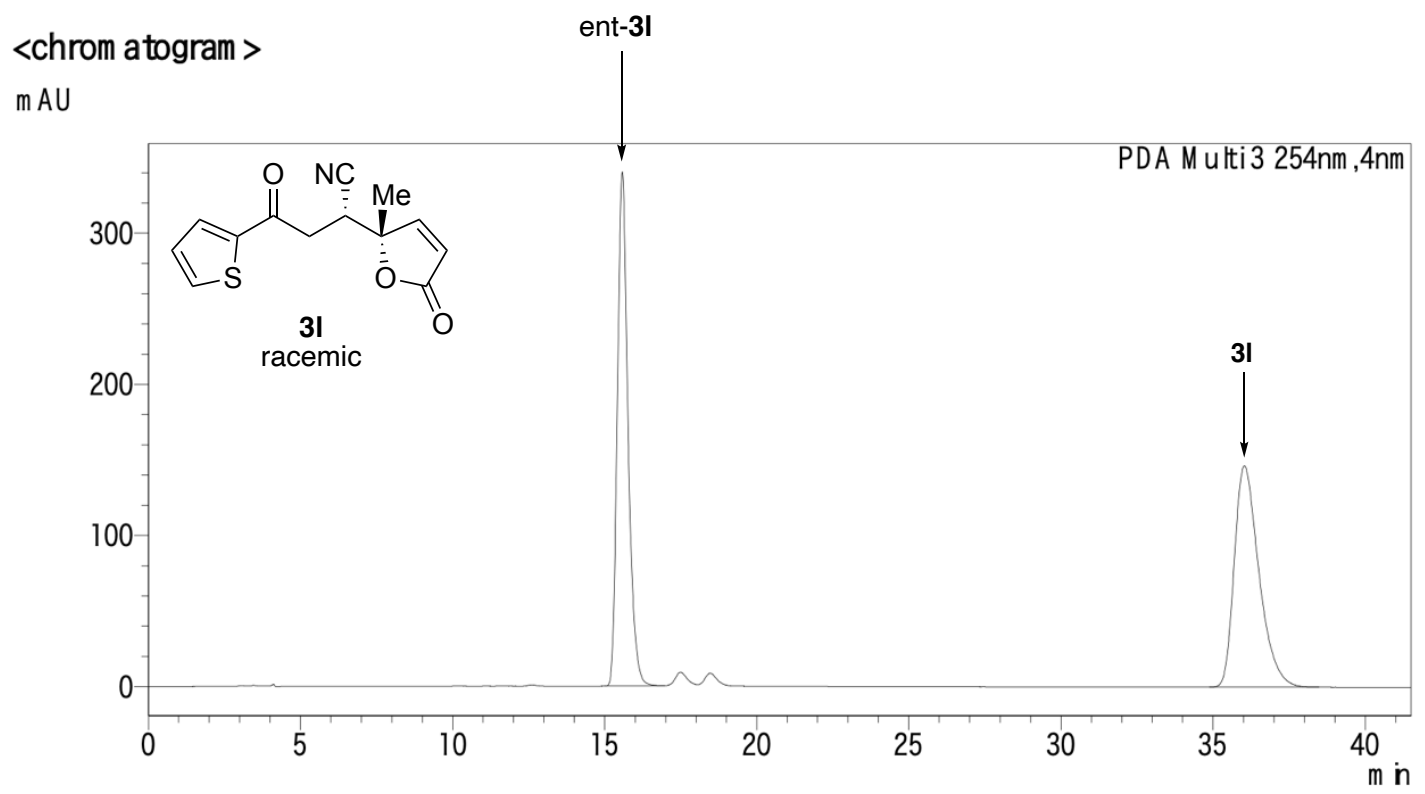

$<$ peak report

PDA Ch3 254nm

\begin{tabular}{|rr|r|}
\hline peak\# & retention tim e & \multicolumn{2}{c|}{ area\% } \\
\hline 1 & 15.575 & 50.131 \\
\hline 2 & 36.026 & 49.869 \\
\hline
\end{tabular}

$<$ chrom atogram >

$\mathrm{mAU}$

3I

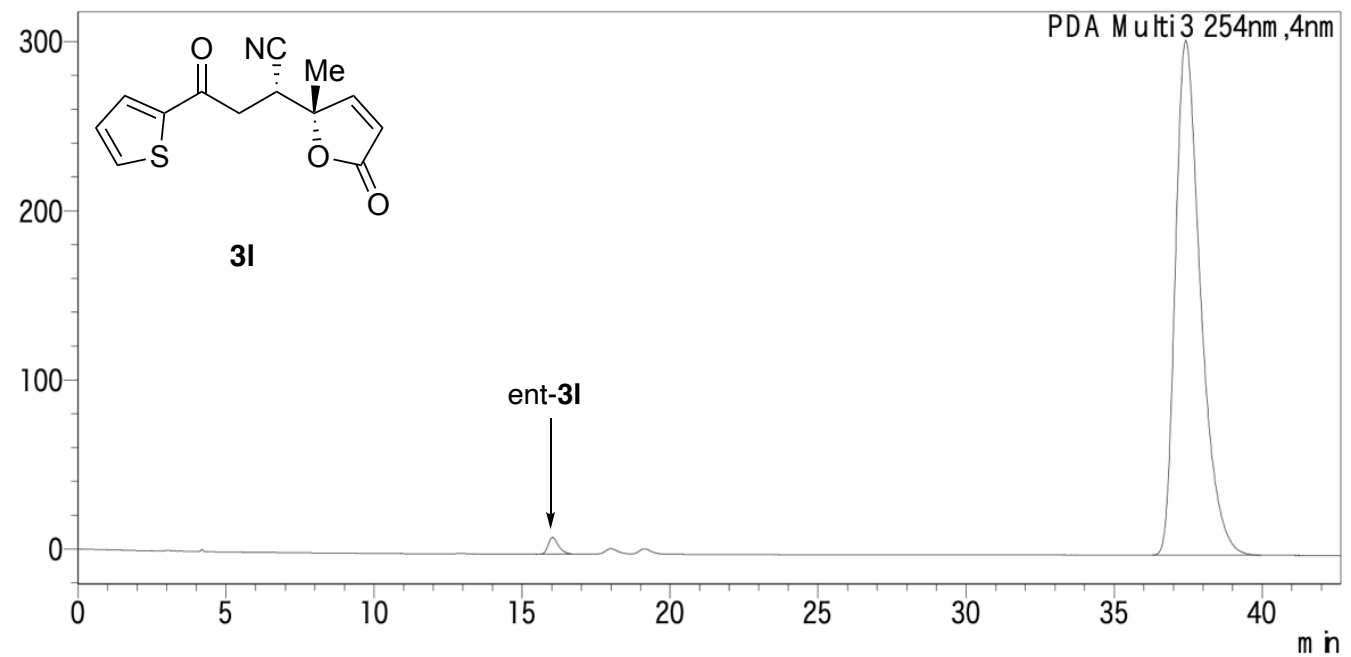

$<$ peak report

PDA Ch3 254nm

\begin{tabular}{|r|r|r|}
\hline peak\# & retention tim e & area\% \\
\hline 1 & 16.025 & 1.313 \\
\hline 2 & 37.417 & 98.687 \\
\hline
\end{tabular}




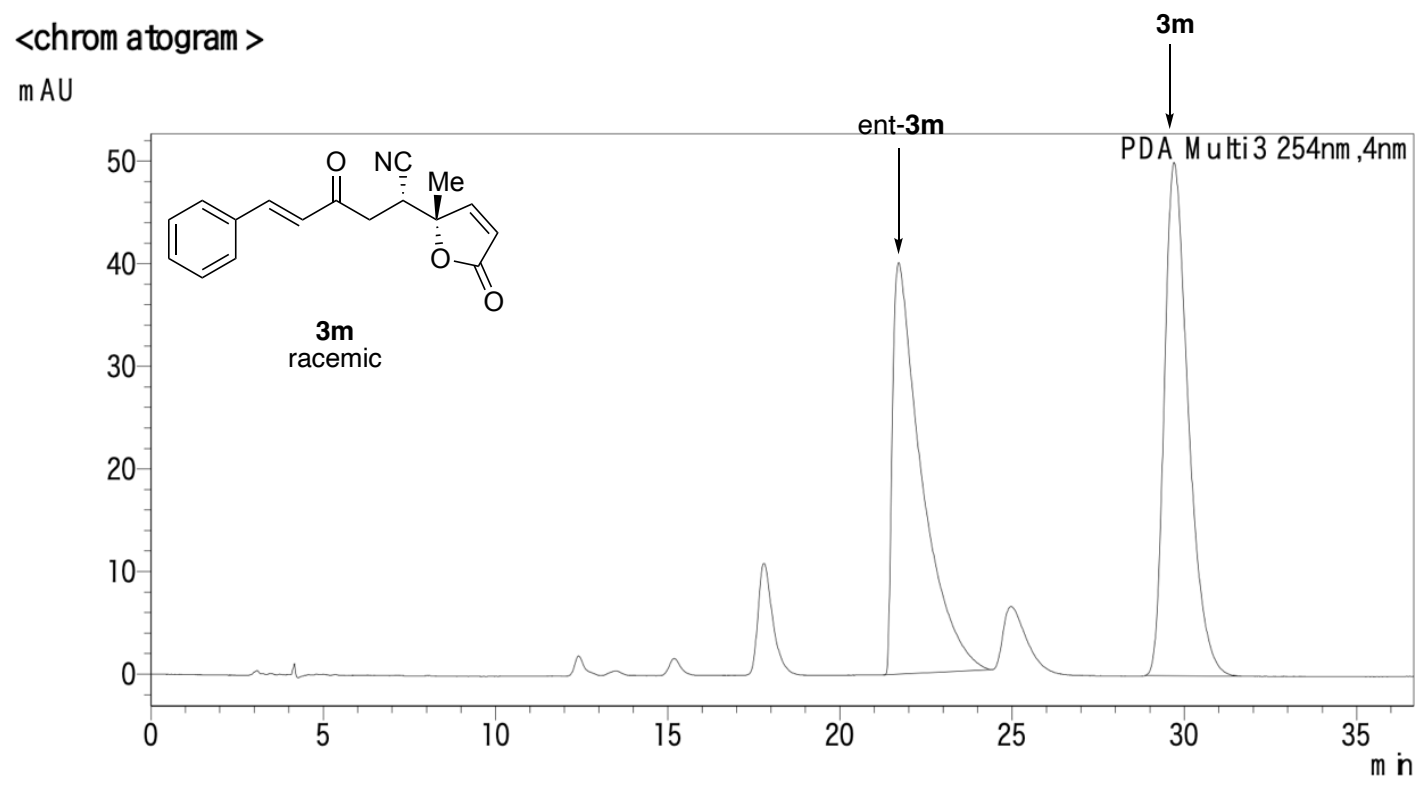

<peak report

PDA Ch3 254nm

\begin{tabular}{|r|r|r|}
\hline peak\# & retention tim e & area\% \\
\hline 1 & 21.708 & 49.377 \\
\hline 2 & 29.700 & 50.623 \\
\hline
\end{tabular}

<chrom atogram >

$\mathrm{mAU}$

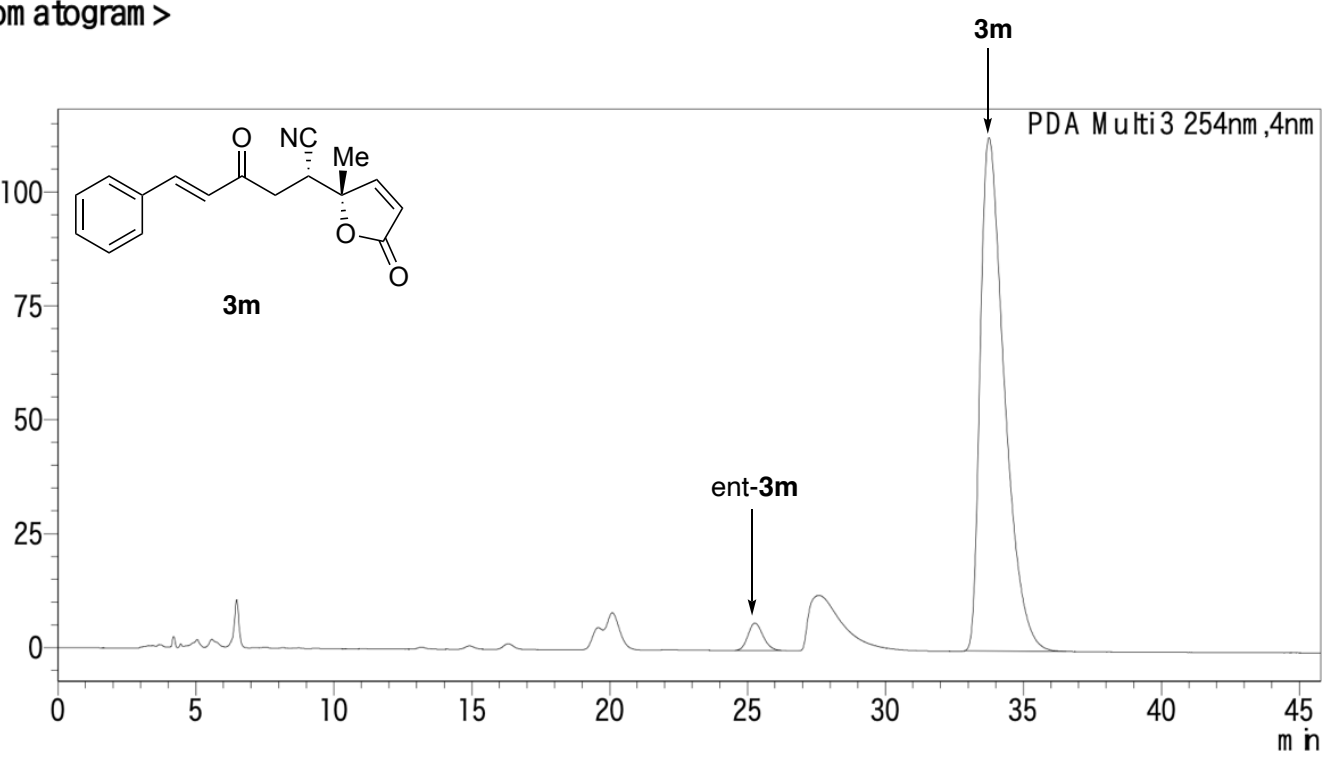

<peak report

\begin{tabular}{|r|r|r|}
\begin{tabular}{|l} 
PDA Ch3 254nm \\
peak\#
\end{tabular} & retention time & area\% \\
\hline 1 & 25.276 & 3.190 \\
\hline 2 & 33.754 & \\
\hline
\end{tabular}




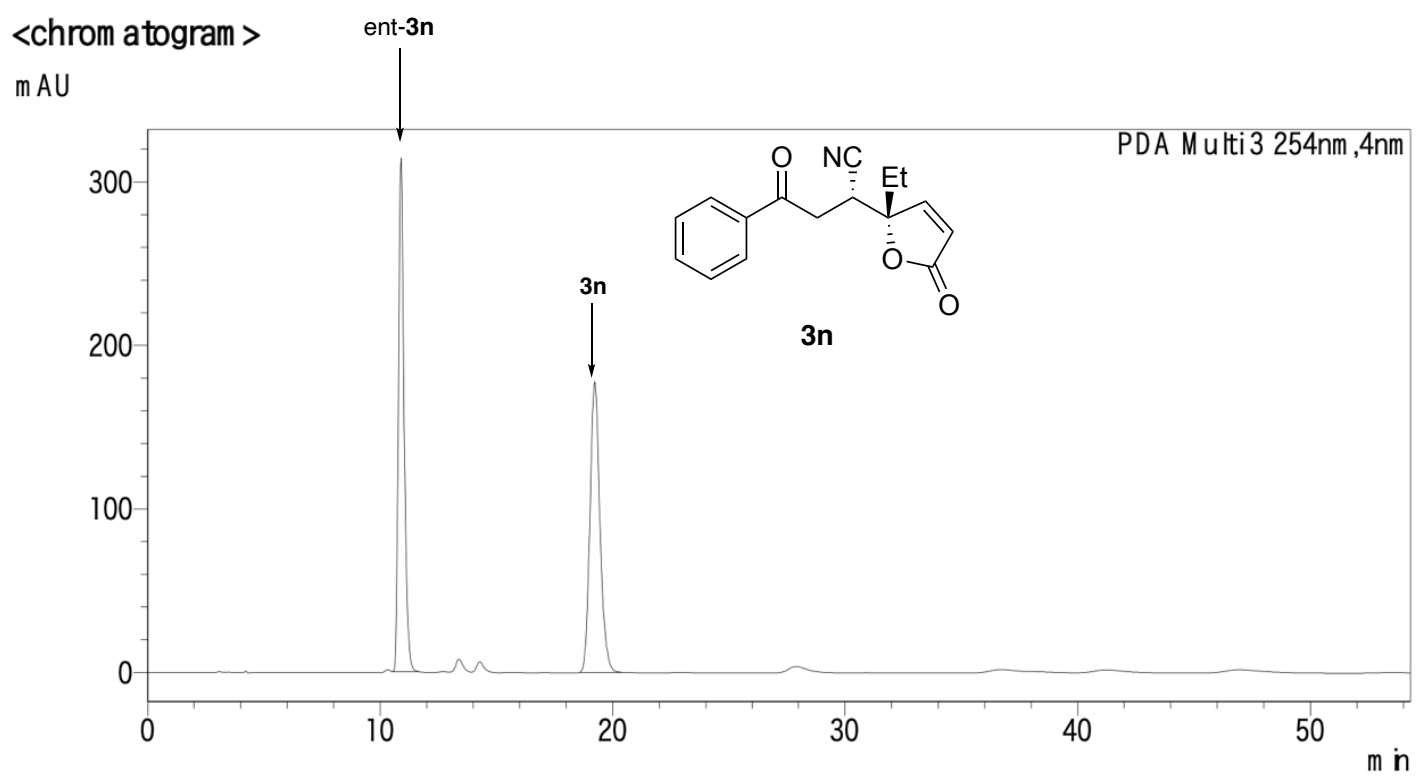

$<$ peak report

PDA Ch3 254nm

\begin{tabular}{|r|r|r|}
\hline peak\# & retention tim e & \multicolumn{2}{c|}{ area\% } \\
\hline 1 & 10.893 & \\
\hline 2 & 19.224 & \\
\hline
\end{tabular}

<chrom atogram >

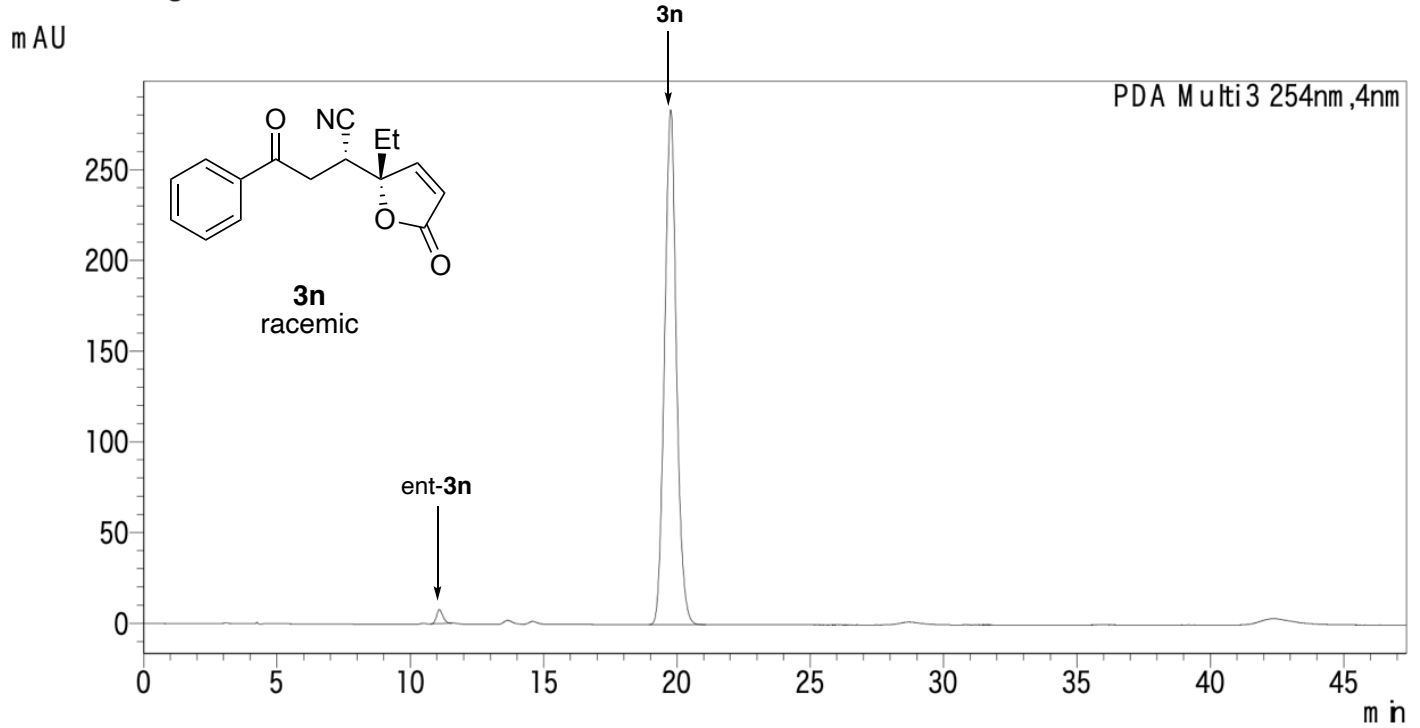

$<$ peak report

PDA Ch3 254nm

\begin{tabular}{|r|r|r|}
\hline peak\# & retention tim e & \multicolumn{2}{c|}{ area\% } \\
\hline 1 & 11.089 & 1.434 \\
\hline 2 & 19.760 & 98.566 \\
\hline
\end{tabular}




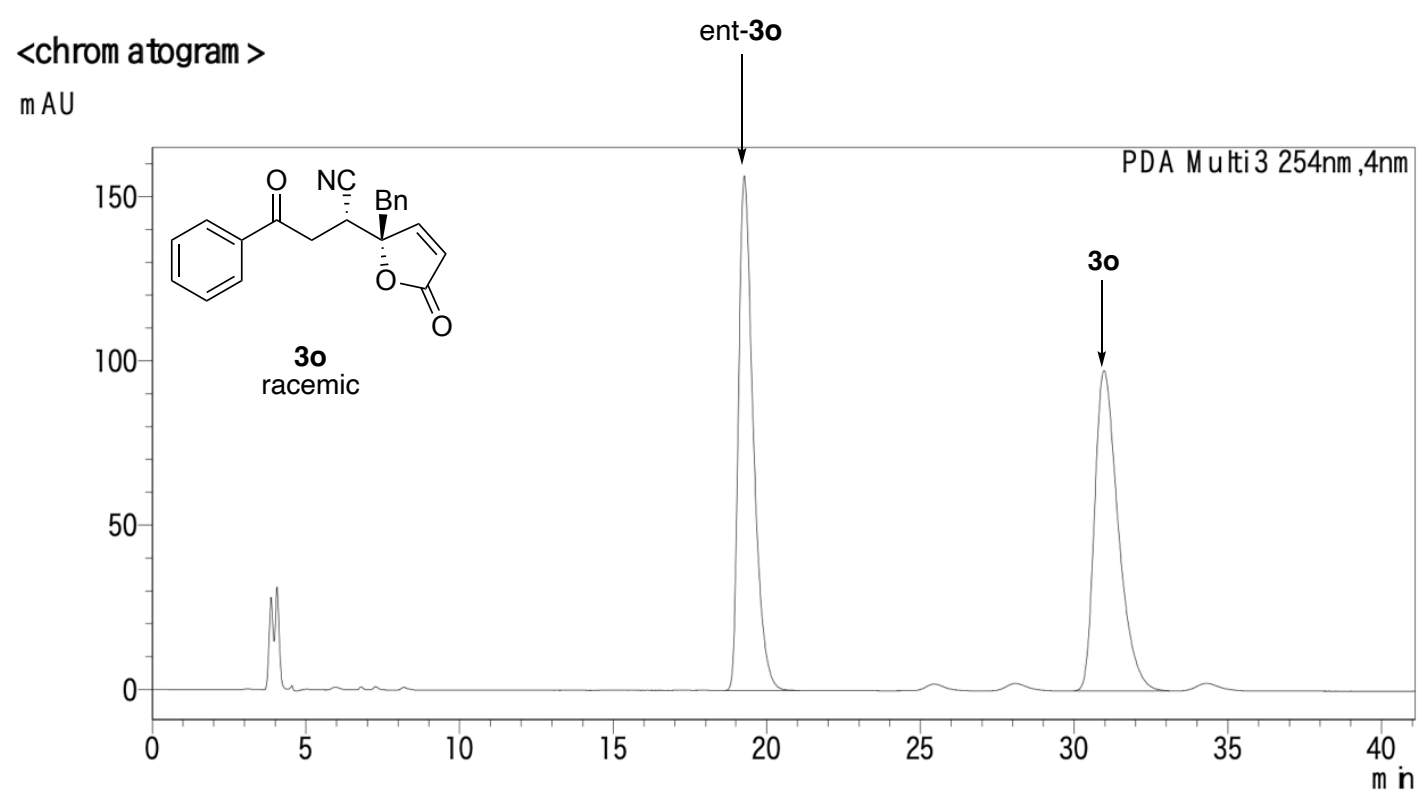

<peak report

PDA Ch3 254nm

\begin{tabular}{|r|r|r|}
\hline peak\# & retention tim e & \multicolumn{2}{c|}{ area\% } \\
\hline 1 & 19.273 & 50.066 \\
\hline 2 & 30.977 & \\
\hline
\end{tabular}

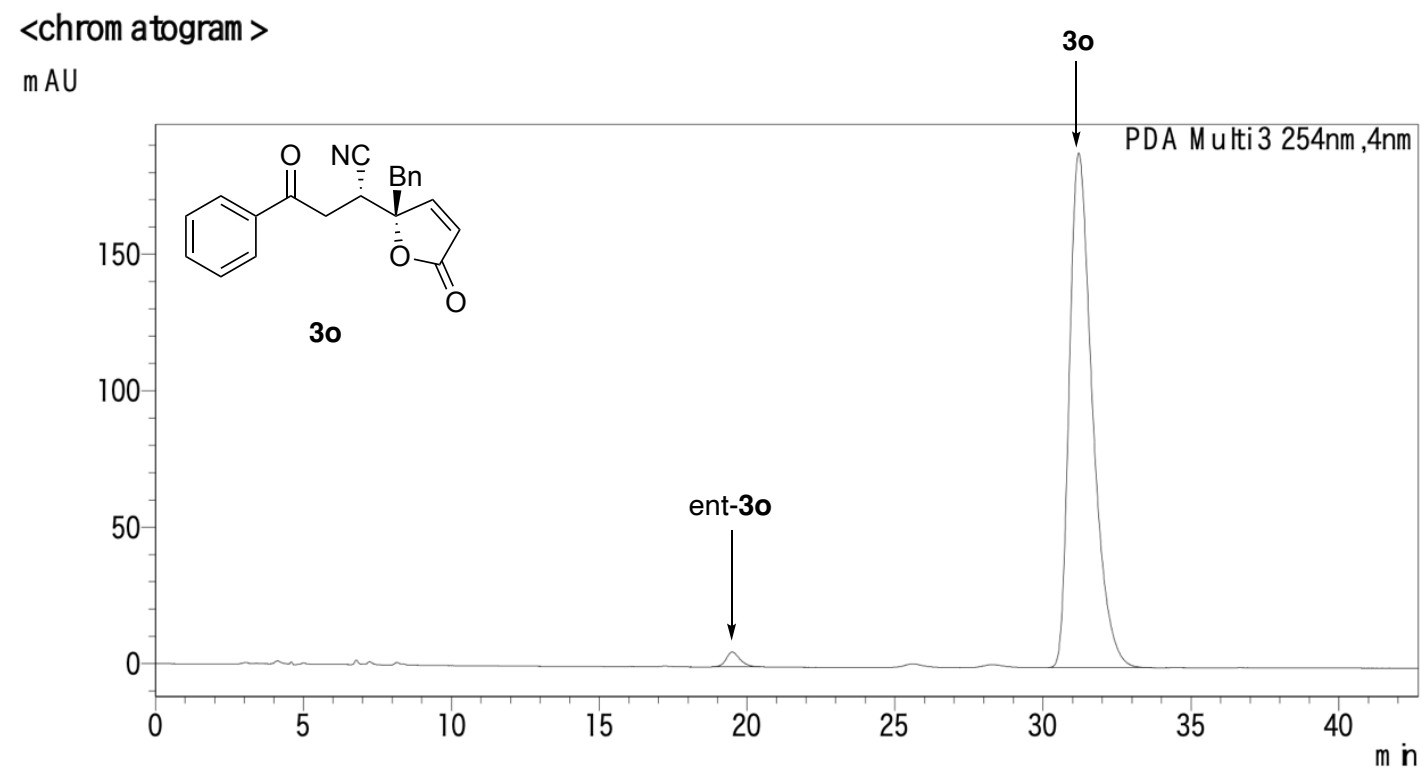

<peak report

PDA Ch3 254nm

\begin{tabular}{|r|r|r|}
\hline peak\# & retention tim e & area\% \\
\hline 1 & 19.489 & 1.762 \\
\hline 2 & 31.209 & 98.238 \\
\hline
\end{tabular}




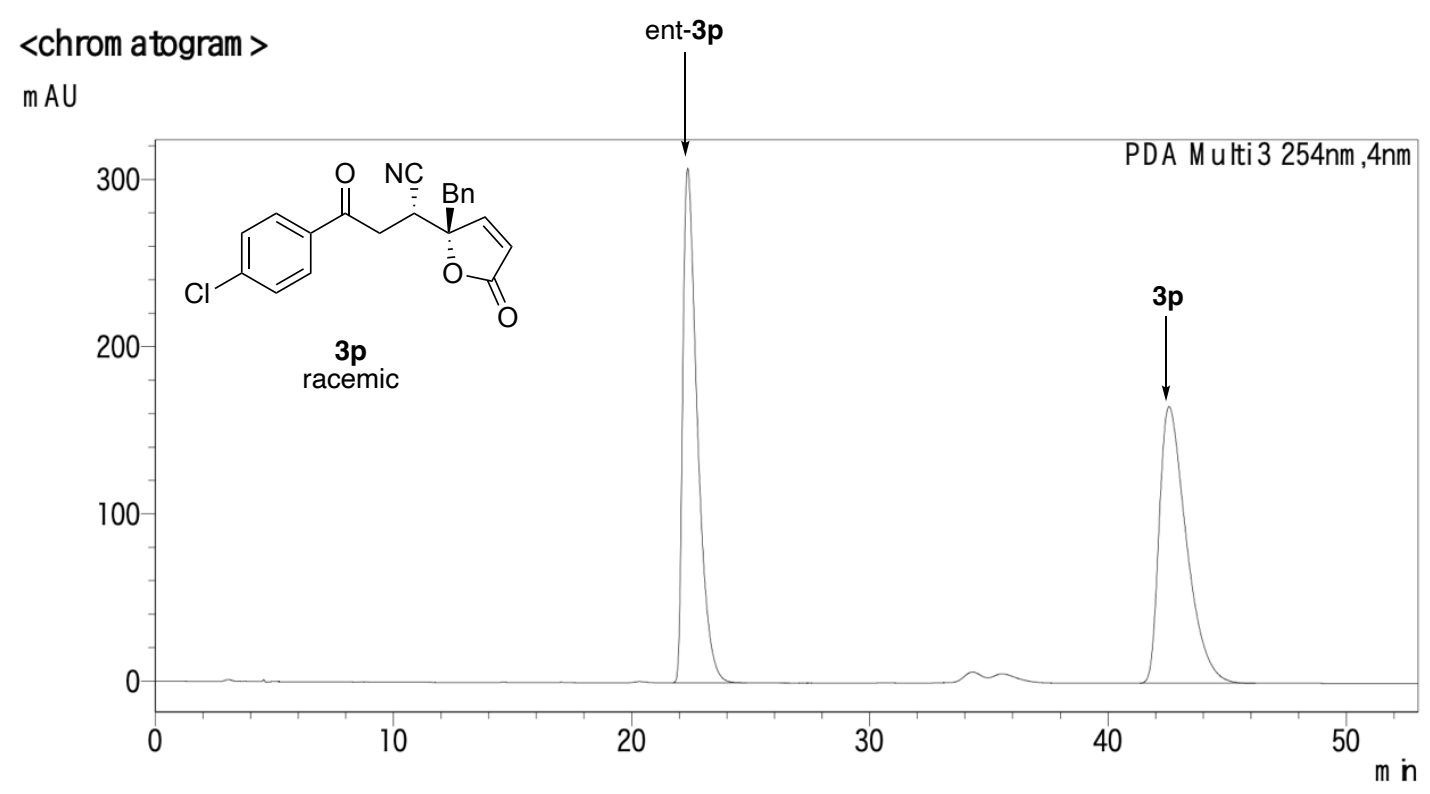

<peak report

PDA Ch3 254nm

\begin{tabular}{|r|r|r|}
\hline peak\# & retention tim e & area\% \\
\hline 1 & 22.347 & \\
\hline 2 & 42.561 & 59.983 \\
\hline
\end{tabular}

<chrom atogram >

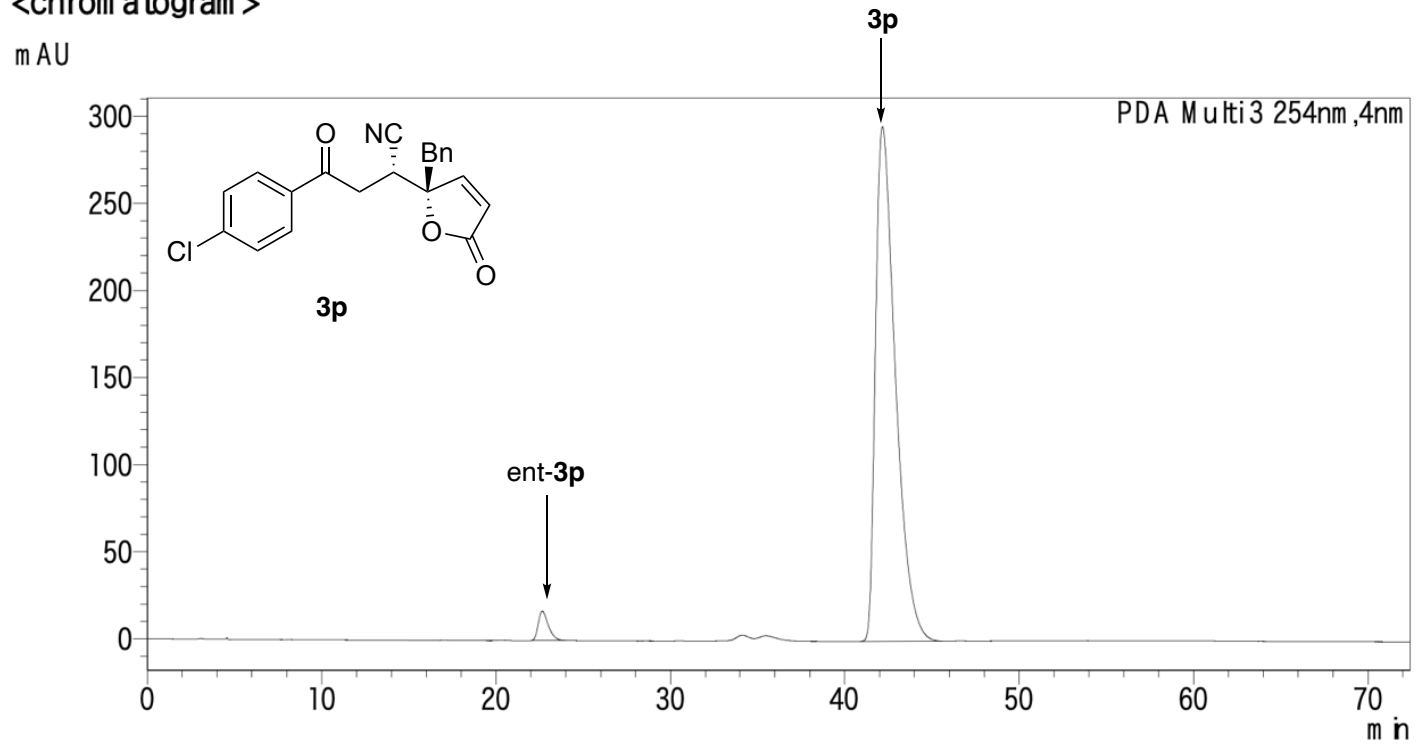

<peak report

PDA Ch3 254nm

\begin{tabular}{|r|r|r|}
\hline peak\# & retention tim e & area\% \\
\hline 1 & 22.647 & 2.828 \\
\hline 2 & 42.171 & 97.172 \\
\hline
\end{tabular} 


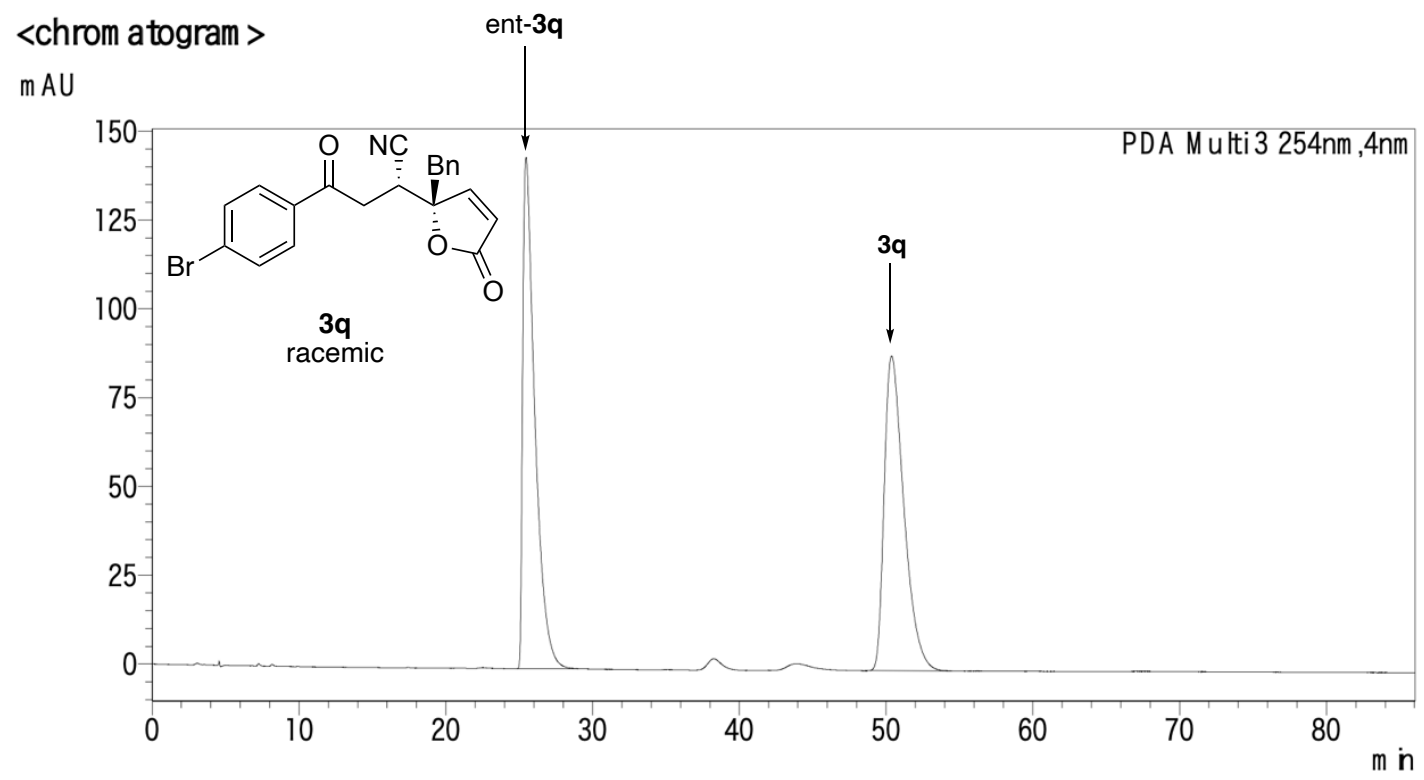

<peak report

PDA Ch3 254nm

\begin{tabular}{|r|r|r|}
\hline peak\# & retention tim e & area\% \\
\hline 1 & 25.466 & 49.980 \\
\hline 2 & 50.386 & 50.020 \\
\hline
\end{tabular}

<chrom atogram >

$\mathrm{mAU}$

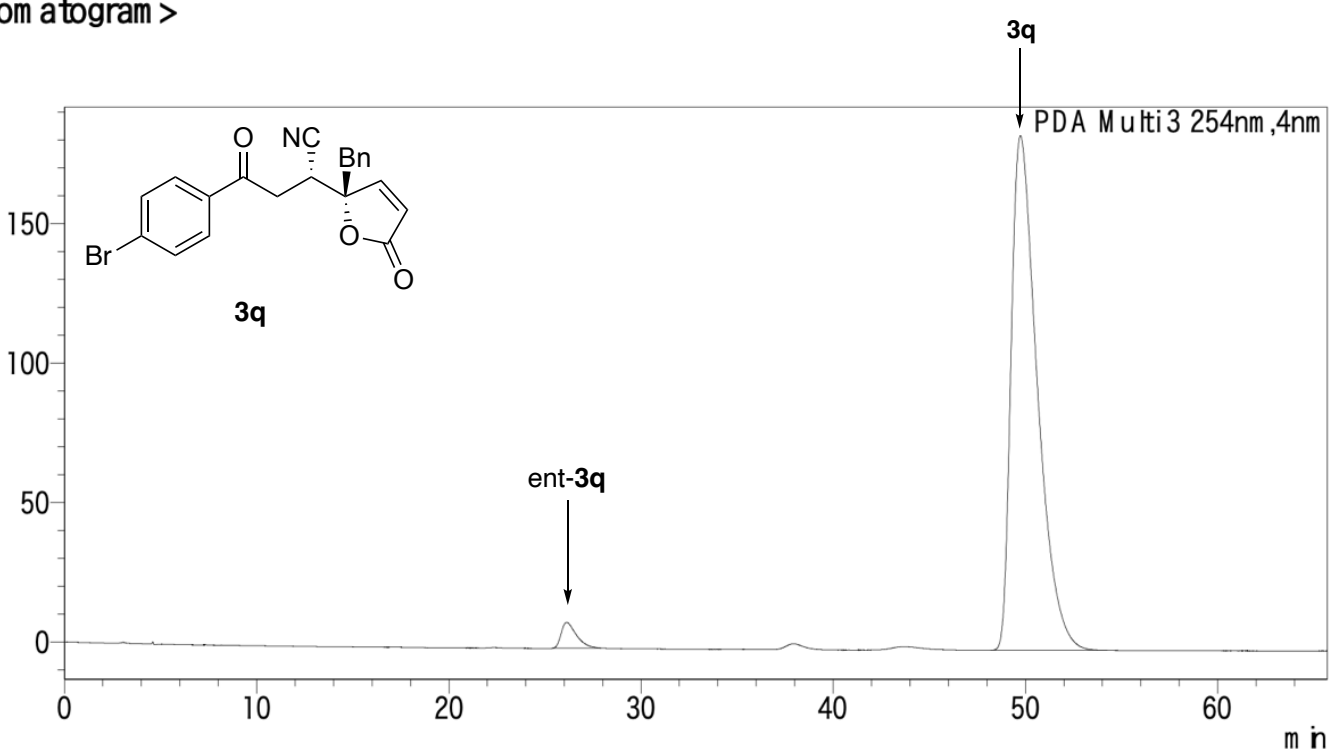

$<$ peak report

PDA Ch3 254nm

\begin{tabular}{|r|r|r|}
\hline peak\# & retention tm e & area\% \\
\hline 1 & 26.123 & 2.818 \\
\hline 2 & 49.744 & 97.182 \\
\hline
\end{tabular}




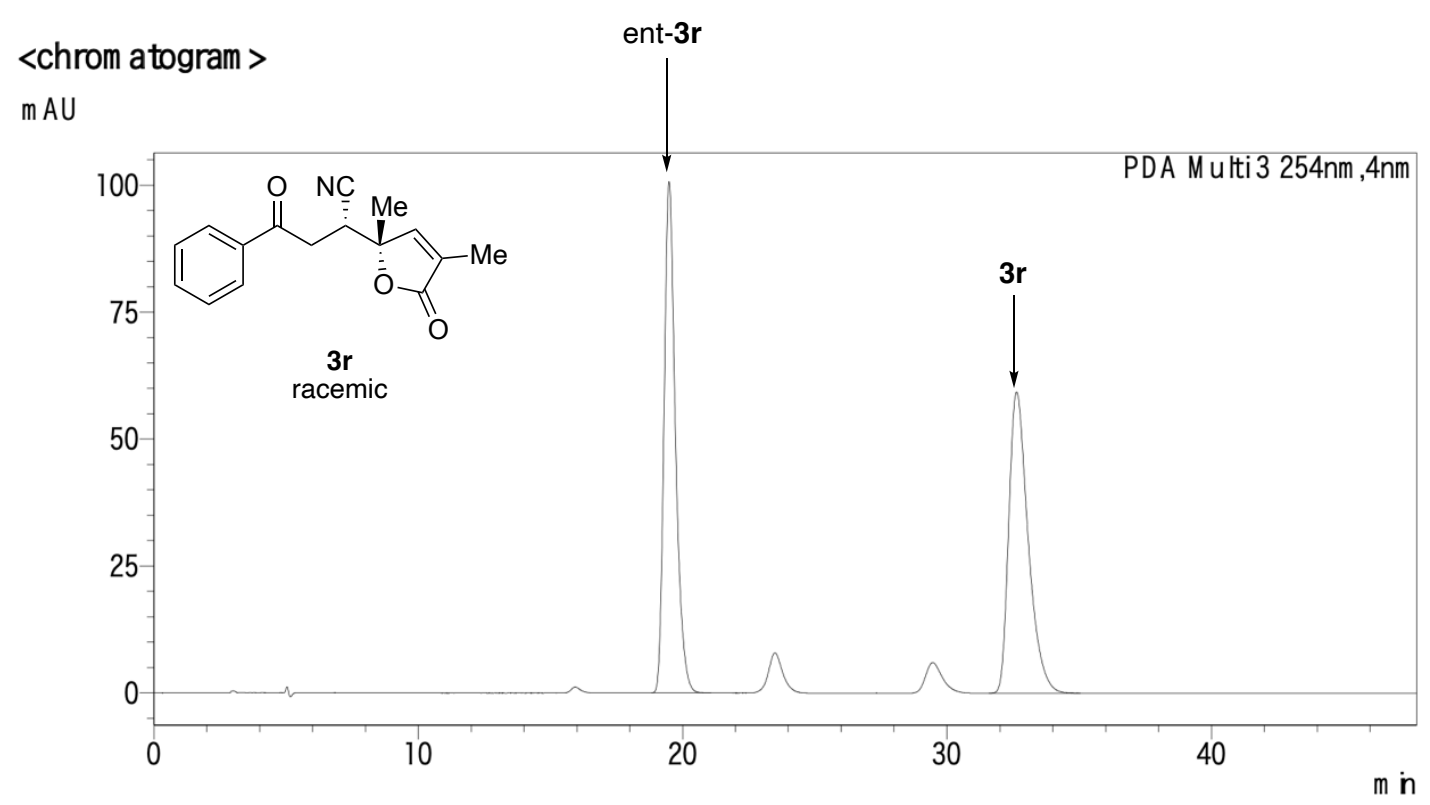

$<$ peak report

PDA Ch3 254nm

\begin{tabular}{|r|r|r|}
\hline peak\# & retention tim e & area\% \\
\hline 1 & 19.483 & \\
\hline 2 & 32.634 & 49.934 \\
\hline
\end{tabular}

$32.634 \quad 50.066$

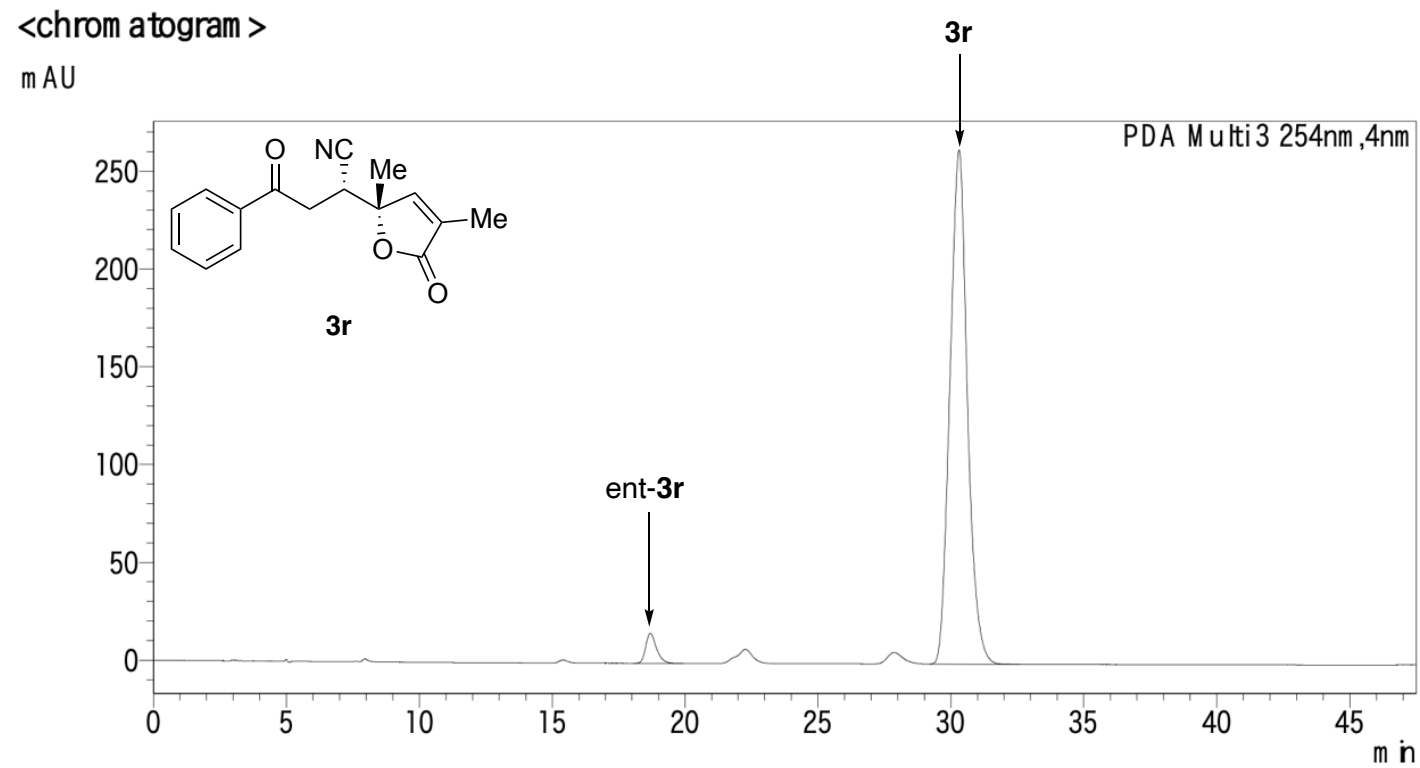

$<$ peak report

PDA Ch3 254nm

\begin{tabular}{|r|r|r|}
\hline peak\# & retention tim e & area\% \\
\hline 1 & 18.685 & 3.425 \\
\hline 2 & 30.311 & 96.575 \\
\hline
\end{tabular}




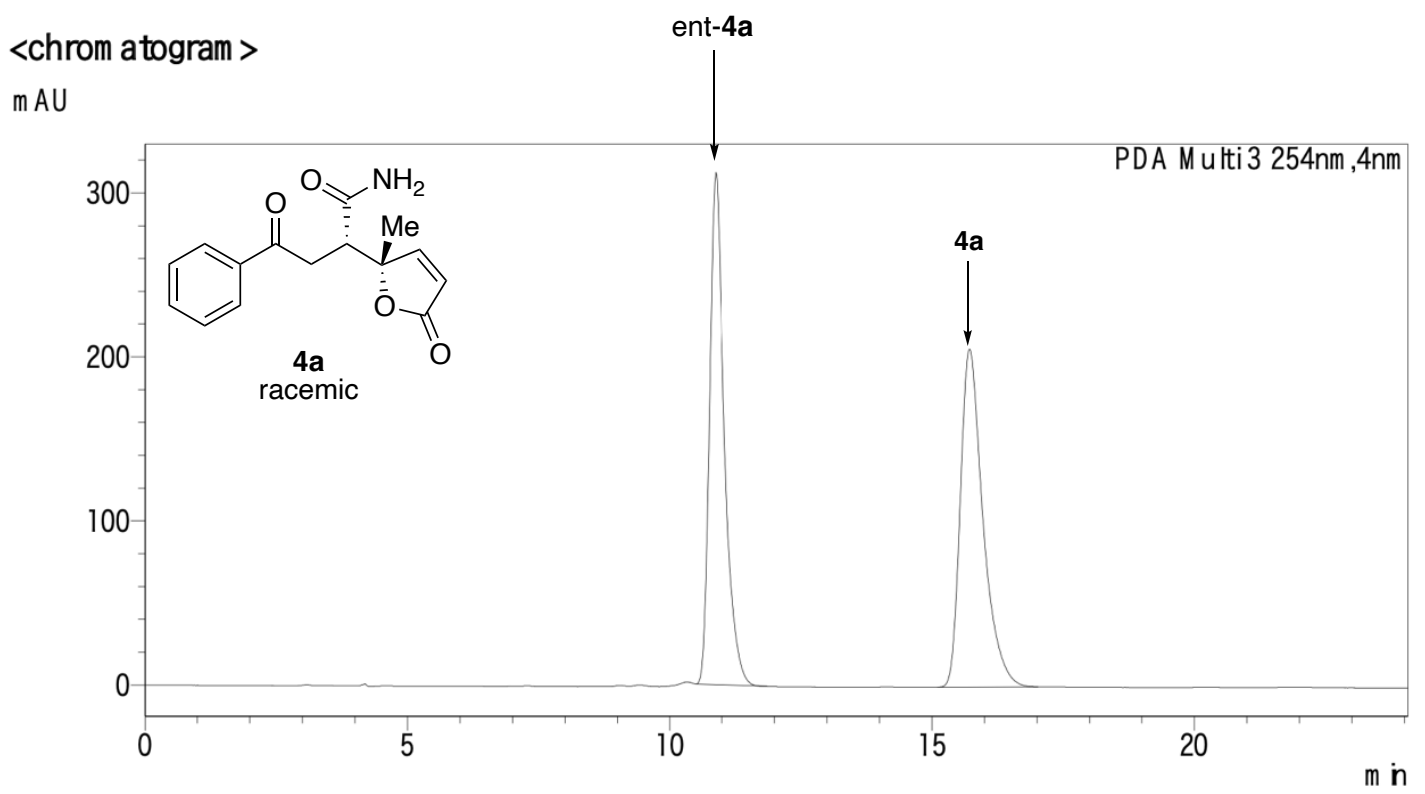

<peak report

PDA Ch3 254nm

\begin{tabular}{|r|r|r|}
\hline peak\# & retention tm e & \multicolumn{2}{c|}{ area\% } \\
\hline 1 & 10.880 & 49.805 \\
\hline 2 & 15.716 & 50.195 \\
\hline
\end{tabular}

<chrom atogram >

$\mathrm{mAU}$

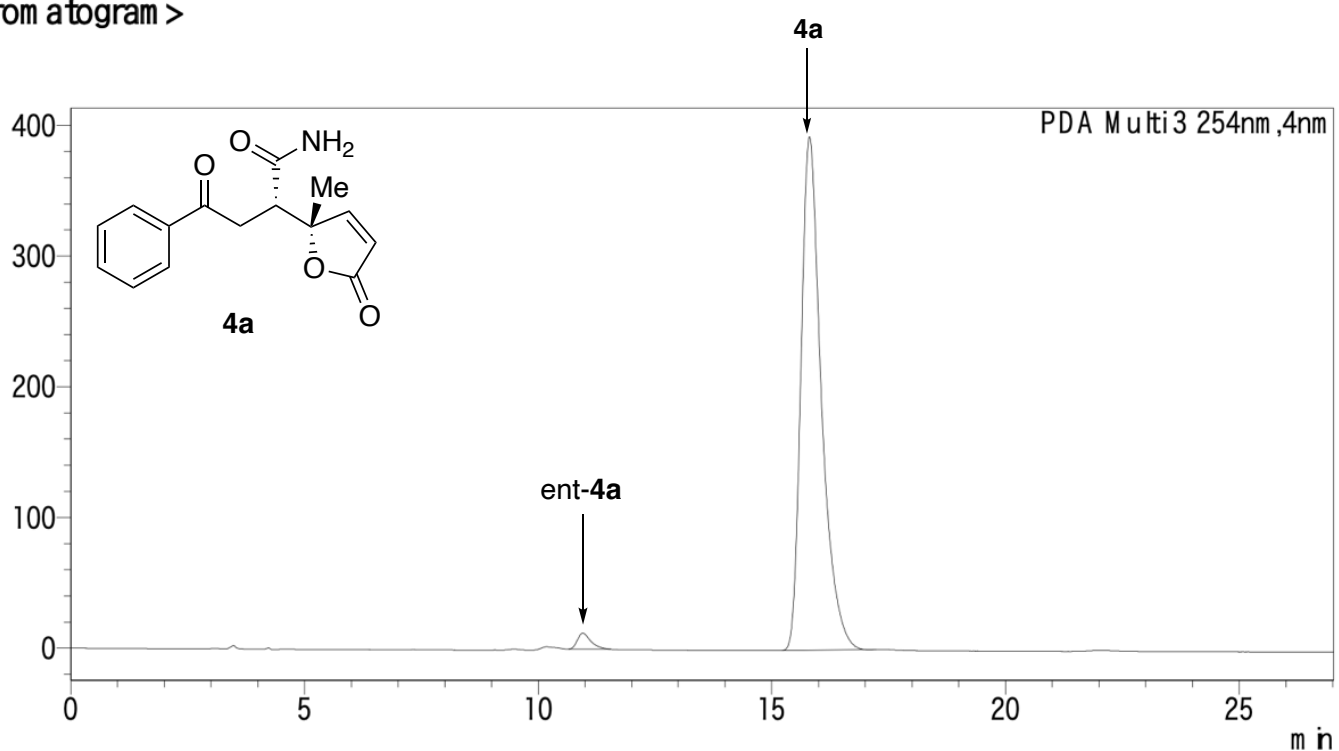

$<$ peak report

PDA Ch3 254nm

\begin{tabular}{|r|r|r|}
\hline peak\# & retention tim e & \multicolumn{2}{c|}{ area\% } \\
\hline 1 & 10.955 & 2.029 \\
\hline 2 & 15.812 & 97.971 \\
\hline
\end{tabular}




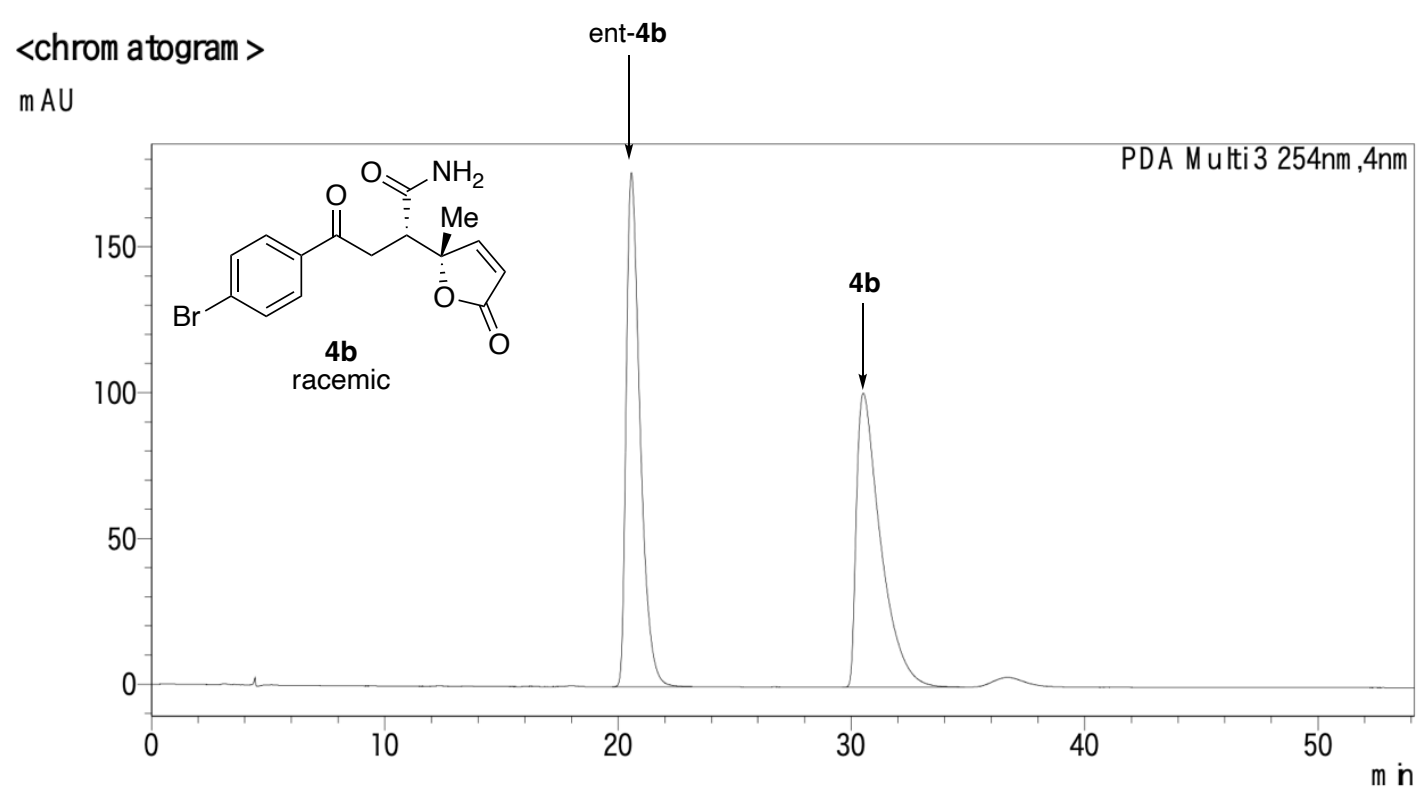

<peak report

PDA Ch3 254nm

\begin{tabular}{|r|r|r|} 
peak\# & retention time & \multicolumn{2}{c|}{ area\% } \\
\hline 1 & 20.566 & 50.021 \\
\hline 2 & 30.520 & 49.979 \\
\hline
\end{tabular}

$<$ chrom atogram >

$\mathrm{mAU}$

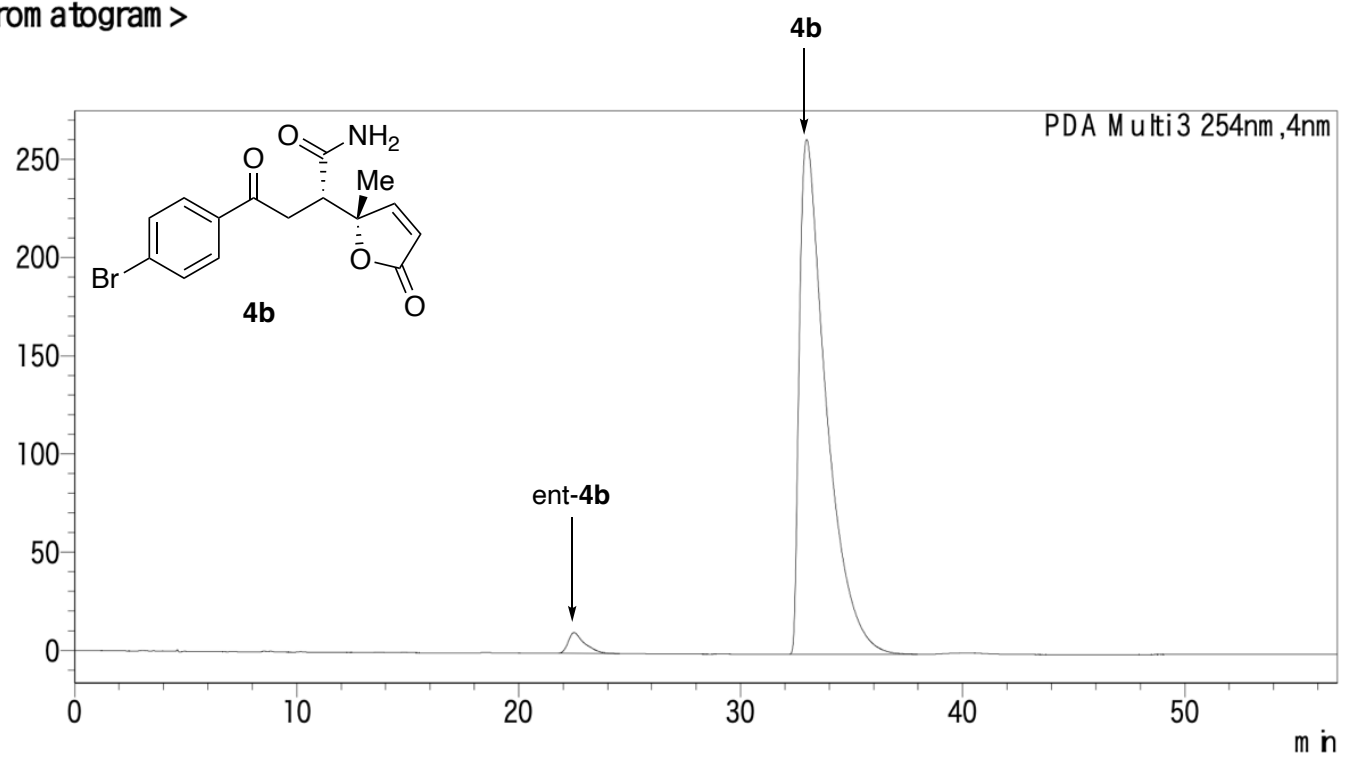

$<$ peak report

PDA Ch3 254nm

\begin{tabular}{|r|r|r|}
\hline peak\# & retention tim e & area\% \\
\hline 1 & 22.485 & 2.385 \\
\hline 2 & 32.977 & 97.615 \\
\hline
\end{tabular}




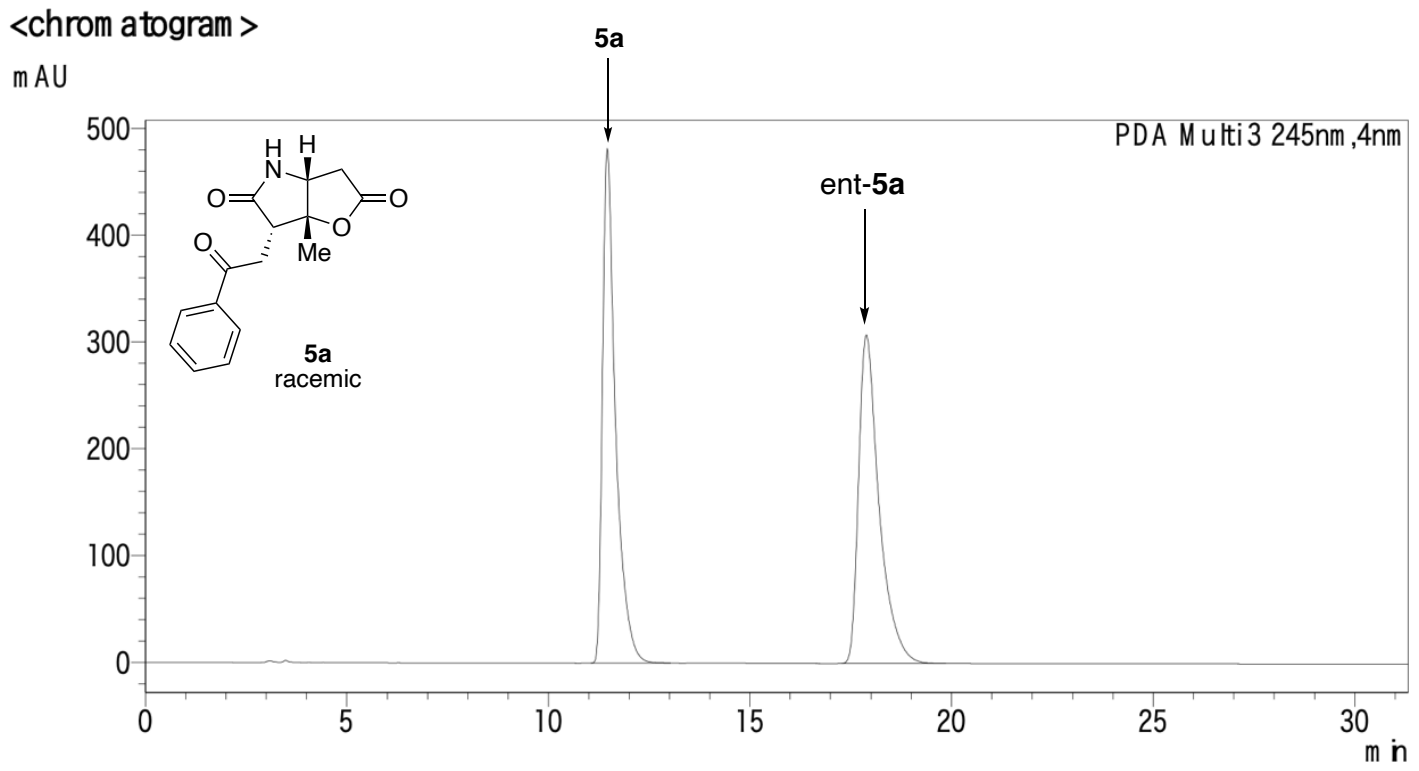

<peak report

PDA Ch3 245nm

\begin{tabular}{|r|r|r|}
\hline peak\# & retention tim e & \multicolumn{2}{c|}{ area\% } \\
\hline 1 & 11.457 & 49.942 \\
\hline 2 & 17.887 & 50.058 \\
\hline
\end{tabular}

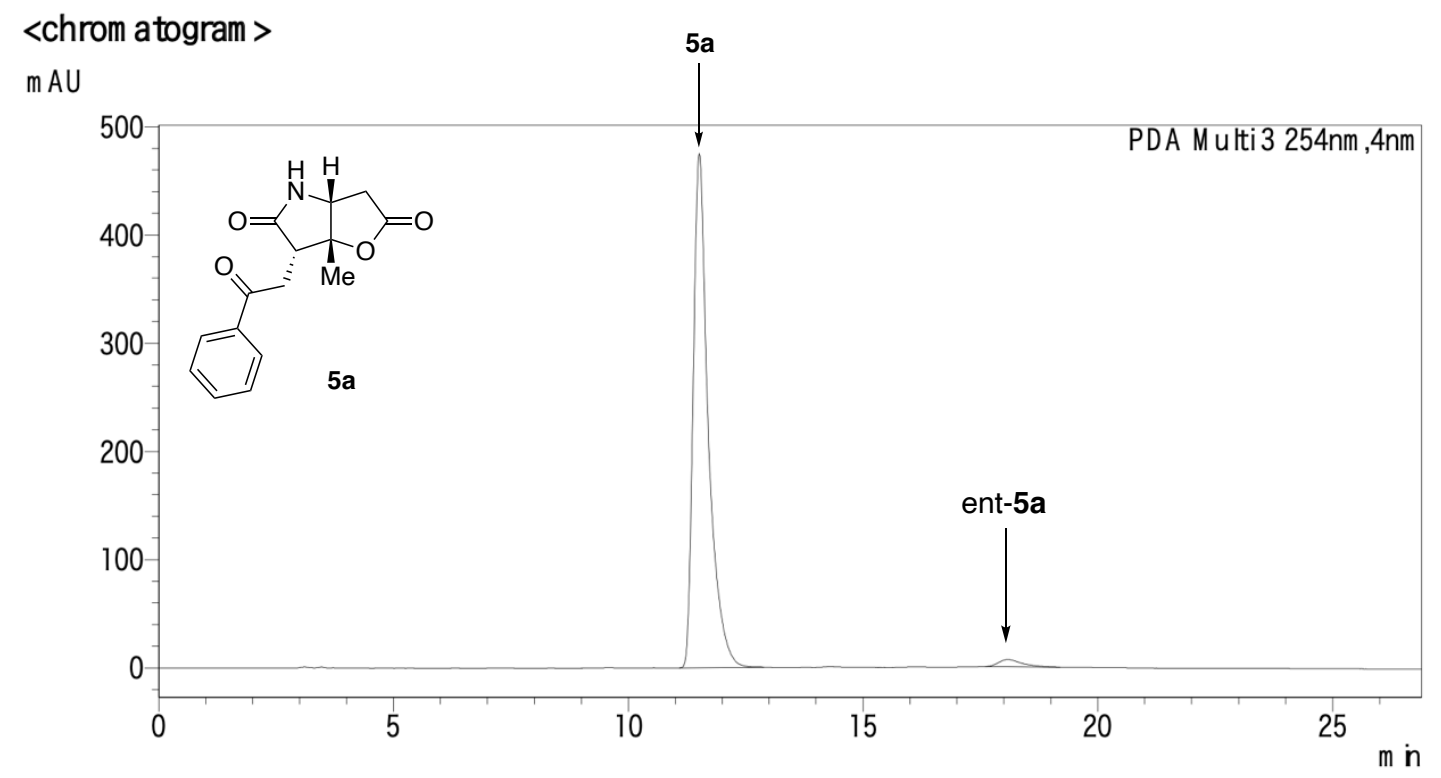

<peak report

PDA Ch3 $254 \mathrm{~nm}$

\begin{tabular}{|r|r|r|}
\hline peak\# & retention tim e & area\% \\
\hline 1 & 11.509 & 97.824 \\
\hline 2 & 18.081 & 2.176 \\
\hline
\end{tabular} 


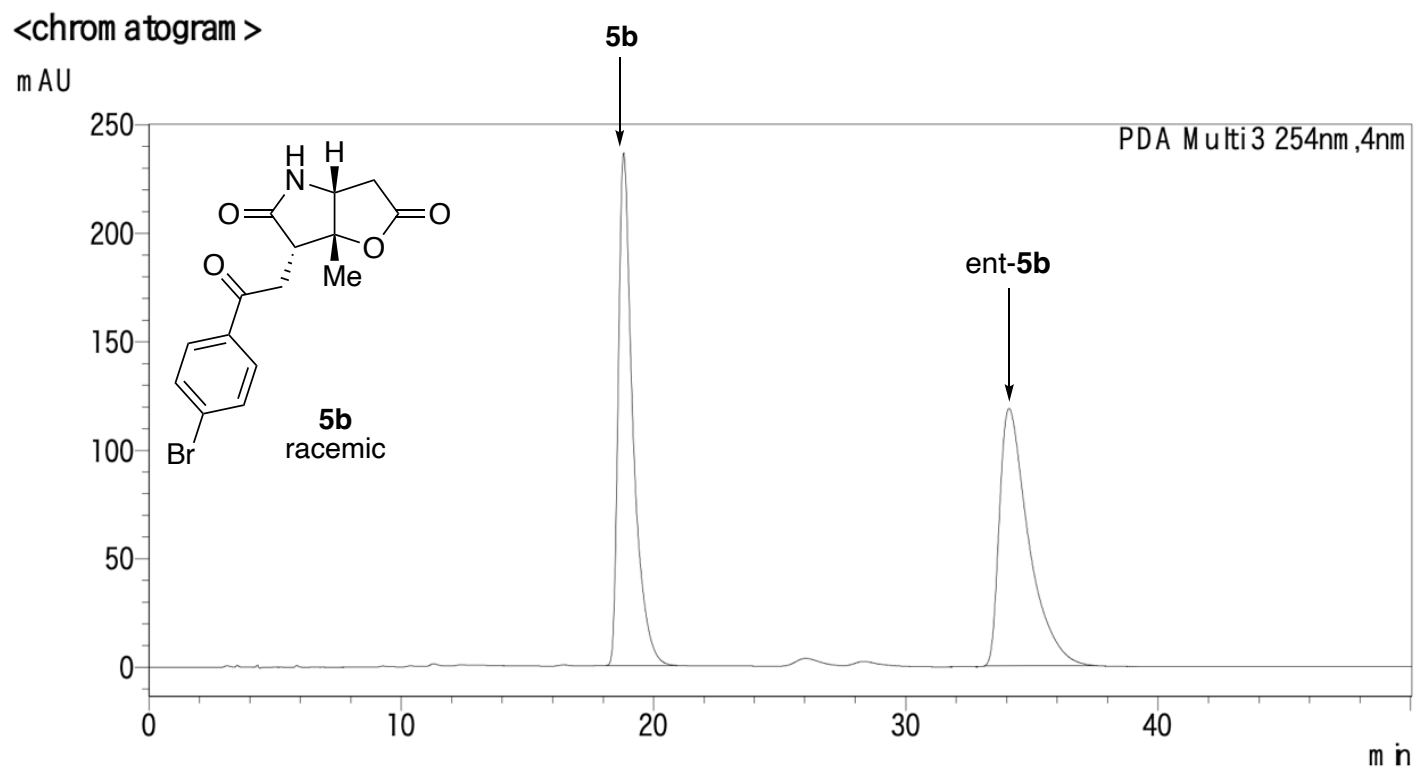

$<$ peak report

PDA Ch3 254nm

\begin{tabular}{|r|r|r|}
\hline peak\# & retention tim e & area\% \\
\hline 1 & 18.813 & 49.850 \\
\hline 2 & 34.092 & 50.150 \\
\hline
\end{tabular}

<chrom atogram >

$\mathrm{mAU}$

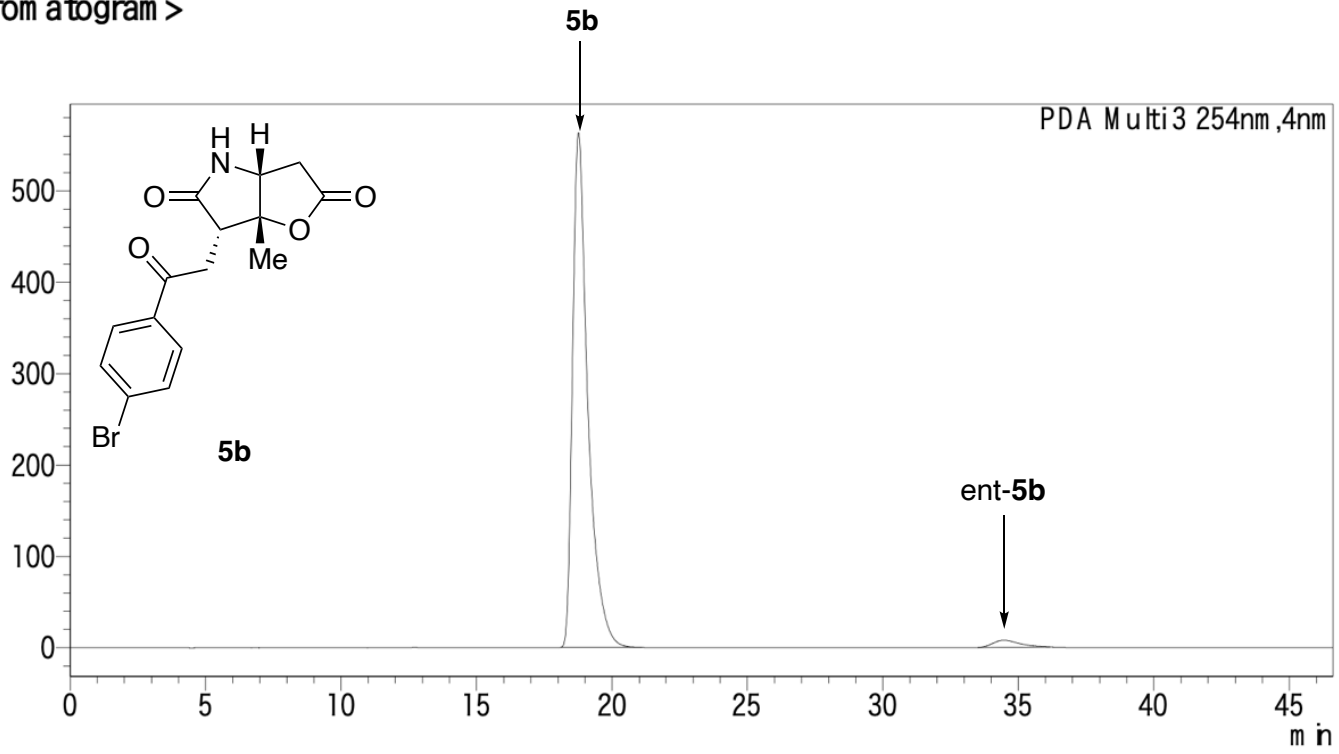

<peak report

PDA Ch3 254nm

\begin{tabular}{|r|r|r|}
\hline peak\# & retention tim e & area\% \\
\hline 1 & 18.758 & 97.648 \\
\hline 2 & 34.474 & 2.352 \\
\hline
\end{tabular}

